\title{
TRANSFORMED RANK-1 LATTICES FOR HIGH-DIMENSIONAL APPROXIMATION*
}

\author{
ROBERT NASDALA ${ }^{\dagger}$ AND DANIEL POTTS ${ }^{\dagger}$
}

\begin{abstract}
This paper describes an extension of Fourier approximation methods for multivariate functions defined on the torus $\mathbb{T}^{d}$ to functions in a weighted Hilbert space $L_{2}\left(\mathbb{R}^{d}, \omega\right)$ via a multivariate change of variables $\psi:\left(-\frac{1}{2}, \frac{1}{2}\right)^{d} \rightarrow \mathbb{R}^{d}$. We establish sufficient conditions for $\psi$ and $\omega$ such that the composition of a function in such a weighted Hilbert space with $\psi$ yields a function in the Sobolev space $H_{\text {mix }}^{m}\left(\mathbb{T}^{d}\right)$ of functions on the torus with mixed smoothness of natural order $m \in \mathbb{N}_{0}$. In this approach we adapt algorithms for the evaluation and reconstruction of multivariate trigonometric polynomials on the torus $\mathbb{T}^{d}$ based on single and multiple reconstructing rank- 1 lattices. Since in applications it may be difficult to choose a related function space, we make use of dimension incremental construction methods for sparse frequency sets. Various numerical tests confirm the obtained theoretical results for the transformed methods.
\end{abstract}

Key words. approximation on unbounded domains, change of variables, sparse multivariate trigonometric polynomials, lattice rule, multiple rank-1 lattice, fast Fourier transform

AMS subject classifications. 65T, 42B05

1. Introduction. The change of variables is a powerful tool in numerical analysis. Such transformations play an important role in spectral methods, numerical integration, and the approximation of functions. An excellent overview can be found in [1, Chapters 16 and 17], which contains many practical aspects of the mapping methods. In this paper we focus on change of variable mappings from multivariate bounded domains to unbounded ones in order to approximate functions defined on such unbounded domains. The main goal is to transfer the approximation error bounds of Fourier methods on the high-dimensional torus $\mathbb{T}^{d} \simeq\left[-\frac{1}{2}, \frac{1}{2}\right)^{d}$ to approximation methods on $\mathbb{R}^{d}$ with the help of an invertible transformation $\psi:\left(-\frac{1}{2}, \frac{1}{2}\right)^{d} \rightarrow \mathbb{R}^{d}$.

Regarding functions defined on the torus $\mathbb{T}^{d}$, there is a well-developed theory (see $[7,20,27])$ concerned with the Wiener algebra $\mathcal{A}\left(\mathbb{T}^{d}\right)$ that contains all $L_{1}\left(\mathbb{T}^{d}\right)$-functions with absolutely summable Fourier coefficients

$$
\hat{f}_{\mathbf{k}}:=\int_{\mathbb{T}^{d}} f(\mathbf{x}) \mathrm{e}^{-2 \pi \mathrm{i} \mathbf{k} \cdot \mathbf{x}} \mathrm{d} \mathbf{x}
$$

with $\mathbf{k}=\left(k_{1}, \ldots, k_{d}\right)^{\top} \in \mathbb{Z}^{d}, \mathbf{x}=\left(x_{1}, \ldots, x_{d}\right)^{\top} \in \mathbb{R}^{d}$, and $\mathbf{k} \cdot \mathbf{x}:=\sum_{j=1}^{d} k_{j} x_{j}$. For $\beta \geq 0$ and the weight function

$$
\omega_{\mathrm{hc}}(\mathbf{k}):=\prod_{j=1}^{d} \max \left(1,\left|k_{j}\right|\right)
$$

there are subspaces of the Wiener algebra $\mathcal{A}\left(\mathbb{T}^{d}\right)$ in form of

$$
\mathcal{A}^{\beta}\left(\mathbb{T}^{d}\right):=\left\{f \in L_{1}\left(\mathbb{T}^{d}\right):\|f\|_{\mathcal{A}^{\beta}\left(\mathbb{T}^{d}\right)}:=\sum_{\mathbf{k} \in \mathbb{Z}^{d}} \omega_{\mathrm{hc}}(\mathbf{k})^{\beta}\left|\hat{f}_{\mathbf{k}}\right|<\infty\right\}
$$

*Received September 2, 2019. Accepted December 18, 2019. Published online on February 5, 2020. Recommended by L. Reichel.

${ }^{\dagger}$ Technische Universität Chemnitz, Faculty of Mathematics, 09107 Chemnitz, Germany

( $\{$ robert.nasdala, daniel.potts $\}$ @math.tu-chemnitz.de). 
and the Hilbert spaces

$$
\mathcal{H}^{\beta}\left(\mathbb{T}^{d}\right):=\left\{f \in L_{2}\left(\mathbb{T}^{d}\right):\|f\|_{\mathcal{H}^{\beta}\left(\mathbb{T}^{d}\right)}:=\left(\sum_{\mathbf{k} \in \mathbb{Z}^{d}} \omega_{\mathrm{hc}}(\mathbf{k})^{2 \beta}\left|\hat{f}_{\mathbf{k}}\right|^{2}\right)^{\frac{1}{2}}<\infty\right\},
$$

whose norms contain information about the decay rate of the Fourier coefficients $\hat{f}_{\mathbf{k}}$ with respect to the weight function $\omega_{\mathrm{hc}}$. For approximation purposes we consider non-empty frequency sets $I \subset \mathbb{Z}^{d}$ of finite cardinality $|I|<\infty$ and approximated Fourier partial sums

$$
S_{I}^{\Lambda} f(\mathbf{x}):=\sum_{\mathbf{k} \in I} \hat{f}_{\mathbf{k}}^{\Lambda} \mathrm{e}^{2 \pi \mathbf{i k} \cdot \mathbf{x}}
$$

with approximated Fourier coefficients

$$
\hat{f}_{\mathbf{k}}^{\Lambda}:=\frac{1}{M} \sum_{j=0}^{M-1} f\left(\mathbf{x}_{j}\right) \mathrm{e}^{-2 \pi \mathrm{i} \mathbf{k} \cdot \mathbf{x}_{j}} \approx \hat{f}_{\mathbf{k}}
$$

which are sampled at the nodes $\mathbf{x}_{j}$ of a reconstructing rank-1 lattice $\Lambda(\mathbf{z}, M, I)$, whose definition is given in (2.6).

For $N \in \mathbb{N}$ and hyperbolic crosses

$$
I_{N}^{d}:=\left\{\mathbf{k} \in \mathbb{Z}^{d}: \omega_{\mathrm{hc}}(\mathbf{k}) \leq N\right\},
$$

it was shown in [13, Theorem 3.3] that when using single rank-1 lattices, the error of approximating a continuous function $f \in \mathcal{A}^{\beta}\left(\mathbb{T}^{d}\right)$ by the approximated Fourier partial sum $S_{I_{N}^{d}}^{\Lambda} f$ measured in the $L_{\infty}\left(\mathbb{T}^{d}\right)$-norm is bounded above by $N^{-\beta}\|f\|_{\mathcal{A}^{\beta}\left(\mathbb{T}^{d}\right)}$. The approximation of functions in the Hilbert spaces $\mathcal{H}^{\beta}\left(\mathbb{T}^{d}\right)$ was investigated by V. N. Temlyakov in, e.g., [13, 26]. For certain $\beta \geq 0$, the error of approximating a continuous function $f \in \mathcal{H}^{\beta}\left(\mathbb{T}^{d}\right)$ by the approximated Fourier partial sum $S_{I_{N}^{d}}^{\Lambda} f$ measured in the $L_{2}\left(\mathbb{T}^{d}\right)$-norm is bounded above by $C_{d, \beta} N^{-\beta}(\log N)^{(d-1) / 2}\|f\|_{\mathcal{H}^{\beta}\left(\mathbb{T}^{d}\right)}$ with some constant $C_{d, \beta}=C(d, \beta)>0$ as shown in [30, Theorem 2.30].

A major problem is that in general it is difficult to calculate the Fourier coefficients $\hat{f}_{\mathbf{k}}$ in order to determine if they are absolutely or square summable. Instead we utilize certain norm equivalences to get information about the decay rate of the Fourier coefficients $\hat{f}_{\mathbf{k}}$. Given a multi-index $\boldsymbol{\alpha}=\left(\alpha_{1}, \ldots, \alpha_{d}\right)^{\top} \in \mathbb{N}_{0}^{d}$ with $\|\boldsymbol{\alpha}\|_{\ell_{\infty}}:=\max \left(\left|\alpha_{1}\right|, \ldots,\left|\alpha_{d}\right|\right)$ and the differential operator

$$
D^{\boldsymbol{\alpha}}[f](\mathbf{x})=D^{\left(\alpha_{1}, \ldots, \alpha_{d}\right)}[f]\left(x_{1}, \ldots, x_{d}\right):=\frac{\partial^{\alpha_{1}}}{\partial x_{1}^{\alpha_{1}}} \ldots \frac{\partial^{\alpha_{d}}}{\partial x_{d}^{\alpha_{d}}}[f]\left(x_{1}, \ldots, x_{d}\right),
$$

we define for $\Omega \in\left\{\mathbb{T}^{d}, \mathbb{R}^{d}\right\}$ the norm

$$
\|f\|_{H_{\text {mix }}^{m}(\Omega)}:=\left(\sum_{\|\boldsymbol{\alpha}\|_{\ell_{\infty}} \leq m}\left\|D^{\boldsymbol{\alpha}}[f]\right\|_{L_{2}(\Omega)}^{2}\right)^{1 / 2}
$$

of the Sobolev space $H_{\text {mix }}^{m}(\Omega)$ of functions $f \in L_{2}(\Omega)$ with mixed natural smoothness $m \in \mathbb{N}_{0}$, which is discussed in [22, 28, 31]. As shown in [17, Lemma 2.3], the norms $\|\cdot\|_{H_{\text {mix }}^{m}\left(\mathbb{T}^{d}\right)}$ and $\|\cdot\|_{\mathcal{H}^{\beta}\left(\mathbb{T}^{d}\right)}$ are equivalent for $\beta=m \in \mathbb{N}$. Furthermore, for all $\beta \geq 0$ and all $\lambda>\frac{1}{2}$, we have 
the continuous embedding $\mathcal{H}^{\beta+\lambda}\left(\mathbb{T}^{d}\right) \hookrightarrow \mathcal{A}^{\beta}\left(\mathbb{T}^{d}\right)$ as shown in [13, Lemma 2.2]. Hence, for $m \in \mathbb{N}$, we can simply verify that $f$ is an element of a Sobolev space $H_{\text {mix }}^{m}\left(\mathbb{T}^{d}\right)$ in order to determine whether a function $f$ is in $\mathcal{A}^{m}\left(\mathbb{T}^{d}\right)$ or $\mathcal{H}^{m}\left(\mathbb{T}^{d}\right)$ instead of calculating all its Fourier coefficients $\hat{f}_{\mathbf{k}}$.

In order to utilize all these properties for functions defined on $\mathbb{R}^{d}$, we apply a continuously differentiable and strictly increasing change of variables $\psi:\left(-\frac{1}{2}, \frac{1}{2}\right)^{d} \rightarrow \mathbb{R}^{d}$ component-wise to multivariate functions $h$ in a weighted Hilbert space $L_{2}\left(\mathbb{R}^{d}, \omega\right)$ as defined in (2.1) with the weight function $\omega: \mathbb{R}^{d} \rightarrow[0, \infty)$. As a result we consider transformed functions $f \in L_{2}\left(\mathbb{T}^{d}\right)$ of the form

$$
f(\mathbf{x})=h(\psi(\mathbf{x})) \sqrt{\omega(\psi(\mathbf{x})) \psi^{\prime}(\mathbf{x})},
$$

so that we have the identity $\|h\|_{L_{2}\left(\mathbb{R}^{d}, \omega\right)}=\|f\|_{L_{2}\left(\mathbb{T}^{d}\right)}$. Based on this connection we will later on observe that the inverse transformation $\psi^{-1}$ transforms the classical Fourier system $\left\{\mathrm{e}^{2 \pi \mathrm{ik} \circ}\right\}$ into another orthonormal system of the form $\left\{\sqrt{\frac{\left(\psi^{-1}\right)^{\prime}(\circ)}{\omega(0)}} \mathrm{e}^{2 \pi \mathbf{i} \cdot \psi^{-1}(\circ)}\right\}$. It is generally rather difficult to verify whether such a transformed function $f$ is in the Sobolev space $H_{\text {mix }}^{m}\left(\mathbb{T}^{d}\right)$ by calculating its norm and testing the various $L_{2}$-integrability conditions. Therefore we provide a set of sufficient $L_{\infty}$-conditions for $f$ being in $H_{\text {mix }}^{m}\left(\mathbb{T}^{d}\right)$.

At first we prove these conditions for all possible transformations $\psi$ and weight functions $\omega$. Later on, we consider families of parameterized transformations $\psi(\circ)=\psi(\circ, \boldsymbol{\eta})$ and families of weight functions $\omega(\circ)=\omega(\circ, \boldsymbol{\mu})$ with $\boldsymbol{\eta}, \boldsymbol{\mu} \in \mathbb{R}^{d}$. Then we obtain parameterized transformed functions $f(\circ)=f(\circ, \boldsymbol{\eta}, \boldsymbol{\mu}) \in L_{2}\left(\mathbb{T}^{d}\right)$, and both parameters may influence the smoothness of these functions. With the sufficient $L_{\infty}$-smoothness conditions, we are then able to calculate lower bounds for $\boldsymbol{\eta}$ and $\boldsymbol{\mu}$ such that the smoothness degree $m$ of a function $h \in L_{2}\left(\mathbb{R}^{d}, \omega(\circ, \boldsymbol{\mu})\right) \cap H_{\text {mix }}^{m}\left(\mathbb{R}^{d}\right)$ does not change under composition with a family of transformations $\psi(\circ, \boldsymbol{\eta})$ so that we end up with $f \in H_{\text {mix }}^{m}\left(\mathbb{T}^{d}\right)$.

For two particular transformation families $\psi(\circ, \boldsymbol{\eta})$ we explicitly calculate the resulting lower parameter bounds and observe a case in which the smoothness preservation under the transformation depends only on the parameter $\boldsymbol{\mu} \in \mathbb{R}^{d}$ appearing in the weight functions $\omega(\circ, \boldsymbol{\mu})$, as far as the conditions are able to detect it. Furthermore, we present an example in which we compare the parameter bounds obtained from the $L_{\infty}$-conditions with the exact lower bounds resulting from calculating the Sobolev-norm $\|\cdot\|_{H_{\text {mix }}^{m}\left(\mathbb{T}^{d}\right)}$. This will highlight that the easier to verify $L_{\infty}$-conditions yield slightly coarser parameter bounds. These conditions, as a tool to determine when a transformed function $f$ is at least an $L_{2}\left(\mathbb{T}^{d}\right)$-function, enable us to prove upper bounds for the approximation error $\left\|h-S_{I}^{\Lambda} h\right\|$ measured in the weighted $L_{2}$ and $L_{\infty}$-norms on $\mathbb{R}^{d}$. These are based on the already established error bounds for $\left\|f-S_{I}^{\Lambda} f\right\|$ on the torus with respect to the $L_{2}\left(\mathbb{T}^{d}\right)$ - and $L_{\infty}\left(\mathbb{T}^{d}\right)$-norms.

One advantage of the proposed method is the availability of fast algorithms for highdimensional approximation (see, e.g., [1]) in contrast to function approximations based on, for instance, multivariate Hermite functions or Sinc methods. To this end, there are lattice rules that in recent years became an important tool in numerical analysis for high-dimensional integration and the approximation of multivariate functions. An introduction to lattice rules can be found in $[6,19,24]$. These rules are used for the approximation of functions on the torus; see [27]. Recently, efficient algorithms based on component-by-component methods [4, 5] were presented in order to compute high-dimensional integrals. For the approximation of high-dimensional functions, there are efficient algorithms using sampling schemes based on rank-1 lattices [8, 13], and furthermore, these schemes provide good approximation properties; see also [2]. We adapt these algorithms and incorporate the outlined use of transformations. Furthermore, we present numerical examples. 
We note that it was recently suggested in $[11,12]$ to use multiple rank-1 lattices which are obtained by taking a union of several single rank- 1 lattices. This method overcomes the limitations of the single rank- 1 lattice approach. That is, for the reconstruction of multivariate trigonometric polynomials supported on an arbitrary frequency set $I$ of finite cardinality $|I|<\infty$, with a single reconstructing rank-1 lattice, the lattice size $M$ is bounded by $|I| \leq M \leq|I|^{2}$ under certain mild assumptions; see [13, Lemma 2.1] and [10, Corollary 1]. Multiple rank-1 lattices improve the upper bound to $M \leq C|I| \log |I|$ with high probability $[12,14]$. Remarkably, in both cases the upper bound is independent of the dimension $d$. Furthermore, there are methods where the support of the Fourier coefficients $\hat{f}_{\mathbf{k}}$ is unknown. We adapt the methods presented in [21] that describe a dimension incremental construction of a frequency set $I \subset \mathbb{Z}^{d}$ containing only non-zero or the approximately largest Fourier coefficients $\hat{h}_{\mathbf{k}}$, based on a component-by-component construction of rank-1 lattices. This is done with respect to a specific search space in form of a full integer grid $[-N, N]^{d} \cap \mathbb{Z}^{d}$ with refinement $N \in \mathbb{N}$ and a sparsity constraint that bounds the cardinality of the support. We incorporate the change of variables method into both the multiple rank-1 lattice methods as well as the component-by-component construction method. Let us note that instead of rank-1 lattice points, one can use a dimensional incremental support identification technique based on randomly chosen sampling points, which was recently developed in [3].

The outline of the paper is as follows: In Section 2 we establish the basic notions from classical Fourier approximation theory on the torus $\mathbb{T}^{d}$, the corresponding function spaces, and important convergence properties. We introduce the Sobolev spaces $H_{\text {mix }}^{m}\left(\mathbb{T}^{d}\right)$ of mixed natural smoothness order $m \in \mathbb{N}_{0}$ and the Wiener Algebra $\mathcal{A}\left(\mathbb{T}^{d}\right)$ of functions with absolutely summable Fourier coefficients. Furthermore, we discuss certain properties of the subspaces $\mathcal{A}^{\beta}\left(\mathbb{T}^{d}\right)$ and $\mathcal{H}^{\beta}\left(\mathbb{T}^{d}\right)$ of the Wiener Algebra, in particular, we highlight the norm equivalence of $\|\cdot\|_{\mathcal{H}^{m}\left(\mathbb{T}^{d}\right)}$ and $\|\cdot\|_{H_{\text {mix }}^{m}\left(\mathbb{T}^{d}\right)}$ for all $m \in \mathbb{N}$; see [17]. Then we define rank-1 lattices as introduced in [15], discuss their importance in the context of Fourier approximation, and recall two important approximation error bounds on the torus in Theorems 2.2 and 2.3.

In Section 3 we define the notion of a transformation $\psi:\left(-\frac{1}{2}, \frac{1}{2}\right)^{d} \rightarrow \mathbb{R}^{d}$ and provide a couple of examples that we will use later on. Then we introduce weight functions $\omega: \mathbb{R}^{d} \rightarrow[0, \infty)$ and describe the structure of the weighted Hilbert spaces $L_{2}\left(\mathbb{R}^{d}, \omega\right)$, the corresponding weighted scalar product $(\cdot, \cdot)_{L_{2}\left(\mathbb{R}^{d}, \omega\right)}$, and the resulting Fourier coefficients $\hat{h}_{\mathbf{k}}$. Afterwards, we prove sufficient $L_{\infty}$-conditions for the transformation $\psi$ and the weight function $\omega$ such that a function $h \in L_{2}\left(\mathbb{R}^{d}, \omega\right) \cap H_{\text {mix }}^{m}\left(\mathbb{R}^{d}\right)$ is transformed under composition with $\psi$ into a smooth function $f \in H_{\text {mix }}^{m}\left(\mathbb{T}^{d}\right)$. Then we are able to prove approximation error bounds on $\mathbb{R}^{d}$ in Theorems 3.6 and 3.7 based on the theorems on the torus from Section 2.

In Section 4, we incorporate the usage of transformations $\psi$ into the algorithms [8, Algorithm 3.1 and 3.2] for the evaluation and the reconstruction of multivariate functions leading to Algorithms 4.1 and 4.2 based on transformed rank-1 lattices.

In Section 5 we discuss examples for the algebraic transformation (3.6) and the error function transformation (3.8) that were introduced in Section 3. In these examples we use a parameterized transformation $\psi(\circ)=\psi(\circ, \boldsymbol{\eta})$ with $\boldsymbol{\eta} \in \mathbb{R}^{d}$ and a parameterized weight function $\omega(\circ)=\omega(\circ, \boldsymbol{\mu})$ with $\boldsymbol{\mu} \in \mathbb{R}^{d}$ that fit with their original definitions in Sections 2 and 3. With the sufficient $L_{\infty}$-conditions from Section 3, we then calculate explicit lower bounds for $\boldsymbol{\eta}$ and $\boldsymbol{\mu}$ determining the degree of smoothness $m \in \mathbb{N}$ of $h \in L_{2}\left(\mathbb{R}^{d}, \omega(\circ, \boldsymbol{\mu})\right) \cap H_{\text {mix }}^{m}\left(\mathbb{R}^{d}\right)$, which is preserved under composition with the family of transformations $\psi(\circ)=\psi(\circ, \boldsymbol{\eta})$. Then we use the algorithms of the previous section to illustrate the theoretical upper approximation error bounds. For some special cases in which the Fourier coefficients $\hat{h}_{\mathbf{k}}$ are explicitly given, we compare those to the theoretically predicted rate of decay of their absolutely values. 
In Section 6 we add some remarks on how the tool of change of variables is incorporated into the ideas of multiple rank-1 lattices and sparse fast Fourier algorithms. Furthermore, we present examples with various test functions and different transformation maps in up to $d=12$ dimensions.

2. Fourier approximation on the torus. At first we introduce weighted $L_{p}$-function spaces and Sobolev spaces of mixed smoothness, recall some definitions of classical Fourier approximation theory, and define a space of functions with absolute square-summable Fourier coefficients. Finally, we review the ideas of rank-1 lattices from [4, 8, 25], the corresponding Fourier approximation methods, and approximation error bounds that were discussed in, e.g., $[2,13,26]$.

2.1. Preliminaries. Let $\Omega \in\left\{\mathbb{T}^{d}, \mathbb{R}^{d}\right\}$, with $\mathbb{T}^{d} \simeq\left[-\frac{1}{2}, \frac{1}{2}\right)^{d}$ being the $d$-dimensional torus. The space $\left(\mathcal{C}(\Omega),\|\cdot\|_{L_{\infty}(\Omega)}\right)$ denotes the collection of all continuous functions $f: \Omega \rightarrow \mathbb{C}$, and $\left(\mathcal{C}_{0}\left(\mathbb{R}^{d}\right),\|\cdot\|_{L_{\infty}\left(\mathbb{R}^{d}\right)}\right)$ denotes the space of all continuous functions $f: \mathbb{R}^{d} \rightarrow \mathbb{C}$ vanishing at infinity in every direction. We define weighted function spaces $L_{p}\left(\mathbb{R}^{d}, \omega\right)$ for $1 \leq p<\infty$ with the weight function $\omega: \mathbb{R}^{d} \rightarrow[0, \infty)$ as

$$
L_{p}\left(\mathbb{R}^{d}, \omega\right):=\left\{h \in L_{p}\left(\mathbb{R}^{d}\right):\|h\|_{L_{p}\left(\mathbb{R}^{d}, \omega\right)}:=\left(\int_{\mathbb{R}^{d}}|h(\mathbf{x})|^{p} \omega(\mathbf{x}) \mathrm{d} \mathbf{x}\right)^{\frac{1}{p}}<\infty\right\}
$$

with the usual adjustments for $p=\infty$. We have $L_{p}\left(\mathbb{R}^{d}\right) \subset L_{p}\left(\mathbb{R}^{d}, \omega\right)$ if $\omega$ is bounded and $L_{p}\left(\mathbb{R}^{d}, \omega\right) \subset L_{p}\left(\mathbb{R}^{d}\right)$ if $\omega$ is unbounded. For the constant weight function $\omega(\mathbf{x}) \equiv 1$, we have $L_{p}\left(\mathbb{R}^{d}, \omega\right)=L_{p}\left(\mathbb{R}^{d}\right)$, and the $L_{p}\left(\mathbb{T}^{d}\right)$-spaces are defined analogously.

For functions $f$ and $g$ in the Hilbert space $L_{2}\left(\mathbb{T}^{d}\right)$, we have the scalar product

$$
(f, g)_{L_{2}\left(\mathbb{T}^{d}\right)}:=\int_{\mathbb{T}^{d}} f(\mathbf{x}) \overline{g(\mathbf{x})} \mathrm{d} \mathbf{x} .
$$

The functions $\mathrm{e}^{2 \pi \mathrm{ik} \cdot \mathbf{x}}:=\prod_{j=1}^{d} \mathrm{e}^{2 \pi \mathrm{i} k_{j} x_{j}}$ with $\mathbf{k} \in \mathbb{Z}^{d}$ and $\mathbf{x} \in \mathbb{T}^{d}$ are orthogonal with respect to the $L_{2}\left(\mathbb{T}^{d}\right)$-scalar product. For any frequency set $I \subset \mathbb{Z}^{d}$ of finite cardinality $|I|<\infty$, we denote by

$$
\Pi_{I}:=\operatorname{span}\left\{\mathrm{e}^{2 \pi \mathrm{ik} \cdot o}: \mathbf{k} \in I\right\}
$$

the space of all multivariate trigonometric polynomials supported on $I$. For all $\mathbf{k} \in \mathbb{Z}^{d}$ we denote the Fourier coefficients $\hat{f}_{\mathbf{k}}$ by

$$
\hat{f}_{\mathbf{k}}=\left(f, \mathrm{e}^{2 \pi \mathrm{i} \mathbf{k} \cdot 0}\right)_{L_{2}\left(\mathbb{T}^{d}\right)}=\int_{\mathbb{T}^{d}} f(\mathbf{x}) \mathrm{e}^{-2 \pi \mathrm{i} \mathbf{k} \cdot \mathbf{x}} \mathrm{d} \mathbf{x}
$$

and the corresponding Fourier partial sum by $S_{I} f(\mathbf{x}):=\sum_{\mathbf{k} \in I} \hat{f}_{\mathbf{k}} \mathrm{e}^{2 \pi \mathrm{ik} \cdot \mathbf{x}}$.

For multi-indices $\boldsymbol{\alpha} \in \mathbb{N}_{0}^{d}$ and the differential operator $D^{\boldsymbol{\alpha}}[f](\mathbf{x})$ as defined in (1.6), we define the Sobolev spaces of mixed natural smoothness of $L_{2}(\Omega)$-functions with smoothness order $m \in \mathbb{N}_{0}$ (see $[22,28,31]$ ) as

$$
H_{\text {mix }}^{m}(\Omega)=\left\{f \in L_{2}(\Omega):\|f\|_{H_{\text {mix }}^{m}(\Omega)}<\infty\right\}
$$

with $\|\cdot\|_{H_{\text {mix }}^{m}(\Omega)}$ as given in (1.7). The univariate spaces are denoted by $H^{m}(\mathbb{T})$ and $H^{m}(\mathbb{R})$, respectively. For $\Omega=\mathbb{T}^{d}$ we recall some notation introduced in [17]. The $H_{\mathrm{mix}}^{m}\left(\mathbb{T}^{d}\right)$-norm is 
expressible in terms of the Fourier coefficients $\hat{f}_{\mathbf{k}}$, which leads to the equivalent norm

$$
\|f\|_{H^{m,+}\left(\mathbb{T}^{d}\right)}:=\left(\sum_{\mathbf{k} \in \mathbb{Z}^{d}}\left|\hat{f}_{\mathbf{k}}\right|^{2} \prod_{j=1}^{d}\left(1+\left|k_{j}\right|^{2}\right)^{m}\right)^{\frac{1}{2}} .
$$

In [17, Lemma 2.3] it is specified that for $m \in \mathbb{N}$ and all $f \in H_{\text {mix }}^{m}\left(\mathbb{T}^{d}\right)$, we have

$$
\|f\|_{H_{\text {mix }}^{m}\left(\mathbb{T}^{d}\right)} \leq\|f\|_{H^{m,+}\left(\mathbb{T}^{d}\right)} \leq\left(\frac{2^{m}}{m+1}\right)^{\frac{d}{2}}\|f\|_{H_{\text {mix }}^{m}\left(\mathbb{T}^{d}\right)} .
$$

The $\|\cdot\|_{H^{m,+}\left(\mathbb{T}^{d}\right)}$-norm and the $\|\cdot\|_{\mathcal{H}^{\beta}\left(\mathbb{T}^{d}\right)}$-norm given in (1.2) are also equivalent for $m=\beta$ because of the observation that

$$
\max \left(1,\left|k_{j}\right|\right)^{2} \leq 1+\left|k_{j}\right|^{2} \leq 2 \max \left(1,\left|k_{j}\right|\right)^{2}
$$

for all $k_{j} \in \mathbb{Z}$. In total, for $m \in \mathbb{N}$ we have the norm equivalences

$$
\|\cdot\|_{\mathcal{H}^{m}\left(\mathbb{T}^{d}\right)} \sim\|\cdot\|_{H^{m,+}\left(\mathbb{T}^{d}\right)} \sim\|\cdot\|_{H_{\text {mix }}^{m}\left(\mathbb{T}^{d}\right)},
$$

but we distinguish the related function spaces anyway, because $\mathcal{H}^{\beta}\left(\mathbb{T}^{d}\right)$ appears in results concerned with approximation error bounds, whereas $H_{\text {mix }}^{m}\left(\mathbb{T}^{d}\right)$ is considered later on when we discuss smoothness-preserving transformation mappings.

Considering furthermore the function spaces $\mathcal{A}^{\beta}\left(\mathbb{T}^{d}\right)$ as defined in (1.1), it was shown in [13, Lemma 2.2] that for $\beta \geq 0, \lambda>\frac{1}{2}$, and fixed $d \in \mathbb{N}$, there are continuous embeddings

$$
\mathcal{H}^{\beta+\lambda}\left(\mathbb{T}^{d}\right) \hookrightarrow \mathcal{A}^{\beta}\left(\mathbb{T}^{d}\right) \hookrightarrow \mathcal{A}\left(\mathbb{T}^{d}\right),
$$

and for $f \in \mathcal{A}^{\beta}\left(\mathbb{T}^{d}\right)$, we have

$$
\|f\|_{\mathcal{A}^{\beta}\left(\mathbb{T}^{d}\right)} \leq C_{d, \lambda}\|f\|_{\mathcal{H}^{\beta+\lambda}\left(\mathbb{T}^{d}\right)}
$$

with a constant $C_{d, \lambda}:=C(d, \lambda)>1$. Additionally, for each function in $\mathcal{A}\left(\mathbb{T}^{d}\right)$, there exists a continuous representative, as proven in [8, Lemma 2.1]. Later on, when we sample functions $f \in \mathcal{H}^{\beta+\lambda}\left(\mathbb{T}^{d}\right)$, we identify them with their continuous representatives given by their Fourier series $\sum_{\mathbf{k} \in \mathbb{Z}^{d}} \hat{f}_{\mathbf{k}} \mathrm{e}^{2 \pi \mathrm{ik} \cdot \circ}$, and this identification will be denoted by $f \in \mathcal{H}^{\beta+\lambda}\left(\mathbb{T}^{d}\right) \cap \mathcal{C}\left(\mathbb{T}^{d}\right)$.

2.2. Rank-1 lattices and reconstructing rank-1 lattices. Before discussing the approximation of functions $f \in \mathcal{H}^{\beta}\left(\mathbb{T}^{d}\right) \cap \mathcal{C}\left(\mathbb{T}^{d}\right)$, we recollect some related objects and observations from $[4,8,20,25]$. For each frequency set $I \subset \mathbb{Z}^{d}$, there is the difference set

$$
\mathcal{D}(I):=\left\{\mathbf{k} \in \mathbb{Z}^{d}: \mathbf{k}=\mathbf{k}_{1}-\mathbf{k}_{2} \text { with } \mathbf{k}_{1}, \mathbf{k}_{2} \in I\right\} .
$$

Furthermore, the set

$$
\Lambda(\mathbf{z}, M):=\left\{\mathbf{x}_{j}:=\left(\frac{j}{M} \mathbf{z} \bmod \mathbf{1}\right) \in \mathbb{T}^{d}: j=0,1, \ldots M-1\right\}
$$

is called a rank-1 lattice with the generating vector $\mathbf{z} \in \mathbb{Z}^{d}$ and the lattice size $M \in \mathbb{N}$, where $\mathbf{1}:=(1, \ldots, 1)^{\top} \in \mathbb{Z}^{d}$. To ensure that $\Lambda(\mathbf{z}, M)$ has exactly $M$ distinct elements, it is pointed out in [20, p. 428] that we need to assume that $M$ is coprime with at least one component of 


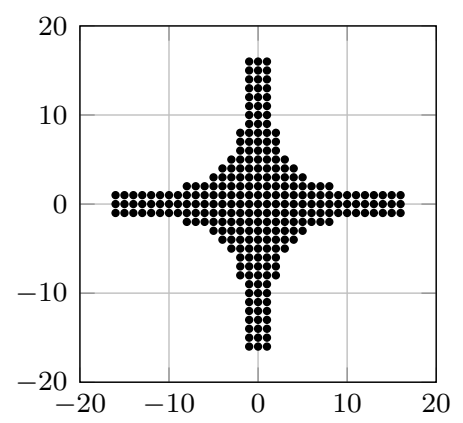

FIG. 2.1. The hyperbolic cross $I_{N}^{d}$ for $N=16$ and $d=2$.

the generating vector $\mathbf{z}$. A reconstructing rank-1 lattice $\Lambda(\mathbf{z}, M, I)$ is a rank-1 lattice $\Lambda(\mathbf{z}, M)$ for which the condition

$$
\mathbf{t} \cdot \mathbf{z} \not \equiv 0(\bmod M) \quad \text { for all } \mathbf{t} \in \mathcal{D}(I) \backslash\{\mathbf{0}\}
$$

holds. Given a reconstructing rank-1 lattice $\Lambda(\mathbf{z}, M, I)$, we have exact integration for all multivariate trigonometric polynomials $g \in \Pi_{\mathcal{D}(I)}$ (see [25]) so that

$$
\int_{\mathbb{T}^{d}} g(\mathbf{x}) \mathrm{d} \mathbf{x}=\frac{1}{M} \sum_{j=0}^{M-1} g\left(\mathbf{x}_{j}\right), \quad \mathbf{x}_{j} \in \Lambda(\mathbf{z}, M, I) .
$$

In particular, for $f \in \Pi_{I}$ and $\mathbf{k} \in I$, we have $f(\circ) \mathrm{e}^{-2 \pi \mathbf{i k} \cdot \circ} \in \Pi_{\mathcal{D}(I)}$ and

$$
\hat{f}_{\mathbf{k}}=\int_{\mathbb{T}^{d}} f(\mathbf{x}) \mathrm{e}^{-2 \pi \mathrm{i} \mathbf{k} \cdot \mathbf{x}} \mathrm{d} \mathbf{x}=\frac{1}{M} \sum_{j=0}^{M-1} f\left(\mathbf{x}_{j}\right) \mathrm{e}^{-2 \pi \mathrm{i} \mathbf{k} \cdot \mathbf{x}_{j}}, \quad \mathbf{x}_{j} \in \Lambda(\mathbf{z}, M, I) .
$$

For an arbitrary function $f \in \mathcal{H}^{\beta}\left(\mathbb{T}^{d}\right) \cap \mathcal{C}\left(\mathbb{T}^{d}\right)$ and lattice points $\mathbf{x}_{j} \in \Lambda(\mathbf{z}, M, I)$, we lose the former mentioned exactness and get approximated Fourier coefficients $\hat{f}_{\mathbf{k}}^{\Lambda} \approx \hat{f}_{\mathrm{k}}$ of the form (1.4) leading to the approximated Fourier partial sum $S_{I}^{\Lambda} f(\mathbf{x}) \approx S_{I} f(\mathbf{x})$ as given in (1.3).

2.3. Lattice-based approximation on the torus. We discuss upper bounds for certain approximation errors $\left\|f-S_{I_{N}^{d}}^{\Lambda} f\right\|$ of functions $f$ in $\mathcal{A}^{\beta}\left(\mathbb{T}^{d}\right) \cap \mathcal{C}\left(\mathbb{T}^{d}\right)$ and $\mathcal{H}^{\beta}\left(\mathbb{T}^{d}\right) \cap \mathcal{C}\left(\mathbb{T}^{d}\right)$. For this matter, the frequency sets are hyperbolic crosses $I_{N}^{d}$ as defined in (1.5) and are illustrated for $N=16$ in two dimensions in Figure 2.1. For approximation purposes the existence of reconstructing rank-1 lattices is secured by the arguments provided in [10, Corollary 1] and [13, Lemma 2.1]:

LEMMA 2.1. Let $I \subset \mathbb{Z}^{d}$ be a frequency set of finite cardinality $4 \leq|I|<\infty$ with $I \subset \mathbb{Z}^{d} \cap(-M / 2, M / 2)^{d}$. For all multivariate trigonometric polynomials $f \in \Pi_{I}$, there exists a reconstructing rank-1 lattice $\Lambda(\mathbf{z}, M, I)$ with the lattice size $M \in \mathbb{N}$ bounded by $|I| \leq M \leq|\mathcal{D}(I)| \leq|I|^{2}$, such that $\hat{f}_{\mathbf{k}}=\hat{f}_{\mathbf{k}}^{\Lambda}$. The generating vector $\mathbf{z}$ can be constructed using a component-by-component approach.

Then it is possible to prove an upper error bound for the $L_{\infty}$-approximation of functions in the subspace $\mathcal{A}^{\beta}\left(\mathbb{T}^{d}\right)$ of the Wiener Algebra, as observed in [13, Theorem 3.3]:

THEOREM 2.2. Let be given $f \in \mathcal{A}^{\beta}\left(\mathbb{T}^{d}\right) \cap \mathcal{C}\left(\mathbb{T}^{d}\right)$ with $\beta \geq 0$ and $d \in \mathbb{N}$, a hyperbolic cross $I_{N}^{d}$ with $\left|I_{N}^{d}\right|<\infty$ and $N \in \mathbb{N}$, and a reconstructing rank-1 lattice $\Lambda\left(\mathbf{z}, M, I_{N}^{d}\right)$. The 


\section{ETNA}

Kent State University and

Johann Radon Institute (RICAM)

approximation of $f$ by the approximated Fourier partial sum $S_{I_{N}^{d}}^{\Lambda} f$ leads to an approximation error that is bounded by

$$
\left\|f-S_{I_{N}^{d}}^{\Lambda} f\right\|_{L_{\infty}\left(\mathbb{T}^{d}\right)} \leq 2 N^{-\beta}\|f\|_{\mathcal{A}^{\beta}\left(\mathbb{T}^{d}\right)} .
$$

The approximation of functions in the Hilbert spaces $\mathcal{H}^{\beta}\left(\mathbb{T}^{d}\right)$ was investigated by V. N. Temlyakov; see $[13,26]$. He showed that for $\beta>1$, there exists a reconstructing rank-1 lattice generated by a vector of Korobov form $\mathbf{z}:=\left(1, z, z^{2}, \ldots, z^{d-1}\right)^{\top} \in \mathbb{Z}^{d}$ such that the $L_{2}$-truncation error is bounded above by

$$
\left\|f-S_{I_{N}^{d}}^{\Lambda} f\right\|_{L_{2}\left(\mathbb{T}^{d}\right)} \leq N^{-\beta}(\log N)^{(d-1) / 2}\|f\|_{\mathcal{H}^{\beta}\left(\mathbb{T}^{d}\right)} .
$$

A generalization of this estimate as well as an upper bound for the corresponding aliasing error is stated in [2, Theorem 2] using dyadic hyperbolic cross frequency sets and a componentby-component approach to construct the generating vector $\mathbf{z} \in \mathbb{Z}^{d}$, which generally is not of Korobov form anymore. However, every dyadic hyperbolic cross is embedded in a non-dyadic one; see [30, Lemma 2.29]. Thus, the error estimates are easily translated in terms of nondyadic hyperbolic crosses $I_{N}^{d}$ (see [30, Theorem 2.30]), and we are particularly interested in the following special case:

THEOREM 2.3. Let be given $\beta>\frac{1}{2}$, the dimension $d \in \mathbb{N}$, a function $f \in \mathcal{H}^{\beta}\left(\mathbb{T}^{d}\right) \cap$ $\mathcal{C}\left(\mathbb{T}^{d}\right)$, a hyperbolic cross $I_{N}^{d}$ with $N \geq 2^{d+1}$, and a reconstructing rank-1 lattice $\Lambda\left(\mathbf{z}, M, I_{N}^{d}\right)$. Then we have

$$
\left\|f-S_{I_{N}^{d}}^{\Lambda} f\right\|_{L_{2}\left(\mathbb{T}^{d}\right)} \leq C_{d, \beta} N^{-\beta}(\log N)^{(d-1) / 2}\|f\|_{\mathcal{H}^{\beta}\left(\mathbb{T}^{d}\right)}
$$

with some constant $C_{d, \beta}:=C(d, \beta)>0$.

As highlighted earlier in (2.2), for $\beta=m \in \mathbb{N}$, the norms $\|\cdot\|_{\mathcal{H}^{\beta}\left(\mathbb{T}^{d}\right)}$ and $\|\cdot\|_{H_{\text {mix }}^{m}\left(\mathbb{T}^{d}\right)}$ are equivalent. Eventually, we utilize this norm equivalence in order to apply the above approximation error bounds for functions $f$ in the Sobolev space $H_{\text {mix }}^{m}\left(\mathbb{T}^{d}\right)$ that are characterized by their derivatives.

3. Torus-to- $\mathbb{R}$ transformation mappings. Change of variables were discussed, for example, in $[1,23]$ and were used for high-dimensional integration in, e.g., $[16,18]$. In this chapter we define transformations $\psi:\left(-\frac{1}{2}, \frac{1}{2}\right)^{d} \rightarrow \mathbb{R}^{d}$ and provide examples that will reappear later in this paper. Afterwards, we describe the weighted Hilbert spaces $L_{2}\left(\mathbb{R}^{d}, \omega\right)$ with weight functions $\omega: \mathbb{R}^{d} \rightarrow[0, \infty)$ and investigate their structure. Then we prove sufficient conditions for $\psi$ and $\omega$ such that an initially chosen $h \in L_{2}\left(\mathbb{R}^{d}, \omega\right) \cap H_{\text {mix }}^{m}\left(\mathbb{R}^{d}\right)$ is transformed by the change of variables $\psi$ into a function that is lying in a Sobolev space $H_{\text {mix }}^{m}\left(\mathbb{T}^{d}\right)$ of mixed natural smoothness order $m \in \mathbb{N}_{0}$. Eventually, we show that with an incorporated transformation $\psi$, we still have upper bounds for certain approximation errors on $\mathbb{R}^{d}$, which are based on the already established error bounds with respect to the $L_{\infty}\left(\mathbb{T}^{d}\right)$ - and $L_{2}\left(\mathbb{T}^{d}\right)$-norms recalled in Theorems 2.2 and 2.3, respectively.

3.1. Transformations to $\mathbb{R}^{d}$. We call a map $\psi:\left(-\frac{1}{2}, \frac{1}{2}\right) \rightarrow \mathbb{R}$ a transformation or change of variables if it is continuously differentiable, strictly increasing, odd, and we have

$$
\lim _{x \rightarrow-\frac{1}{2}} \psi(x)=-\infty, \quad \lim _{x \rightarrow \frac{1}{2}} \psi(x)=\infty .
$$

We denote its first derivative by $\psi^{\prime}(x):=\frac{\mathrm{d}}{\mathrm{d} x}[\psi](x)$. The corresponding inverse transformation is also continuously differentiable, increasing, and it is denoted by $\psi^{-1}: \mathbb{R} \rightarrow\left(-\frac{1}{2}, \frac{1}{2}\right)$ in the 
sense of $y=\psi(x) \Leftrightarrow x=\psi^{-1}(y)$. We call the derivative of the inverse transformation the density function of $\psi$, which we denote as

$$
\varrho(y):=\left(\psi^{-1}\right)^{\prime}(y)=\frac{1}{\psi^{\prime}\left(\psi^{-1}(y)\right)},
$$

and for which we have $\varrho(y) \geq 0$ for all $y \in \mathbb{R}$. Furthermore, we have $\lim _{y \rightarrow-\infty} \psi^{-1}(y)=-\frac{1}{2}$ and $\lim _{y \rightarrow \infty} \psi^{-1}(y)=\frac{1}{2}$. We note that $\varrho$ is a bounded function with

$$
\|\varrho\|_{L_{1}(\mathbb{R})}=\int_{-\infty}^{\infty} \varrho(y) \mathrm{d} y=1
$$

For multivariate transformations we let

$$
\psi(\mathbf{x}):=\left(\psi_{1}\left(x_{1}\right), \ldots, \psi_{d}\left(x_{d}\right)\right)^{\top} \quad \text { and } \quad \psi^{\prime}(\mathbf{x}):=\prod_{j=1}^{d} \psi_{j}^{\prime}\left(x_{j}\right)
$$

with $\mathbf{x}=\left(x_{1}, \ldots, x_{d}\right)^{\top} \in\left(-\frac{1}{2}, \frac{1}{2}\right)^{d}$, where we may use different transformations $\psi_{j}$ in each direction. Similarly, we let $\psi^{-1}(\mathbf{y}):=\left(\psi_{1}^{-1}\left(y_{1}\right), \ldots, \psi_{d}^{-1}\left(y_{d}\right)\right)^{\top}$ and

$$
\varrho(\mathbf{y}):=\prod_{j=1}^{d} \varrho_{j}\left(y_{j}\right)
$$

with $\mathbf{y}=\left(y_{1}, \ldots, y_{d}\right)^{\top} \in \mathbb{R}^{d}$.

Later on, we consider families of parameterized transformations

$$
\psi(\mathbf{x}, \boldsymbol{\eta}):=\left(\psi_{1}\left(x_{1}, \eta_{1}\right), \ldots, \psi_{d}\left(x_{d}, \eta_{d}\right)\right)^{\top}
$$

with $\boldsymbol{\eta}=\left(\eta_{1}, \ldots, \eta_{d}\right)^{\top} \in \mathbb{R}^{d}$. We only consider parametrizations for which the transformation $\psi$, its inverse $\psi^{-1}$, and the density function $\varrho$ fit into the given definitions above despite being affected by the parameter $\boldsymbol{\eta}$. On several occasions throughout this paper we will replace the transformations $\psi(x)$ by

$$
\psi(\mathbf{x}, \boldsymbol{\eta}):=\boldsymbol{\eta} \cdot \psi(\mathbf{x})
$$

with $\boldsymbol{\eta} \in(0, \infty)^{d}$. As the transformations are going to be composed with functions defined on $\mathbb{R}^{d}$, the parameter $\boldsymbol{\eta}$ may influence the smoothness of the resulting transformed functions, which we will discuss in depth later on. For now we omit the parameter in the notation for simplicity and proceed to just write $\psi(\circ)$ until we actually consider particular parameterized families of the form (3.4) or (3.5).

3.2. Exemplary transformations. We list some feasible univariate transformations $\psi$ with either an algebraic or an exponential density function $\varrho$, some of which were suggested in the literature; see, e.g., [1, Section 17.6] and [23, Section 7.5]. With the remark about (3.4) in mind, we list these transformations here in their univariate non-parameterized form with $\eta=1$ and $\psi(x)=\psi(x, 1)$, for simplicity. Later on, when we fix a particular family of parameterized transformations $\psi(\circ, \eta), \eta \in \mathbb{R}$, we recall these definitions accordingly. tions:

Let $x \in\left(-\frac{1}{2}, \frac{1}{2}\right)$ and $y \in \mathbb{R}$. We are particularly interested in the following transforma- 
- algebraic transformation:

$$
\begin{aligned}
\psi(x) & =\frac{2 x}{\left(1-4 x^{2}\right)^{\frac{1}{2}}}, & \psi^{\prime}(x) & =\frac{2}{\left(1-4 x^{2}\right)^{\frac{3}{2}}}, \\
\psi^{-1}(y) & =\frac{y}{2\left(1+y^{2}\right)^{\frac{1}{2}}}, & \varrho(y) & =\frac{1}{2\left(1+y^{2}\right)^{\frac{3}{2}}},
\end{aligned}
$$

- tangent transformation:

$$
\begin{aligned}
\psi(x) & =\tan (\pi x), & \psi^{\prime}(x) & =\frac{\pi}{\cos ^{2}(\pi x)}, \\
\psi^{-1}(y) & =\frac{1}{\pi} \arctan (y), & \varrho(y) & =\frac{1}{\pi}\left(\frac{1}{1+y^{2}}\right),
\end{aligned}
$$

- error function transformation:

$$
\begin{aligned}
\psi(x) & =\operatorname{erf}^{-1}(2 x), & \psi^{\prime}(x) & =\sqrt{\pi} \mathrm{e}^{\left(\operatorname{erf}^{-1}(2 x)\right)^{2},}, \\
\psi^{-1}(y) & =\frac{1}{2} \operatorname{erf}(y), & \varrho(y) & =\frac{1}{\sqrt{\pi}} \mathrm{e}^{-y^{2}},
\end{aligned}
$$

with the error function

$$
\operatorname{erf}(x)=\frac{1}{\sqrt{\pi}} \int_{-x}^{x} \mathrm{e}^{-t^{2}} \mathrm{~d} t, \quad x \in \mathbb{R},
$$

and $\operatorname{erf}^{-1}(\circ)$ denoting the inverse error function,

- logarithmic transformation:

$$
\begin{aligned}
\psi(x) & =\frac{1}{2} \log \left(\frac{1+2 x}{1-2 x}\right)=\tanh ^{-1}(2 x), & \psi^{\prime}(x) & =\frac{2}{1-4 x^{2}} \\
\psi^{-1}(y) & =\frac{1}{2}\left(\frac{\mathrm{e}^{2 y}-1}{\mathrm{e}^{2 y}+1}\right)=\frac{1}{2} \tanh (y), & \varrho(y) & =\frac{2 \mathrm{e}^{2 y}}{\left(\mathrm{e}^{2 y}+1\right)^{2}} .
\end{aligned}
$$

For a side-by-side comparison of their individual slope, see Figure 3.1.

3.3. Weighted Hilbert spaces on $\mathbb{R}$. We describe the structure of the weighted $L_{2}(\mathbb{R}, \omega)$ function spaces as defined in (2.1). In this section the weight function $\omega: \mathbb{R} \rightarrow[0, \infty)$ remains unspecified. However, similarly to the generalization (3.4) of transformations $\psi$ defined in (3.1), we will later on consider families of non-negative parameterized weight functions $\omega(\circ, \mu)$ with $\mu \in \mathbb{R}$ for the purpose of controlling the smoothness of functions in $L_{2}(\mathbb{R}, \omega(\circ, \mu)) \cap H^{m}(\mathbb{R})$ and of the corresponding transformed functions on the torus $\mathbb{T}$. Analogously, families of multivariate parameterized weight functions are defined as

$$
\omega(\mathbf{y}, \boldsymbol{\mu}):=\prod_{j=1}^{d} \omega_{j}\left(y_{j}, \mu_{j}\right), \quad \mathbf{y}, \boldsymbol{\mu} \in \mathbb{R}^{d},
$$

with univariate weight functions $\omega_{j}\left(\circ, \mu_{j}\right): \mathbb{R} \rightarrow[0, \infty)$.

For now we remain in the univariate setting. The system $\left\{\varphi_{k}\right\}_{k \in \mathbb{Z}}$ of weighted exponential functions

$$
\varphi_{k}(y):=\sqrt{\frac{\varrho(y)}{\omega(y)}} \mathrm{e}^{2 \pi \mathrm{i} k \psi^{-1}(y)}, \quad y \in \mathbb{R}
$$




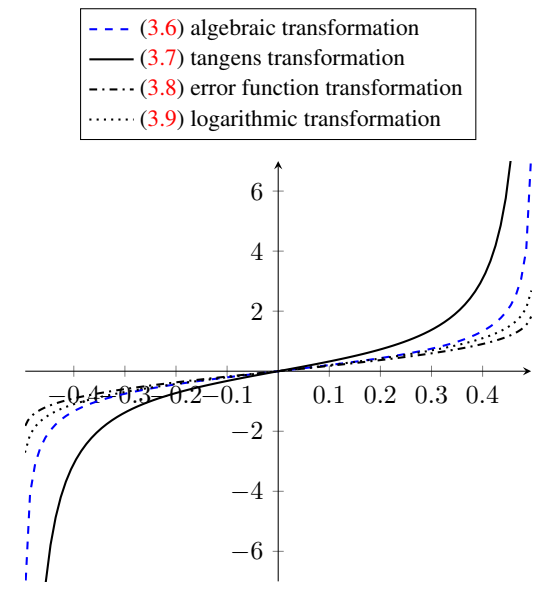

FIG. 3.1. Plots of exemplary transformations (3.6)-(3.9).

forms an orthogonal system with respect to the scalar product

$$
\left(h_{1}, h_{2}\right)_{L_{2}(\mathbb{R}, \omega)}:=\int_{\mathbb{R}} \omega(y) h_{1}(y) \overline{h_{2}(y)} \mathrm{d} y,
$$

and for $k_{1}, k_{2} \in \mathbb{Z}$ we have

$$
\left(\varphi_{k_{1}}, \varphi_{k_{2}}\right)_{L_{2}(\mathbb{R}, \omega)}=\delta_{k_{1}, k_{2}} .
$$

The weighted scalar product (3.12) induces the norm

$$
\|h\|_{L_{2}(\mathbb{R}, \omega)}:=\sqrt{(h, h)_{L_{2}(\mathbb{R}, \omega)}},
$$

and in a natural way we have Fourier coefficients of the form

$$
\hat{h}_{k}:=\left(h, \varphi_{k}\right)_{L_{2}(\mathbb{R}, \omega)}=\int_{\mathbb{R}} h(y) \sqrt{\varrho(y) \omega(y)} \mathrm{e}^{-2 \pi \mathrm{i} k \psi^{-1}(y)} \mathrm{d} y
$$

as well as the corresponding Fourier partial sum for $I \subset \mathbb{Z}$ given by

$$
S_{I} h(y):=\sum_{k \in I} \hat{h}_{k} \varphi_{k}(y)
$$

EXAMPLE 3.1.

- For the algebraic transformation (3.6) with the density $\varrho(y)=\frac{1}{2\left(1+y^{2}\right)^{\frac{3}{2}}}$ and the parameterized weight function

$$
\omega(y, \mu)=\left(\frac{1}{1+y^{2}}\right)^{\mu}, \quad \mu \in \mathbb{R}
$$

the orthogonal system functions $\varphi_{k}$ as in (3.11) are of the form

$$
\varphi_{k}(y)=\sqrt{\frac{1}{2}\left(\frac{1}{1+y^{2}}\right)^{\frac{3}{2}-\mu}} \mathrm{e}^{\pi \mathrm{i} k \frac{y}{\sqrt{1+y^{2}}}} .
$$

The graphs of their real and imaginary parts of these $\varphi_{k}$ are shown in Figure 3.2 for $\mu=2$ and $k=0,1,2,3$. 

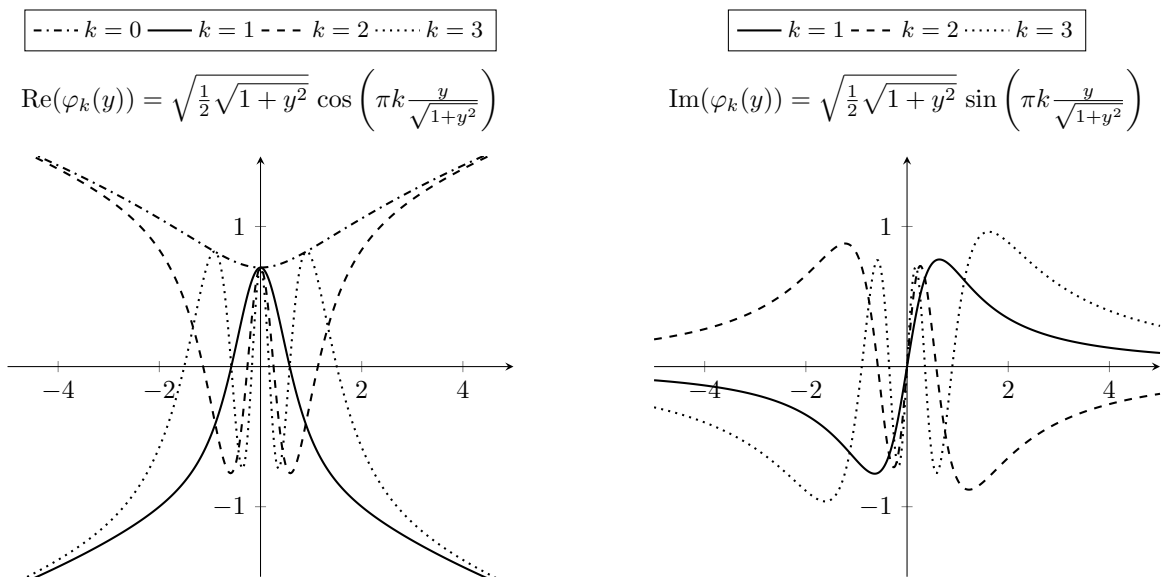

FIG. 3.2. Real and imaginary part of the weighted exponential functions $\varphi_{k}, k=0,1,2,3$, in (3.11) with the density function $\varrho$ of the algebraic transformation (3.6) and the algebraic parameterized weight function $\omega(y, \mu)$ as given in (5.2) for fixed $\mu=2$.

- For the error function transformation (3.8) with the density $\varrho(y)=\frac{1}{\sqrt{\pi}} \mathrm{e}^{-y^{2}}$ and the Gaussian weight function

$$
\omega(y, \mu)=\frac{1}{\sqrt{\pi}} \mathrm{e}^{-\mu^{2} y^{2}}, \quad \mu \in \mathbb{R},
$$

the orthogonal system functions $\varphi_{k}$ as in (3.11) are of the form

$$
\varphi_{k}(y)=\mathrm{e}^{\frac{1}{2}\left(\mu^{2}-1\right) y^{2}+\pi \mathrm{i} k \operatorname{erf}(y)},
$$

with graphs of their real and imaginary parts for $\mu=\sqrt{2}$ and $k=0,1,2,3$ displayed in Figure 3.3. The corresponding weighted scalar product (3.12) reads as

$$
\left(h_{1}, h_{2}\right)_{L_{2}(\mathbb{R}, \omega(\circ, \mu))}=\frac{1}{\sqrt{\pi}} \int_{\mathbb{R}} \mathrm{e}^{-\mu^{2} y^{2}} h_{1}(y) \overline{h_{2}(y)} \mathrm{d} y .
$$

3.4. Smoothness properties of composed functions in Sobolev spaces. In this section we discuss the smoothness of univariate functions $h$ defined on $\mathbb{R}$ and of their resulting transformed versions $f$ on the torus $\mathbb{T}$. In [16] the authors use a change of variables for integration problems with respect to a family of integrands with bounded $L_{p}$-norm of mixed first-order partial derivatives with $1 \leq p \leq \infty$ and provide sufficient conditions such that the transformed integrand belongs to a Sobolev space of mixed smoothness order one. We will propose a specific set of sufficient conditions for $\psi$ and $\omega$ such that $f \in H_{\text {mix }}^{m}\left(\mathbb{T}^{d}\right)$ with $m \in \mathbb{N}_{0}$. These conditions are stated for both univariate and multivariate functions. Afterwards, we utilize the norm equivalence of the Sobolev space $H_{\text {mix }}^{m}\left(\mathbb{T}^{d}\right)$ and the subspace $\mathcal{H}^{\beta}\left(\mathbb{T}^{d}\right)$ of the Wiener Algebra $\mathcal{A}\left(\mathbb{T}^{d}\right)$ for $m=\beta$ as described in (2.2) and combine it with the embedding $\mathcal{H}^{\beta+\lambda}\left(\mathbb{T}^{d}\right) \hookrightarrow \mathcal{A}^{\beta}\left(\mathbb{T}^{d}\right)$ in (2.3) for all $\lambda>\frac{1}{2}$ in order to discuss high-dimensional approximation problems in which we apply rank-1 lattice-based fast Fourier approximation methods. Throughout this section we still omit the parameters $\boldsymbol{\eta}, \boldsymbol{\mu} \in \mathbb{R}^{d}$ in the notation of the transformations $\psi$ and the weight functions $\omega$, as outlined in (3.4) and (3.10).

For now we consider univariate transformed functions $f \in L_{2}(\mathbb{T})$ of the form

$$
f(x):=h(\psi(x)) \sqrt{\omega(\psi(x)) \psi^{\prime}(x)}, \quad x \in \mathbb{T},
$$



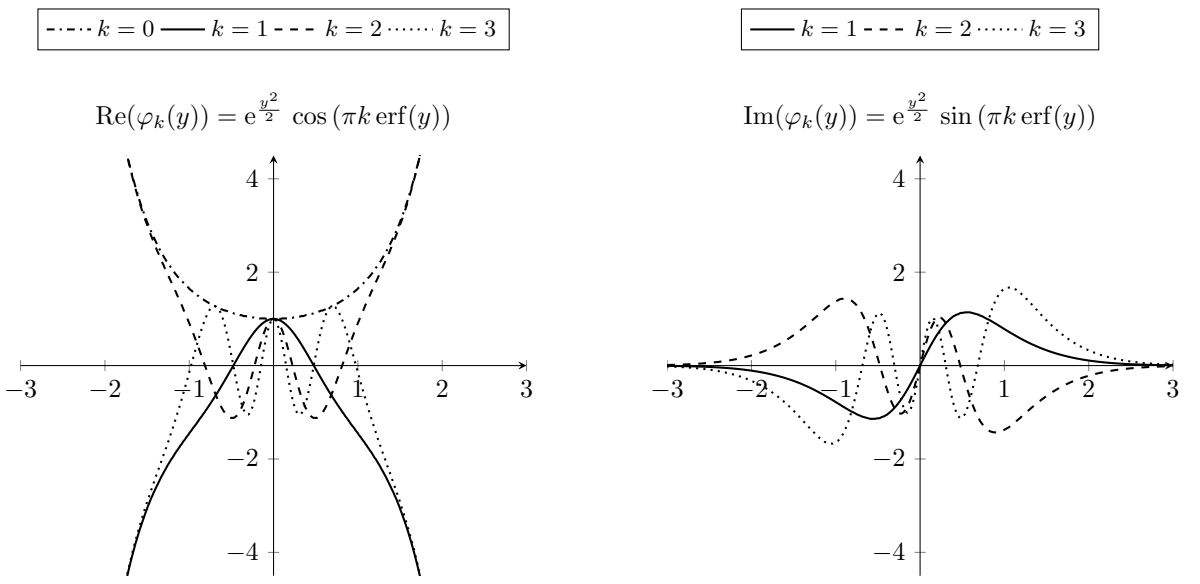

FIG. 3.3. Real and imaginary part of the weighted exponential functions $\varphi_{k}, k=0,1,2,3$, in (3.11) with the density function $\varrho$ of the error function transformation (3.8) and the parameterized Gaussian weight function $\omega(y, \mu)$ as given in (5.8) for fixed $\mu=\sqrt{2}$.

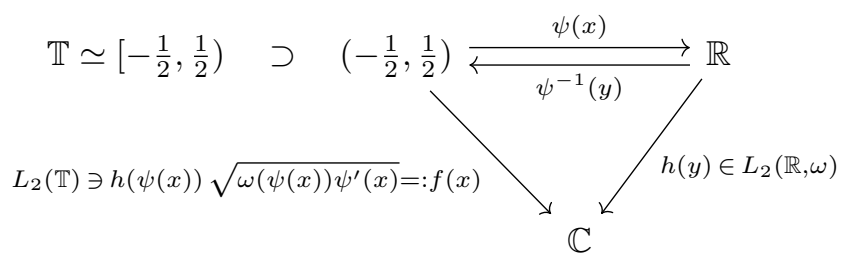

FIG. 3.4. Scheme of the relation between $f$ and $h$ caused by a transformation $\psi$.

which are the result of applying the change of variables $y=\psi(x)$ as defined in (3.1) to a function $h \in L_{2}(\mathbb{R}, \omega)$ and for which we have the identity

$$
\|h\|_{L_{2}(\mathbb{R}, \omega)}^{2}=\int_{\mathbb{R}}|h(y)|^{2} \omega(y) \mathrm{d} y=\int_{\mathbb{T}}|h(\psi(x))|^{2} \omega(\psi(x)) \psi^{\prime}(x) \mathrm{d} x=\|f\|_{L_{2}(\mathbb{T})}^{2},
$$

schematically illustrated in Figure 3.4.

REMARK 3.2. The transformed functions $f$ as given in (3.17) are generally not in $L_{2}(\mathbb{T})$ for all transformations $\psi$. We will consider families of transformations $\psi(\circ, \eta), \eta \in \mathbb{R}$, as in (3.4) and families of weight functions $\omega(0, \mu), \mu \in \mathbb{R}$, as in (3.10). Generally, there exist restrictions for the range of feasible parameters $\eta, \mu \in \mathbb{R}$ for which the transformed functions $f(\circ, \eta, \mu)$ as in (3.17) are in $L_{2}(\mathbb{T})$. Later on, we present examples with multivariate functions $h \in L_{2}\left(\mathbb{R}^{d}, \omega(\circ, \boldsymbol{\mu})\right) \cap H_{\text {mix }}^{m}\left(\mathbb{R}^{d}\right)$ and a fixed family of transformations $\psi(\circ, \boldsymbol{\eta}), \boldsymbol{\eta} \in \mathbb{R}$, and calculate parameter ranges of $\boldsymbol{\eta}$ and $\boldsymbol{\mu}$ for which the transformed functions $f$ are in $H_{\text {mix }}^{m}\left(\mathbb{T}^{d}\right)$ for $m \in \mathbb{N}_{0}$.

It is generally rather difficult to verify if such transformed functions $f$ are in $H^{m}(\mathbb{T})$ for some fixed $m \in \mathbb{N}_{0}$ by calculating the individual $L_{2}(\mathbb{T})$-norms within the Sobolev norm $\|f\|_{H^{m}(\mathbb{T})}$. Therefore we propose two different sets of sufficient conditions such that $f \in H^{m}(\mathbb{T})$, with $m \in \mathbb{N}_{0}$, by utilizing the product structure of the functions $f$ in (3.17). At first we state conditions for $h \in L_{2}(\mathbb{R}, \omega)$, the weight function $\omega$, and the transformation $\psi$ 
to preserve a certain degree of smoothness $m$ of $h$ under the transformation with $\psi$, which slightly simplifies the problem of the difficult evaluation of $L_{2}(\mathbb{T})$-integrals.

THEOREM 3.3. Let be given $m \in \mathbb{N}_{0}$, a transformation $\psi:\left(-\frac{1}{2}, \frac{1}{2}\right) \rightarrow \mathbb{R}$ as defined in (3.1), a function $h \in L_{2}(\mathbb{R}, \omega)$ with a weight function $\omega: \mathbb{R} \rightarrow[0, \infty)$, and the corresponding transformed functions $f$ of the form (3.17). We have that $f \in H^{m}(\mathbb{T})$ if either for all $k=0,1, \ldots, m$

$$
\frac{\mathrm{d}^{k}}{\mathrm{~d} x^{k}}[h \circ \psi](x) \in L_{\infty}(\mathbb{T}) \quad \text { and } \quad \frac{\mathrm{d}^{k}}{\mathrm{~d} x^{k}}\left[\sqrt{(\omega \circ \psi) \psi^{\prime}}\right](x) \in L_{2}(\mathbb{T}),
$$

or if, for all $k=0,1, \ldots, m$,

$$
\frac{\mathrm{d}^{k}}{\mathrm{~d} x^{k}}[h \circ \psi](x) \in L_{2}(\mathbb{T}) \quad \text { and } \quad \frac{\mathrm{d}^{k}}{\mathrm{~d} x^{k}}\left[\sqrt{(\omega \circ \psi) \psi^{\prime}}\right](x) \in L_{\infty}(\mathbb{T}) .
$$

Proof. Let $f$ be of the form (3.17) and $k=0,1, \ldots, m$. Using the well-known generalized Leibniz rule for the $k$-th derivative of a product of two functions leads to

$$
\left\|\frac{\mathrm{d}^{k}}{\mathrm{~d} x^{k}}[f](x)\right\|_{L_{2}(\mathbb{T})} \leq \sum_{\ell=0}^{k}\left(\begin{array}{l}
k \\
\ell
\end{array}\right)\left\|\frac{\mathrm{d}^{\ell}}{\mathrm{d} x^{\ell}}[h \circ \psi](x) \frac{\mathrm{d}^{k-\ell}}{\mathrm{d} x^{k-\ell}}\left[\sqrt{(\omega \circ \psi) \psi^{\prime}}\right](x)\right\|_{L_{2}(\mathbb{T})} .
$$

Now we either estimate

$$
\left\|\frac{\mathrm{d}^{k}}{\mathrm{~d} x^{k}}[f](x)\right\|_{L_{2}(\mathbb{T})} \leq \sum_{\ell=0}^{k}\left(\begin{array}{c}
k \\
\ell
\end{array}\right)\left\|\frac{\mathrm{d}^{\ell}}{\mathrm{d} x^{\ell}}[h \circ \psi](x)\right\|_{L_{\infty}(\mathbb{T})}\left\|\frac{\mathrm{d}^{k-\ell}}{\mathrm{d} x^{k-\ell}}\left[\sqrt{(\omega \circ \psi) \psi^{\prime}}\right](x)\right\|_{L_{2}(\mathbb{T})}
$$

or

$$
\left\|\frac{\mathrm{d}^{k}}{\mathrm{~d} x^{k}}[f](x)\right\|_{L_{2}(\mathbb{T})} \leq \sum_{\ell=0}^{k}\left(\begin{array}{l}
k \\
\ell
\end{array}\right)\left\|\frac{\mathrm{d}^{\ell}}{\mathrm{d} x^{\ell}}[h \circ \psi](x)\right\|_{L_{2}(\mathbb{T})}\left\|\frac{\mathrm{d}^{k-\ell}}{\mathrm{d} x^{k-\ell}}\left[\sqrt{(\omega \circ \psi) \psi^{\prime}}\right](x)\right\|_{L_{\infty}(\mathbb{T})} .
$$

If the $\|\cdot\|_{L_{2}(\mathbb{T})}$-norms of $k$-th derivatives of $f$ are finite for all $k=0,1, \ldots, m$, then their sum, i.e., the $H^{m}(\mathbb{T})$-norm, is finite, too.

Now, we derive a set of sufficient $L_{\infty}$-conditions for $\psi$ and $\omega$ that ensure that a function $h \in L_{2}(\mathbb{R}, \omega) \cap H^{m}(\mathbb{R})$ can be transformed by $\psi$ into an $f \in H^{m}(\mathbb{T})$ of the form (3.17). This eliminates the necessity to evaluate $L_{2}$-integrals of the various derivatives of $f$. Furthermore, once we consider particular parameterized families of transformations $\psi(\circ, \eta)$ and families of weight functions $\omega(\circ, \mu)$, these conditions enable us, for each smoothness order $m \in \mathbb{N}$, to explicitly calculate how large the parameters $\eta, \mu \in \mathbb{R}$ have to be in order to preserve the fixed degree of smoothness $m$ when transforming $h \in L_{2}(\mathbb{R}, \omega(\circ, \mu)) \cap H^{m}(\mathbb{R})$ into $f \in H^{m}(\mathbb{T})$ via $\psi(\circ, \eta)$.

To simplify the notation, we alternate between equivalent expressions for derivatives of the appearing functions, and for improved readability, we write explicit arguments within certain norms. We denote the $k$-th derivative of a function $f(x)$ with respect to $x$ by either $\frac{\mathrm{d}^{k}}{\mathrm{~d} x^{k}}[f](x)$ or $f^{(k)}(x)$, and for $k=1,2,3$, we sometimes use the notation $f^{\prime}(x), f^{\prime \prime}(x)$, and $f^{\prime \prime \prime}(x)$.

THEOREM 3.4. Let be given $m \in \mathbb{N}_{0}$, a transformation $\psi:\left(-\frac{1}{2}, \frac{1}{2}\right) \rightarrow \mathbb{R}$ as defined in (3.1) with the density function $\varrho$ of $\psi$ as in (3.2), a function $h \in L_{2}(\mathbb{R}, \omega) \cap H^{m}(\mathbb{R})$ with a non-negative weight function $\omega: \mathbb{R} \rightarrow[0, \infty)$, and the corresponding transformed functions $f$ of the form (3.17). 
If for all $\ell=0,1, \ldots, m$ we have

$$
\begin{gathered}
\frac{\mathrm{d}^{\ell}}{\mathrm{d} y^{\ell}}[\varrho](y) \in \mathcal{C}_{0}(\mathbb{R}), \quad \frac{\mathrm{d}^{\ell}}{\mathrm{d} x^{\ell}}[\psi](x) \in \mathcal{C}((-1 / 2,1 / 2)), \\
\text { and } \max _{k=0, \ldots, \ell}\left\|\frac{\mathrm{d}^{\ell-k}}{\mathrm{~d} x^{\ell-k}}\left[\sqrt{(\omega \circ \psi) \psi^{\prime}}\right](x) \psi^{\prime}(x)^{\max \left(-\frac{1}{2}, 2 k-\frac{3}{2}\right)}\right\|_{L_{\infty}(\mathbb{T})}<\infty,
\end{gathered}
$$

then we have $f \in H^{m}(\mathbb{T})$.

Proof. For $h \in L_{2}(\mathbb{R}, \omega) \cap H^{m}(\mathbb{R})$ with $m \in \mathbb{N}_{0}$ and a transformation $\psi$ as defined in (3.1), we consider the function $f$ given in (3.17). In order to prove that $f \in H^{m}(\mathbb{T}$ ), we have to show that $\left\|\frac{\mathrm{d}^{n}}{\mathrm{~d} x^{n}}[f](x)\right\|_{L_{2}(\mathbb{T})}<\infty$ for all $n=0,1, \ldots, m$. We present the arguments for $n=m$, and they are applicable in the same way for $n=0,1, \ldots, m-1$, too. We consider $\left\|\frac{\mathrm{d}^{m}}{\mathrm{~d} x^{m}}[f](x)\right\|_{L_{2}(\mathbb{T})}$ and apply the generalized Leibniz rule as in (3.18), so that we now have to ensure that

$$
\left\|\frac{\mathrm{d}^{k}}{\mathrm{~d} x^{k}}[h \circ \psi](x) \frac{\mathrm{d}^{m-k}}{\mathrm{~d} x^{m-k}}\left[\sqrt{(\omega \circ \psi) \psi^{\prime}}\right](x)\right\|_{L_{2}(\mathbb{T})}<\infty,
$$

for all $k=0, \ldots, m$. We leave $h \circ \psi$ in the term corresponding to $k=0$ untouched for now. For $k=1, \ldots, m$ we use the Faá di Bruno formula to write the $k$-th derivative of the composition of the functions $h$ and $\psi$ as

$$
\frac{\mathrm{d}^{k}}{\mathrm{~d} x^{k}}[h \circ \psi](x)=\sum_{\ell=1}^{k} h^{(\ell)}(\psi(x)) \cdot B_{k, \ell}\left(\psi^{\prime}(x), \psi^{\prime \prime}(x), \ldots, \psi^{(k-\ell+1)}(x)\right),
$$

with the well-known Bell polynomials $B_{k, \ell}$, for $k, \ell \in \mathbb{N}_{0}$, given by

$$
B_{k, \ell}(\mathbf{z}):=\sum_{\substack{j_{1}+j_{2}+\ldots+j_{k-\ell+1}=\ell, j_{1}+2 j_{2}+\ldots+(k-\ell+1) j_{k-\ell+1}=k}} \frac{\ell !}{j_{1} ! \cdot \ldots \cdot j_{k-\ell+1} !} \prod_{r=1}^{k-\ell+1}\left(\frac{z_{r}}{r !}\right)^{j_{r}}
$$

with $\mathbf{z}=\left(z_{1}, \ldots, z_{k-\ell+1}\right)^{\top}$. By differentiating both sides of $\psi^{-1}(\psi(x))=x$, we obtain

$$
\psi^{\prime}(x)=\frac{1}{\varrho(\psi(x))}, \quad \psi^{\prime \prime}(x)=-\frac{\varrho^{\prime}(\psi(x)) \psi^{\prime}(x)}{\varrho(\psi(x))^{2}}=-\varrho^{\prime}(\psi(x)) \psi^{\prime}(x)^{3} .
$$

Based on this we also observe that, for $k \in \mathbb{N}$,

$$
\frac{\mathrm{d}}{\mathrm{d} x}\left[\left(\psi^{\prime}\right)^{k}\right](x)=k \psi^{\prime}(x)^{k-1} \psi^{\prime \prime}(x)=-k \psi^{\prime}(x)^{k+2} \varrho^{\prime}(\psi(x)) .
$$

Hence, the $k$-th derivative of $\psi$ can be expressed solely in terms of powers of $\psi^{\prime}$ and the first $(k-1)$ derivatives of $\varrho$ by repeated insertion of the expression of $\psi^{\prime \prime}$. Formula (3.21) implies that the highest appearing power of $\psi^{\prime}$ increases by 2 with each differentiation. For example,

$$
\begin{aligned}
& \psi^{\prime \prime \prime}(x)=\psi^{\prime}(x)^{5}\left(-\frac{\varrho^{\prime \prime}(\psi(x))}{\psi^{\prime}(x)}+3 \varrho^{\prime}(\psi(x))\right), \\
& \psi^{(4)}(x)=\psi^{\prime}(x)^{7}\left(-\frac{\varrho^{\prime \prime \prime}(\psi(x))}{\psi^{\prime}(x)^{2}}+\frac{4 \varrho^{\prime \prime}(\psi(x)) \varrho^{\prime}(\psi(x))+6 \varrho^{\prime}(\psi(x))}{\psi^{\prime}(x)}-15 \varrho^{\prime}(\psi(x))^{3}\right) .
\end{aligned}
$$

We note that each derivative of $\psi$ is bounded, based on the fact that $\varrho$ is by definition in $\mathcal{C}_{0}(\mathbb{R})$. Hence, $\varrho \circ \psi=1 / \psi^{\prime} \in \mathcal{C}(\mathbb{T})$, and any power of $1 / \psi^{\prime}$ is bounded, too. Additionally, we have 
assumed that the first $k$ derivatives of $\varrho$ are in $\mathcal{C}_{0}(\mathbb{R})$, too. Therefore, with constants $C_{k}>0$ and $C>0$, for all $k \in \mathbb{N}$, we can estimate

$$
\left|\frac{\mathrm{d}^{k}}{\mathrm{~d} x^{k}}[\psi](x)\right| \leq C_{k}\left|\psi^{\prime}(x)\right|^{2 k-1},
$$

and for the Bell polynomials $B_{k, \ell}$ in (3.20) we then estimate

$$
\begin{aligned}
& \left|B_{k, \ell}\left(\psi^{\prime}(x), \psi^{\prime \prime}(x), \ldots, \psi^{(k-\ell+1)}(x)\right)\right| \\
& \quad \leq C \cdot B_{k, \ell}\left(\left|\psi^{\prime}(x)\right|,\left|\psi^{\prime}(x)\right|^{3}, \ldots,\left|\psi^{\prime}(x)\right|^{2(k-\ell+1)-1}\right) .
\end{aligned}
$$

The Bell polynomials are defined according to the rules to partition a number $k \in \mathbb{N}$ into a sum of $\ell \in\{1,2, \ldots, k\}$ natural numbers $j_{1}, \ldots, j_{\ell} \in \mathbb{N}$ leading to the identities

$$
\begin{array}{r}
j_{1}+j_{2}+j_{3}+\ldots+j_{k-\ell+1}=\ell, \\
j_{1}+2 j_{2}+3 j_{3}+\ldots+(k-\ell+1) j_{k-\ell+1}=k .
\end{array}
$$

Subtracting the first rule from two times the second rule results in the equation

$$
j_{1}+3 j_{2}+5 j_{3}+\ldots+(2(k-\ell+1)-1) j_{k-\ell+1}=2 k-\ell,
$$

which reveals that in the polynomials $B_{k, \ell}\left(\left|\psi^{\prime}(x)\right|,\left|\psi^{\prime}(x)\right|^{3}, \ldots,\left|\psi^{\prime}(x)\right|^{2(k-\ell+1)-1}\right)$, as defined in (3.22), the highest appearing power of $\left|\psi^{\prime}\right|$ is $2 k-1$ for $\ell=1$. By extracting $\left|\psi^{\prime}(x)\right|^{2 k-1}$ from each $B_{k, \ell}$, the remaining polynomials consist only of powers of $1 / \psi^{\prime}$, which are all bounded. Hence, in (3.22) we further estimate

$$
\begin{aligned}
& \left|B_{k, \ell}\left(\psi^{\prime}(x), \psi^{\prime \prime}(x), \ldots, \psi^{(k-\ell+1)}(x)\right)\right| \\
& \quad \leq C\left|\psi^{\prime}(x)^{2 k-1} \frac{B_{k, \ell}\left(\left|\psi^{\prime}(x)\right|,\left|\psi^{\prime}(x)\right|^{3}, \ldots,\left|\psi^{\prime}(x)\right|^{2(k-\ell+1)-1}\right)}{\psi^{\prime}(x)^{2 k-1}}\right| \\
& \quad \leq C^{\prime}\left|\psi^{\prime}(x)^{2 k-1}\right|
\end{aligned}
$$

with constants $C, C^{\prime}>0$.

We go back to the derivatives of $h \circ \psi$ in (3.20) and bound them individually. For $k=0$, we simply estimate

$$
\begin{aligned}
\|h \circ \psi\|_{L_{2}(\mathbb{T})} & =\left(\int_{-\frac{1}{2}}^{\frac{1}{2}}\left|h(\psi(x)) \psi^{\prime}(x)^{-\frac{1}{2}}\right|^{2} \psi^{\prime}(x) \mathrm{d} x\right)^{\frac{1}{2}} \\
& \leq\left\|\psi^{\prime}(\circ)^{-\frac{1}{2}}\right\|_{L_{\infty}(\mathbb{T})}\left(\int_{-\infty}^{\infty}|h(y)|^{2} \mathrm{~d} y\right)^{\frac{1}{2}},
\end{aligned}
$$

which exists if $\psi^{\prime}(\circ)^{-\frac{1}{2}} \in L_{\infty}(\mathbb{T})$. With the Faá di Bruno formula (3.20) and the upper bound (3.23) for $\frac{\mathrm{d}^{k}}{\mathrm{~d} x^{k}}[h \circ \psi](x)$, we obtain

$$
\begin{aligned}
\left\|\frac{\mathrm{d}^{k}}{\mathrm{~d} x^{k}}[h \circ \psi](x)\right\|_{L_{2}(\mathbb{T})} & =\left(\int_{-\frac{1}{2}}^{\frac{1}{2}}\left|\frac{\mathrm{d}^{k}}{\mathrm{~d} x^{k}}[h \circ \psi](x) \psi^{\prime}(x)^{-\frac{1}{2}}\right|^{2} \psi^{\prime}(x) \mathrm{d} x\right)^{\frac{1}{2}} \\
& \leq\left\|\psi^{\prime}(x)^{2 k-\frac{3}{2}}\right\|_{L_{\infty}(\mathbb{T})}\left(\int_{-\frac{1}{2}}^{\frac{1}{2}}\left|\sum_{j=1}^{k} h^{(j)}(\psi(x))\right|^{2} \psi^{\prime}(x) \mathrm{d} x\right)^{\frac{1}{2}} \\
& \leq C \cdot\left\|\psi^{\prime}(x)^{2 k-\frac{3}{2}}\right\|_{L_{\infty}(\mathbb{T})} \sum_{j=1}^{k}\left\|\frac{\mathrm{d}^{j}}{\mathrm{~d} y^{j}}[h(y)]\right\|_{L_{2}(\mathbb{R})} .
\end{aligned}
$$


By inserting (3.24) and (3.25) into (3.19), we in total have, for all $\ell \in \mathbb{N}_{0}$, the estimate

$$
\begin{aligned}
& \left\|\frac{\mathrm{d}^{\ell}}{\mathrm{d} x^{\ell}}[f](x)\right\|_{L_{2}(\mathbb{T})} \\
& \leq \sum_{k=0}^{\ell}\left(\begin{array}{l}
\ell \\
k
\end{array}\right)\left\|\frac{\mathrm{d}^{k}}{\mathrm{~d} x^{k}}[h \circ \psi](x) \frac{\mathrm{d}^{\ell-k}}{\mathrm{~d} x^{\ell-k}}\left[\sqrt{(\omega \circ \psi) \psi^{\prime}}\right](x)\right\|_{L_{2}(\mathbb{T})} \\
& \leq\left\|\frac{\mathrm{d}^{\ell}}{\mathrm{d} x^{\ell}}\left[\sqrt{(\omega \circ \psi) \psi^{\prime}}\right](x) \psi^{\prime}(x)^{-\frac{1}{2}}\right\|\left\|_{L_{\infty}(\mathbb{T})}\right\| h \|_{L_{2}(\mathbb{R})} \\
& \quad+C \cdot \sum_{k=1}^{\ell}\left(\begin{array}{l}
\ell \\
k
\end{array}\right)\left\|\frac{\mathrm{d}^{\ell-k}}{\mathrm{~d} x^{\ell-k}}\left[\sqrt{(\omega \circ \psi) \psi^{\prime}}\right](x) \psi^{\prime}(x)^{2 k-\frac{3}{2}}\right\|_{L_{\infty}(\mathbb{T})} \sum_{j=1}^{k}\left\|\frac{\mathrm{d}^{j}}{\mathrm{~d} y^{j}}[h(y)]\right\|_{L_{2}(\mathbb{R})} \\
& \leq C^{\prime} \max _{k=0, \ldots, \ell}\left\|\frac{\mathrm{d}^{\ell-k}}{\mathrm{~d} x^{\ell-k}}\left[\sqrt{(\omega \circ \psi) \psi^{\prime}}\right](x) \psi^{\prime}(x)^{\max \left(-\frac{1}{2}, 2 k-\frac{3}{2}\right)}\right\|_{L_{\infty}(\mathbb{T})} \times \\
& \quad \times\left(\|h\|_{L_{2}(\mathbb{R})}+\sum_{k=1}^{\ell}\|h\|_{H^{k}(\mathbb{R})}\right) \\
& \leq C^{\prime} \max _{k=0, \ldots, \ell}\left\|\frac{\mathrm{d}^{\ell-k}}{\mathrm{~d} x^{\ell-k}}\left[\sqrt{(\omega \circ \psi) \psi^{\prime}}\right](x) \psi^{\prime}(x)^{\max \left(-\frac{1}{2}, 2 k-\frac{3}{2}\right)}\right\|_{L_{\infty}(\mathbb{T})}(\ell+1)\|h\|_{H^{\ell}(\mathbb{R})}
\end{aligned}
$$

with constants $C, C^{\prime} \geq 1$. This upper bound is finite as long as the $L_{\infty}$-norms are finite and $h \in H^{m}(\mathbb{R})$. In total we finally obtain the estimate

$$
\begin{aligned}
& \|f\|_{H^{m}(\mathbb{T})}=\left(\sum_{\ell=0}^{m}\left\|\frac{\mathrm{d}^{\ell}}{\mathrm{d} x^{\ell}}[f](x)\right\|_{L_{2}(\mathbb{T})}^{2}\right)^{\frac{1}{2}} \\
& \leq C \max _{\ell=0, \ldots, m}\left(\max _{k=0, \ldots, \ell}\left\|\frac{\mathrm{d}^{\ell-k}}{\mathrm{~d} x^{\ell-k}}\left[\sqrt{(\omega \circ \psi) \psi^{\prime}}\right](x) \psi^{\prime}(x)^{\max \left(-\frac{1}{2}, 2 k-\frac{3}{2}\right)}\right\|_{L_{\infty}(\mathbb{T})}\right) \\
& \quad \times\left(\sum_{\ell=0}^{m}(\ell+1)^{2}\|h\|_{H^{\ell}(\mathbb{R})}^{2}\right)^{\frac{1}{2}} \\
& \leq C \max _{\ell=0, \ldots, m}\left(\max _{k=0, \ldots, \ell}\left\|\frac{\mathrm{d}^{\ell-k}}{\mathrm{~d} x^{\ell-k}}\left[\sqrt{(\omega \circ \psi) \psi^{\prime}}\right](x) \psi^{\prime}(x)^{\max \left(-\frac{1}{2}, 2 k-\frac{3}{2}\right)}\right\|_{L_{\infty}(\mathbb{T})}\right) \\
& \quad \times(m+1)^{\frac{3}{2}}\|h\|_{H^{m}(\mathbb{R})} .
\end{aligned}
$$

Next, we generalize the previous theorem by proving its multivariate version. Again, to simplify the notation in (1.6) of the $d$-variate differential operator $D^{\mathbf{m}}[f](\mathbf{x})$ with both $\mathbf{m}=\left(m_{1}, \ldots, m_{d}\right) \in \mathbb{N}_{0}^{d}$ and $\mathbf{x}=\left(x_{1}, \ldots, x_{d}\right)^{\top} \in \mathbb{R}^{d}$, we use equivalent expressions for certain (partial) derivatives and state explicit arguments in the various norms. When differentiating a multivariate function $f$ with respect to the $j$-th coordinate $m_{j}$-times, we write

$$
\partial^{m_{j}}[f](\mathbf{x}):=\frac{\partial^{m_{j}}}{\partial x_{j}^{m_{j}}}[f](\mathbf{x})=D^{\left(0, \ldots, 0, m_{j}, 0, \ldots, 0\right)}[f](\mathbf{x}) .
$$


For the first and $\ell$-th derivatives of univariate functions with $\ell \in \mathbb{N}$, we use the notation

$$
\psi_{j}^{\prime}\left(x_{j}\right):=\frac{\mathrm{d}}{\mathrm{d} x_{j}}\left[\psi_{j}\right]\left(x_{j}\right) \quad \text { and } \quad \psi_{j}^{(\ell)}\left(x_{j}\right):=\frac{\mathrm{d}^{\ell}}{\mathrm{d} x_{j}^{\ell}}\left[\psi_{j}\right]\left(x_{j}\right) .
$$

Similar to (3.17) we consider multivariate transformed functions $f \in L_{2}\left(\mathbb{T}^{d}\right)$ of the form

$$
\begin{aligned}
f(\mathbf{x}) & =(h \circ \psi)(\mathbf{x}) \sqrt{(\omega \circ \psi)(\mathbf{x}) D^{\mathbf{1}}[\psi](\mathbf{x})} \\
& =h\left(\psi_{1}\left(x_{1}\right), \ldots, \psi_{d}\left(x_{d}\right)\right) \prod_{k=1}^{d} \sqrt{\omega_{k}\left(\psi_{k}\left(x_{k}\right)\right) \psi_{k}^{\prime}\left(x_{k}\right)}, \quad \mathbf{x} \in \mathbb{T}^{d},
\end{aligned}
$$

which are the result of applying the multivariate change of variables

$$
\mathbf{y}=\left(y_{1}, \ldots, y_{d}\right)^{\top}=\left(\psi_{1}\left(x_{1}\right), \ldots, \psi_{d}\left(x_{d}\right)\right)^{\top}=\psi(\mathbf{x})
$$

defined in (3.4) to a function $h \in L_{2}\left(\mathbb{R}^{d}, \omega\right)$ with a product weight $\omega$ as in (3.10). For this, we have the identity

$$
\begin{aligned}
\|h\|_{L_{2}\left(\mathbb{R}^{d}, \omega\right)}^{2} & =\int_{\mathbb{R}^{d}}|h(\mathbf{y})|^{2} \omega(\mathbf{y}) \mathrm{d} \mathbf{y} \\
& =\int_{\mathbb{T}^{d}}|(h \circ \psi)(\mathbf{x})|^{2}(\omega \circ \psi)(\mathbf{x}) D^{\mathbf{1}}[\psi](\mathbf{x}) \mathrm{d} \mathbf{x}=\|f\|_{L_{2}\left(\mathbb{T}^{d}\right)}^{2} .
\end{aligned}
$$

Again, we derive a set of sufficient $L_{\infty}$-conditions for the multivariate transformation $\psi$ and the product weight $\omega$ that determine when a function $h \in L_{2}\left(\mathbb{R}^{d}, \omega\right) \cap H_{\text {mix }}^{m}\left(\mathbb{R}^{d}\right)$ can be transformed by $\psi$ into an $f \in H_{\text {mix }}^{m}\left(\mathbb{T}^{d}\right)$ of the form (3.27).

THEOREM 3.5. Let be given the dimension $d \in \mathbb{N}, m \in \mathbb{N}_{0}$, a d-variate transformation $\psi:\left(-\frac{1}{2}, \frac{1}{2}\right)^{d} \rightarrow \mathbb{R}^{d}$ as defined in (3.4) with the $d$-variate density function $\varrho(\mathbf{y})=\prod_{j=1}^{d} \varrho_{j}\left(y_{j}\right)$ of $\psi$ as in (3.3), a non-negative product weight function $\omega: \mathbb{R}^{d} \rightarrow[0, \infty)$ as in (3.10), a multivariate function $h \in L_{2}\left(\mathbb{R}^{d}, \omega\right) \cap H_{\text {mix }}^{m}\left(\mathbb{R}^{d}\right)$, and the corresponding transformed functions $f$ of the form (3.27).

If for all multi-indices $\mathbf{m}=\left(m_{1}, \ldots, m_{d}\right)^{\top} \in \mathbb{N}_{0}^{d},\|\mathbf{m}\|_{\ell_{\infty}} \leq m$, and all $j_{\ell}=0, \ldots, m$, $\ell=1, \ldots, d$, we have

$$
\text { and } \max _{j_{\ell}=0, \ldots, m_{\ell}}\left\|\partial^{m_{\ell}-j_{\ell}}\left[\sqrt{\left(\omega_{\ell} \circ \psi_{\ell}\right) \psi_{\ell}^{\prime}}\right]\left(x_{\ell}\right) \psi_{\ell}^{\prime}\left(x_{\ell}\right)^{\max \left(-\frac{1}{2}, 2 j_{\ell}-\frac{3}{2}\right)}\right\|_{L_{\infty}(\mathbb{T})}<\infty
$$

$$
\partial^{j_{\ell}}[\varrho]\left(y_{\ell}\right) \in \mathcal{C}_{0}(\mathbb{R}), \quad \partial^{j_{\ell}}[\psi]\left(x_{\ell}\right) \in \mathcal{C}((-1 / 2,1 / 2))
$$

then $f \in H_{\text {mix }}^{m}\left(\mathbb{T}^{d}\right)$.

Proof. For $h \in L_{2}\left(\mathbb{R}^{d}, \omega\right) \cap H_{\text {mix }}^{m}\left(\mathbb{R}^{d}\right)$ with $m \in \mathbb{N}_{0}$ and a transformation $\psi$ as defined in (3.4), we consider the function $f$ given in (3.27). In order to prove that $f \in H_{\text {mix }}^{m}\left(\mathbb{T}^{d}\right)$, we have to show that for all multi-indices $\mathbf{m}=\left(m_{1}, \ldots, m_{d}\right)^{\top} \in \mathbb{N}_{0}^{d}$ with $\|\mathbf{m}\|_{\ell_{\infty}} \leq m$, we have $\left\|D^{\mathbf{m}}[f](\mathbf{x})\right\|_{L_{2}\left(\mathbb{T}^{d}\right)}<\infty$.

Let $\mathbf{m}=\left(m_{1}, \ldots, m_{d}\right)^{\top} \in \mathbb{N}_{0}^{d}$ be any multi-index with $\|\mathbf{m}\|_{\ell_{\infty}} \leq m$. For a multivariate transformed function of the form (3.27) we have

$$
\left\|D^{\mathbf{m}}[f](\mathbf{x})\right\|_{L_{2}\left(\mathbb{T}^{d}\right)}=\left(\int_{\mathbb{T}^{d}}\left|D^{\mathbf{m}}\left[(h \circ \psi) \prod_{k=1}^{d} \sqrt{\left(\omega_{k} \circ \psi_{k}\right) \psi_{k}^{\prime}}\right](\mathbf{x})\right|^{2} \mathrm{~d} \mathbf{x}\right)^{\frac{1}{2}} .
$$


The product weight function in the transformed function $f$ in (3.27) yields

$$
\begin{aligned}
D^{\mathbf{m}} & {\left[(h \circ \psi) \prod_{k=1}^{d} \sqrt{\left(\omega_{k} \circ \psi_{k}\right) \psi_{k}^{\prime}}\right](\mathbf{x}) } \\
& =\partial^{m_{d}}\left[\ldots \partial^{m_{2}}\left[\partial^{m_{1}}\left[(h \circ \psi) \prod_{k=1}^{d} \sqrt{\left(\omega_{k} \circ \psi_{k}\right) \psi_{k}^{\prime}}\right]\left(x_{1}\right)\right]\left(x_{2}\right) \ldots\right]\left(x_{d}\right) .
\end{aligned}
$$

By applying the Leibniz formula as in (3.18) we obtain, for all $\ell=1, \ldots, d$,

$$
\begin{aligned}
\partial^{m_{\ell}} & {\left[(h \circ \psi) \prod_{k=1}^{d} \sqrt{\left(\omega_{k} \circ \psi_{k}\right) \psi_{k}^{\prime}}\right]\left(x_{\ell}\right) } \\
& =\sum_{j_{\ell}=0}^{m_{\ell}}\left(\begin{array}{c}
m_{\ell} \\
j_{\ell}
\end{array}\right) \partial^{j_{\ell}}[h \circ \psi]\left(x_{\ell}\right) \partial^{m_{\ell}-j_{\ell}}\left[\prod_{k=1}^{d} \sqrt{\left(\omega_{k} \circ \psi_{k}\right) \psi_{k}^{\prime}}\right]\left(x_{\ell}\right),
\end{aligned}
$$

and in total we rewrite the expression in (3.30) as

$$
\begin{aligned}
D^{\mathbf{m}}\left[(h \circ \psi) \prod_{k=1}^{d} \sqrt{\left(\omega_{k} \circ \psi_{k}\right) \psi_{k}^{\prime}}\right](\mathbf{x}) \\
=\sum_{j_{1}=0}^{m_{1}}\left(\begin{array}{c}
m_{1} \\
j_{1}
\end{array}\right) \ldots \sum_{j_{d}=0}^{m_{d}}\left(\begin{array}{c}
m_{d} \\
j_{d}
\end{array}\right) D^{\left(j_{1}, \ldots, j_{d}\right)}[h \circ \psi](\mathbf{x}) \\
\quad \times D^{\left(m_{1}-j_{1}, \ldots, m_{d}-j_{d}\right)}\left[\prod_{k=1}^{d} \sqrt{\left(\omega_{k} \circ \psi_{k}\right) \psi_{k}^{\prime}}\right](\mathbf{x}) .
\end{aligned}
$$

Next, we apply the Faá di Bruno formula (3.20) to each univariate $j_{k}$-th derivative of $h \circ \psi$ in (3.31) so that for $\ell=1, \ldots, d$ we have

$$
\partial^{j_{\ell}}[h \circ \psi](\mathbf{x})= \begin{cases}\sum_{i_{\ell}=1}^{j_{\ell}} \partial^{i_{\ell}}[h](\psi(\mathbf{x})) B_{j_{\ell}, i_{\ell}}\left(\psi_{\ell}^{\prime}\left(x_{\ell}\right), \ldots, \psi_{\ell}^{\left(j_{\ell}-i_{\ell}+1\right)}\left(x_{\ell}\right)\right) & j_{\ell} \in \mathbb{N}, \\ h(\psi(\mathbf{x})) & j_{\ell}=0,\end{cases}
$$

i.e., $B_{0, i_{\ell}}\left(\psi_{\ell}^{\prime}\left(x_{\ell}\right), \psi_{\ell}^{\prime \prime}\left(x_{\ell}\right), \ldots, \psi_{\ell}^{\left(j_{\ell}-i_{\ell}+1\right)}\left(x_{\ell}\right)\right)=1$.

We combine the norm $\left\|D^{\mathbf{m}}[f](\mathbf{x})\right\|_{L_{2}\left(\mathbb{T}^{d}\right)}$ in (3.29) with the expression resulting from applying the Leibniz formula to $D^{\mathbf{m}}[f]$ in (3.32) and the subsequent application of the Faá di Bruno formula in (3.33). Then we estimate

$$
\begin{aligned}
\left\|D^{\mathbf{m}}[f](\mathbf{x})\right\|_{L_{2}\left(\mathbb{T}^{d}\right)} \leq & \sum_{j_{1}=0, \ldots, j_{d}=0}^{m_{1}, \ldots, m_{d}} \prod_{\ell=1}^{d}\left(\begin{array}{c}
m_{\ell} \\
j_{\ell}
\end{array}\right) \sum_{i_{1}=1, \ldots, i_{d}=1}^{j_{1}, \ldots, j_{d}}\left(\int_{\mathbb{T}^{d}}\left|D^{\left(i_{1}, \ldots, i_{d}\right)}[h](\psi(\mathbf{x}))\right|^{2}\right. \\
& \times \prod_{\ell=1}^{d}\left|B_{j_{\ell}, i_{\ell}}\left(\psi_{\ell}^{\prime}\left(x_{\ell}\right), \ldots, \psi_{\ell}^{\left(j_{\ell}-i_{\ell}+1\right)}\left(x_{\ell}\right)\right)\right|^{2} \\
& \left.\times\left|D^{\left(m_{1}-j_{1}, \ldots, m_{d}-j_{d}\right)}\left[\prod_{k=1}^{d} \sqrt{\left(\omega_{k} \circ \psi_{k}\right) \psi_{k}^{\prime}}\right](\mathbf{x})\right|^{2} \mathrm{~d} \mathbf{x}\right)^{\frac{1}{2}}
\end{aligned}
$$


In the multivariate integral appearing in (3.34) we bound each coordinate separately with the univariate arguments of the previous proof by fixing all but one coordinate one after another. Recalling the arguments in (3.23), if all appearing derivatives of $\psi_{\ell}$ are in $\mathcal{C}(-1 / 2,1 / 2)$ and the corresponding derivatives of the density $\varrho_{\ell}$ are in $\mathcal{C}_{0}(\mathbb{R})$, then for all Bell polynomials $B_{j_{\ell}, i_{\ell}}$ with $j_{\ell} \geq 1$ appearing in (3.33) and (3.34), there is some constant $C>0$ so that we can estimate

$$
\left|B_{j_{\ell}, i_{\ell}}\left(\psi_{\ell}^{\prime}\left(x_{\ell}\right), \psi_{\ell}^{\prime \prime}\left(x_{\ell}\right), \ldots, \psi_{\ell}^{\left(j_{\ell}-i_{\ell}+1\right)}\left(x_{\ell}\right)\right)\right| \leq C\left|\psi_{\ell}^{\prime}\left(x_{\ell}\right)\right|^{2 j_{\ell}-1} .
$$

Analogously to (3.24) and (3.25), for each $\ell=1, \ldots, d$, we have to separate the summand for $j_{\ell}=0$ from the summands corresponding to $j_{\ell}=1, \ldots, d$. Starting with $\ell=1$ we bound (3.34) as in (3.26) after inserting the productive one $1=\psi_{1}^{\prime}\left(x_{1}\right) \frac{1}{\psi_{1}^{\prime}\left(x_{1}\right)}$, so that

$$
\begin{aligned}
& \left\|D^{\mathbf{m}}[f](\mathbf{x})\right\|_{L_{2}\left(\mathbb{T}^{d}\right)} \\
& \leq C_{1}\left(\begin{array}{c}
m_{1} \\
\left\lceil\frac{m_{1}}{2}\right\rceil
\end{array}\right) \max _{j_{1}=0, \ldots, m_{1}}\left\|\partial^{m_{1}-j_{1}}\left[\sqrt{\left(\omega_{1} \circ \psi_{1}\right) \psi_{1}^{\prime}}\right]\left(x_{1}\right) \psi_{1}^{\prime}\left(x_{1}\right)^{\max \left(-\frac{1}{2}, 2 j_{1}-\frac{3}{2}\right)}\right\|_{L_{\infty}(\mathbb{T})} \\
& \times \sum_{j_{2}=0, \ldots, j_{d}=0}^{m_{2}, \ldots, m_{d}} \prod_{\ell=2}^{d}\left(\begin{array}{c}
m_{\ell} \\
j_{\ell}
\end{array}\right) \sum_{i_{2}=1, \ldots, i_{d}=1}^{j_{2}, \ldots, j_{d}}\left(\int_{\mathbb{T}^{d-1}}\right. \\
& \int_{\mathbb{T}}\left|D^{\left(i_{1}, \ldots, i_{d}\right)}[h]\left(\psi_{1}\left(x_{1}\right), \ldots, \psi_{d}\left(x_{d}\right)\right)\right|^{2} \psi_{1}^{\prime}\left(x_{1}\right) \mathrm{d} x_{1} \\
& \times \prod_{\ell=2}^{d}\left|B_{j_{\ell}, i_{\ell}}\left(\psi_{\ell}^{\prime}\left(x_{\ell}\right), \ldots, \psi_{\ell}^{\left(j_{\ell}-i_{\ell}+1\right)}\left(x_{\ell}\right)\right)\right|^{2} \\
& \times\left|D^{\left(m_{2}-j_{2}, \ldots, m_{d}-j_{d}\right)}\left[\prod_{k=2}^{d} \sqrt{\left(\omega_{k} \circ \psi_{k}\right) \psi_{k}^{\prime}}\right]\left(x_{2}, \ldots, x_{d}\right)\right|^{2} \\
& \left.\mathrm{~d}\left(x_{2}, \ldots, x_{d}\right)\right)^{\frac{1}{2}}
\end{aligned}
$$

After repeating this process for $\ell=2, \ldots, d$ and inserting the inverse transformations $x_{\ell}=\psi_{\ell}^{-1}\left(y_{\ell}\right)$ for all $\ell=1, \ldots, d$, we end up with the estimate

$$
\begin{aligned}
& \left\|D^{\mathbf{m}}[f](\mathbf{x})\right\|_{L_{2}\left(\mathbb{T}^{d}\right)} \\
& \leq \prod_{\ell=1}^{d} C_{\ell}\left(\begin{array}{c}
m_{\ell} \\
\left\lceil\frac{m_{\ell}}{2}\right\rceil
\end{array}\right) \max _{j_{\ell}=0, \ldots, m_{\ell}}\left\|\partial^{m_{\ell}-j_{\ell}}\left[\sqrt{\left(\omega_{\ell} \circ \psi_{\ell}\right) \psi_{\ell}^{\prime}}\right]\left(x_{\ell}\right) \psi_{\ell}^{\prime}\left(x_{\ell}\right)^{\max \left(-\frac{1}{2}, 2 j_{\ell}-\frac{3}{2}\right)}\right\|_{L_{\infty}(\mathbb{T})} \\
& \quad \times \sum_{j_{1}=0, \ldots, j_{d}=0}^{m_{1}, \ldots, m_{d}} \sum_{i_{1}=1, \ldots, i_{d}=1}^{j_{1}, \ldots, j_{d}}\left(\int_{\mathbb{T}^{d}}\left|D^{\left(i_{1}, \ldots, i_{d}\right)}[h]\left(\psi_{1}\left(x_{1}\right), \ldots, \psi_{d}\left(x_{d}\right)\right)\right|^{2} \prod_{\ell=1}^{d} \psi_{\ell}^{\prime}\left(x_{\ell}\right) \mathrm{d} \mathbf{x}\right)^{\frac{1}{2}} \\
& \quad \prod_{\ell=1}^{d} \max _{j_{\ell}=0, \ldots, m_{\ell}}\left\|\partial^{m_{\ell}-j_{\ell}}\left[\sqrt{\left(\omega_{\ell} \circ \psi_{\ell}\right) \psi_{\ell}^{\prime}}\right]\left(x_{\ell}\right) \psi_{\ell}^{\prime}\left(x_{\ell}\right)^{\max \left(-\frac{1}{2}, 2 j_{\ell}-\frac{3}{2}\right)}\right\|_{L_{\infty}(\mathbb{T})} \\
& \quad \sum_{j_{1}=0, \ldots, j_{d}=0}^{m_{1}, \ldots, m_{d}} \sum_{i_{1}=1, \ldots, i_{d}=1}^{j_{1}, \ldots, j_{d}}\left(\int_{\mathbb{R}^{d}}\left|D^{\left(i_{1}, \ldots, i_{d}\right)}[h]\left(y_{1}, \ldots, y_{d}\right)\right|^{2} \mathrm{~d} \mathbf{y}\right)^{\frac{1}{2}} \\
& \leq C \prod_{\ell=1}^{d} \max _{j_{\ell}=0, \ldots, m_{\ell}}\left\|\partial^{m_{\ell}-j_{\ell}}\left[\sqrt{\left(\omega_{\ell} \circ \psi_{\ell}\right) \psi_{\ell}^{\prime}}\right]\left(x_{\ell}\right) \psi_{\ell}^{\prime}\left(x_{\ell}\right)^{\max \left(-\frac{1}{2}, 2 j_{\ell}-\frac{3}{2}\right)}\right\|_{L_{\infty}(\mathbb{T})}
\end{aligned}
$$




$$
\times \sum_{j_{1}=0, \ldots, j_{d}=0}^{m_{1}, \ldots, m_{d}}\|h\|_{H_{\text {mix }}^{j}\left(\mathbb{R}^{d}\right)},
$$

with $j=\max \left(j_{1}, \ldots, j_{d}\right)$ in the last inequality.

Since the previous estimate is valid for all multi-indices $\mathbf{m}=\left(m_{1}, \ldots, m_{d}\right)^{\top} \in \mathbb{N}_{0}^{d}$ with $\|\mathbf{m}\|_{\ell_{\infty}} \leq m$, we finally obtain the bound

$$
\begin{aligned}
& \|f\|_{H_{\text {mix }}^{m}\left(\mathbb{T}^{d}\right)} \\
= & \left(\sum_{\|\mathbf{m}\|_{\ell_{\infty} \leq m} \leq}\left\|D^{\mathbf{m}}[f](\mathbf{x})\right\|_{L_{2}\left(\mathbb{T}^{d}\right)}^{2}\right)^{\frac{1}{2}}=\left(\sum_{m_{1}=0, \ldots, m_{d}=0}^{m, \ldots, m}\left\|D^{\mathbf{m}}[f](\mathbf{x})\right\|_{L_{2}\left(\mathbb{T}^{d}\right)}^{2}\right)^{\frac{1}{2}} \\
\leq & C \prod_{\ell=1}^{d} \max _{m_{\ell}=0, \ldots, m} \\
& \left(\max _{j_{\ell}=0, \ldots, m_{\ell}}\left\|\partial^{m_{\ell}-j_{\ell}}\left[\sqrt{\left(\omega_{\ell} \circ \psi_{\ell}\right) \psi_{\ell}^{\prime}}\right]\left(x_{\ell}\right) \psi_{\ell}^{\prime}\left(x_{\ell}\right)^{\max \left(-\frac{1}{2}, 2 j_{\ell}-\frac{3}{2}\right)}\right\|_{L_{\infty}(\mathbb{T})}\right) \\
& \times(m+1)^{d}\|h\|_{H_{\text {mix }}^{m}\left(\mathbb{R}^{d}\right)} .
\end{aligned}
$$

3.5. Approximation of transformed functions. We establish two specific approximation error bounds for functions defined on $\mathbb{R}^{d}$ based on the approximation error bounds on the torus $\mathbb{T}^{d}$ that we recalled in Theorems 2.2 and 2.3. The corresponding proofs rely heavily on the previously introduced sufficient conditions in Theorem 3.5 that describe when Sobolev functions $h \in L_{2}\left(\mathbb{R}^{d}, \omega\right) \cap H_{\text {mix }}^{m}\left(\mathbb{R}^{d}\right)$ with a multivariate weight function $\omega: \mathbb{R}^{d} \rightarrow[0, \infty)$ as given in (3.10) can be transformed into Sobolev functions of dominated mixed smoothness on $\mathbb{T}^{d}$ of the form (3.27) by multivariate transformations $\psi:\left(-\frac{1}{2}, \frac{1}{2}\right)^{d} \rightarrow \mathbb{R}^{d}$ as in (3.4).

At first, we fix some notation for certain multivariate objects. Based on the definition of a rank-1 lattice $\Lambda(\mathbf{z}, M)$ in (2.5), we define a transformed rank-1 lattice as

$$
\Lambda_{\psi}(\mathbf{z}, M):=\left\{\mathbf{y}_{j}:=\psi\left(\mathbf{x}_{j}\right): \mathbf{x}_{j} \in \Lambda(\mathbf{z}, M), j=0, \ldots, M-1\right\} .
$$

Accordingly, we denote the transformed reconstructing rank-1 lattice by $\Lambda_{\psi}(\mathbf{z}, M, I)$.

Besides the weight function $\omega$, also the density $\varrho$ of the transformation $\psi$ is of product form as defined in (3.3), i.e., it is the product of univariate densities $\varrho_{j}\left(y_{j}\right), j=1, \ldots, d$. Hence, based on the functions $\varphi_{k}$ in (3.11) this product form extends to

$$
\varphi_{\mathbf{k}}(\mathbf{y}):=\prod_{j=1}^{d} \varphi_{k_{j}}\left(y_{j}\right) .
$$

Similar to (3.12), the multivariate weighted $L_{2}\left(\mathbb{R}^{d}, \omega\right)$ scalar product reads as

$$
\left(h_{1}, h_{2}\right)_{L_{2}\left(\mathbb{R}^{d}, \omega\right)}:=\int_{\mathbb{R}^{d}} \prod_{j=1}^{d} \omega_{j}\left(y_{j}\right) h_{1}(\mathbf{y}) \overline{h_{2}(\mathbf{y})} \mathrm{d} \mathbf{y},
$$

and similar to (3.13), the multivariate Fourier coefficients are naturally given with respect to this scalar product as

$$
\hat{h}_{\mathbf{k}}=\left(h, \varphi_{\mathbf{k}}\right)_{L_{2}\left(\mathbb{R}^{d}, \omega\right)} .
$$


As before in (3.14), we define the multivariate Fourier partial sum as

$$
S_{I} h(\mathbf{y}):=\sum_{\mathbf{k} \in I} \hat{h}_{\mathbf{k}} \varphi_{\mathbf{k}}(\mathbf{y}) .
$$

Let $f \in L_{2}\left(\mathbb{T}^{d}\right)$. Then for each $I \subset \mathbb{Z}^{d}$ the system $\left\{\varphi_{\mathbf{k}}\right\}_{\mathbf{k} \in I}$ spans the space of transformed trigonometric polynomials

$$
\Pi_{I, \psi}:=\operatorname{span}\left\{\sqrt{\frac{\varrho(0)}{\omega(\circ)}} \mathrm{e}^{2 \pi \mathbf{i k} \cdot \psi^{-1}(\circ)}: \mathbf{k} \in I\right\} .
$$

Similar to (2.7), for transformed trigonometric polynomials $h \in \Pi_{I, \psi}$, transformed lattice nodes $\mathbf{y}_{j} \in \Lambda_{\psi}(\mathbf{z}, M, I)$, and $\mathbf{k} \in I$, we have the exact integration property of the form

$$
\begin{aligned}
\hat{h}_{\mathbf{k}} & =\int_{\mathbb{R}^{d}} h(\mathbf{y}) \sqrt{\varrho(\mathbf{y}) \omega(\mathbf{y})} \mathrm{e}^{-2 \pi \mathrm{i} \mathbf{k} \cdot \psi^{-1}(\mathbf{y})} \mathrm{d} y=\int_{\mathbb{T}^{d}} f(\mathbf{x}) \mathrm{e}^{-2 \pi \mathrm{i} \mathbf{k} \cdot \mathbf{x}} \mathrm{d} \mathbf{x} \\
& =\frac{1}{M} \sum_{j=0}^{M-1} f\left(\mathbf{x}_{j}\right) \mathrm{e}^{-2 \pi \mathbf{i} \cdot \mathbf{x}_{j}}=\frac{1}{M} \sum_{j=0}^{M-1} h\left(\mathbf{y}_{j}\right) \sqrt{\frac{\varrho\left(\mathbf{y}_{j}\right)}{\omega\left(\mathbf{y}_{j}\right)}} \mathrm{e}^{-2 \pi \mathbf{i} \mathbf{k} \cdot \psi^{-1}\left(\mathbf{y}_{j}\right)}=\hat{h}_{\mathbf{k}}^{\Lambda}
\end{aligned}
$$

Generally, the multivariate approximated Fourier coefficients of the form

$$
\hat{h}_{\mathbf{k}}^{\Lambda}=\frac{1}{M} \sum_{j=0}^{M-1} h\left(\mathbf{y}_{j}\right) \sqrt{\frac{\varrho\left(\mathbf{y}_{j}\right)}{\omega\left(\mathbf{y}_{j}\right)}} \mathrm{e}^{-2 \pi \mathbf{i k} \cdot \psi^{-1}\left(\mathbf{y}_{j}\right)}=\frac{1}{M} \sum_{j=0}^{M-1} h\left(\mathbf{y}_{j}\right) \overline{\varphi_{\mathbf{k}}\left(\mathbf{y}_{j}\right)}
$$

approximate the multivariate Fourier coefficients $\hat{h}_{\mathbf{k}}$. Finally, the multivariate version of the approximated Fourier partial sum is given by

$$
S_{I}^{\Lambda} h(\mathbf{x}):=\sum_{\mathbf{k} \in I} \hat{h}_{\mathbf{k}}^{\Lambda} \varphi_{\mathbf{k}}(\mathbf{y})
$$

Similarly to the $\mathcal{H}^{\beta}\left(\mathbb{T}^{d}\right)$-norm in (1.2), we define a norm of weighted Fourier coefficients $\hat{h}_{\mathbf{k}}$ of the form

$$
\|h\|_{\mathcal{H}^{m}\left(\mathbb{R}^{d}\right)}^{2}:=\sum_{\mathbf{k} \in \mathbb{Z}^{d}} \omega_{\mathrm{hc}}(\mathbf{k})^{2 m}\left|\hat{h}_{\mathbf{k}}\right|^{2}
$$

With these rewritten objects we transfer the approximation error bounds in Theorems 2.2 and 2.3 for functions defined on the torus to $\mathbb{R}^{d}$.

3.5.1. $\boldsymbol{L}_{\infty}$-approximation error. Based on the $L_{\infty}\left(\mathbb{T}^{d}\right)$-approximation error bound (2.8) and the conditions proposed in Theorem 3.5, we prove a similar upper bound for the approximation error $\left\|h-S_{I_{N}^{d}}^{\Lambda} h\right\|$ in terms of a weighted $L_{\infty}$-norm on $\mathbb{R}^{d}$.

THEOREM 3.6. Let be given $d \in \mathbb{N}, m \in \mathbb{N}_{0}$, a hyperbolic cross $I_{N}^{d}$ with $N \geq 2^{d+1}$, and a reconstructing rank-1 lattice $\Lambda\left(\mathbf{z}, M, I_{N}^{d}\right)$. Let $\psi$ be a multivariate transformation as defined in (3.4) with its corresponding density function @ in product form (3.3). Let $\omega$ be a weight function as in (3.10), and consider a multivariate function $h \in L_{2}\left(\mathbb{R}^{d}, \omega\right) \cap H_{\mathrm{mix}}^{m}\left(\mathbb{R}^{d}\right)$. Let $\lambda>\frac{1}{2}$. Furthermore, for all multi-indices $\mathbf{m}=\left(m_{1}, \ldots, m_{d}\right)^{\top} \in \mathbb{N}_{0}^{d}$ with $\|\mathbf{m}\|_{\ell_{\infty}} \leq m$ and all $j_{\ell}=0, \ldots, m, \ell=1, \ldots, d$, we assume

$$
\partial^{j_{\ell}}[\varrho]\left(y_{\ell}\right) \in \mathcal{C}_{0}(\mathbb{R}), \quad \partial^{j_{\ell}}[\psi]\left(x_{\ell}\right) \in \mathcal{C}((-1 / 2,1 / 2))
$$


and

$$
\max _{j_{\ell}=0, \ldots, m_{\ell}}\left\|\partial^{m_{\ell}-j_{\ell}}\left[\sqrt{\left(\omega_{\ell} \circ \psi_{\ell}\right) \psi_{\ell}^{\prime}}\right]\left(x_{\ell}\right) \psi_{\ell}^{\prime}\left(x_{\ell}\right)^{\max \left(-\frac{1}{2}, 2 j_{\ell}-\frac{3}{2}\right)}\right\|_{L_{\infty}(\mathbb{T})}<\infty .
$$

Then there is an approximation error estimate of the form

$$
\left\|h-S_{I_{N}^{d}}^{\Lambda} h\right\|_{L_{\infty}\left(\mathbb{R}^{d}, \sqrt{\frac{\omega}{\varrho}}\right)} \lesssim N^{-m+\lambda}\|h\|_{\mathcal{H}^{m}\left(\mathbb{R}^{d}\right)} .
$$

Proof. Let $m \in \mathbb{N}, d \in \mathbb{N}$, and let $h \in L_{2}\left(\mathbb{R}^{d}, \omega\right) \cap H_{\text {mix }}^{m}\left(\mathbb{R}^{d}\right)$. By assumption, the criteria in Theorem 3.5 are fulfilled, and thus the transformed function $f$ of the form (3.27) is in $H_{\text {mix }}^{m}\left(\mathbb{T}^{d}\right)$. This $f$ is also in $\mathcal{H}^{m}\left(\mathbb{T}^{d}\right)$ due to the norm equivalence (2.2), and furthermore, it has a continuous representative because of the inclusion $\mathcal{H}^{m}\left(\mathbb{T}^{d}\right) \hookrightarrow \mathcal{A}^{m-\lambda}\left(\mathbb{T}^{d}\right) \hookrightarrow \mathcal{C}\left(\mathbb{T}^{d}\right)$ with $\lambda>\frac{1}{2}$ as in (2.3). Hence, for $f \in \mathcal{A}^{m-\lambda}\left(\mathbb{T}^{d}\right) \cap \mathcal{C}\left(\mathbb{T}^{d}\right)$ we have the approximation error bound

$$
\left\|f-S_{I_{N}^{d}}^{\Lambda} f\right\|_{L_{\infty}\left(\mathbb{T}^{d}\right)} \leq 2 N^{-m+\lambda}\|f\|_{\mathcal{A}^{m-\lambda}\left(\mathbb{T}^{d}\right)}
$$

as stated in Theorem 2.2.

With the inverse transformation $\mathbf{x}=\psi^{-1}(\mathbf{y})$ we have

$$
\hat{h}_{\mathbf{k}}=\left(h, \varphi_{\mathbf{k}}\right)_{L_{2}\left(\mathbb{R}^{d}, \omega\right)}=\left(f, \mathrm{e}^{2 \pi \mathrm{i} \mathbf{k} \cdot \circ}\right)_{L_{2}\left(\mathbb{T}^{d}\right)}=\hat{f}_{\mathbf{k}}
$$

and

$$
\|h\|_{\mathcal{H}^{m}\left(\mathbb{R}^{d}\right)}^{2}=\sum_{\mathbf{k} \in \mathbb{Z}^{d}} \omega_{\mathrm{hc}}(\mathbf{k})^{2 m}\left|\hat{h}_{\mathbf{k}}\right|^{2}=\sum_{\mathbf{k} \in \mathbb{Z}^{d}} \omega_{\mathrm{hc}}(\mathbf{k})^{2 m}\left|\hat{f}_{\mathbf{k}}\right|^{2}=\|f\|_{\mathcal{H}^{m}\left(\mathbb{T}^{d}\right)}^{2},
$$

as well as

$$
\begin{aligned}
& \left\|h-S_{I_{N}^{d}} h\right\|_{L_{\infty}\left(\mathbb{R}^{d}, \sqrt{\frac{\omega}{\varrho}}\right)}=\operatorname{ess} \sup _{\mathbf{y} \in \mathbb{R}^{d}}\left|\sqrt{\frac{\omega(\mathbf{y})}{\varrho(\mathbf{y})}}\left(h(\mathbf{y})-\sum_{\mathbf{k} \in I_{N}^{d}} \hat{h}_{\mathbf{k}} \varphi_{\mathbf{k}}(\mathbf{y})\right)\right| \\
& =\operatorname{ess} \sup _{\mathbf{y} \in \mathbb{R}^{d}}\left|h(\mathbf{y}) \sqrt{\frac{\omega(\mathbf{y})}{\varrho(\mathbf{y})}}-\sum_{\mathbf{k} \in I_{N}^{d}} \hat{h}_{\mathbf{k}} \mathrm{e}^{2 \pi \mathbf{i} \cdot \psi^{-1}(\mathbf{y})}\right| \\
& =\operatorname{ess}_{\sup _{\mathbf{x} \in \mathbb{T}^{d}}}\left|h(\psi(\mathbf{x})) \sqrt{\omega(\psi(\mathbf{x})) \psi^{\prime}(\mathbf{x})}-\sum_{\mathbf{k} \in I_{N}^{d}} \hat{h}_{\mathbf{k}} \mathrm{e}^{2 \pi \mathrm{i} \mathbf{k} \cdot \mathbf{x}}\right| \\
& =\left\|f-S_{I_{N}^{d}} f\right\|_{L_{\infty}\left(\mathbb{T}^{d}\right)}
\end{aligned}
$$

and

$$
\left\|h-S_{I_{N}^{d}}^{\Lambda} h\right\|_{L_{\infty}\left(\mathbb{R}^{d}, \sqrt{\frac{\omega}{e}}\right)}=\left\|f-S_{I_{N}^{d}}^{\Lambda} f\right\|_{L_{\infty}\left(\mathbb{T}^{d}\right)} .
$$

In total, by combining (3.43), (3.41), (2.4), and (3.42), we have shown that for a function $f \in \mathcal{H}^{m}\left(\mathbb{T}^{d}\right) \cap \mathcal{C}\left(\mathbb{T}^{d}\right)$, the approximation error can be bounded by

$$
\begin{aligned}
\left\|h-S_{I_{N}^{d}}^{\Lambda} h\right\|_{L_{\infty}\left(\mathbb{R}^{d}, \sqrt{\frac{w}{\varrho}}\right)} & =\left\|f-S_{I_{N}^{d}}^{\Lambda} f\right\|_{L_{\infty}\left(\mathbb{T}^{d}\right)} \leq 2 N^{-m+\lambda}\|f\|_{\mathcal{A}^{m-\lambda}\left(\mathbb{T}^{d}\right)} \\
& \leq 2 C_{d, \lambda} N^{-m+\lambda}\|f\|_{\mathcal{H}^{m}\left(\mathbb{T}^{d}\right)}=2 C_{d, \lambda} N^{-m+\lambda}\|h\|_{\mathcal{H}^{m}\left(\mathbb{R}^{d}\right)}<\infty
\end{aligned}
$$

with $\lambda>\frac{1}{2}$ and some constant $C_{d, \lambda}>1$. 
3.5.2. $\boldsymbol{L}_{\mathbf{2}}$-approximation error. Similarly, based on the $L_{2}\left(\mathbb{T}^{d}\right)$-approximation error bound (2.9) and the conditions proposed in Theorem 3.5, we prove an upper bound for the approximation error $\left\|h-S_{I_{N}^{d}}^{\Lambda} h\right\|$ in terms of a weighted $L_{2}$-norm on $\mathbb{R}^{d}$.

THEOREM 3.7. Let be given $d \in \mathbb{N}, m \in \mathbb{N}_{0}$, a hyperbolic cross $I_{N}^{d}$ with $N \geq 2^{d+1}$, and a reconstructing rank-1 lattice $\Lambda\left(\mathbf{z}, M, I_{N}^{d}\right)$. Let $\psi$ be a multivariate transformation as in (3.4) and $\omega$ be a multivariate weight function as in (3.10). We consider a multivariate function $h \in L_{2}\left(\mathbb{R}^{d}, \omega\right) \cap H_{\text {mix }}^{m}\left(\mathbb{R}^{d}\right)$. Furthermore, for all multi-indices $\mathbf{m}=\left(m_{1}, \ldots, m_{d}\right)^{\top} \in \mathbb{N}_{0}^{d}$ with $\|\mathbf{m}\|_{\ell_{\infty}} \leq m$ and all $j_{\ell}=0, \ldots, m, \ell=1, \ldots, d$, we assume

$$
\partial^{j_{\ell}}[\varrho]\left(y_{\ell}\right) \in \mathcal{C}_{0}(\mathbb{R}), \quad \partial^{j_{\ell}}[\psi]\left(x_{\ell}\right) \in \mathcal{C}((-1 / 2,1 / 2))
$$

and

$$
\max _{j_{\ell}=0, \ldots, m_{j}}\left\|\partial^{m_{\ell}-j_{\ell}}\left[\sqrt{\left(\omega_{\ell} \circ \psi_{\ell}\right) \psi_{\ell}^{\prime}}\right]\left(x_{\ell}\right) \psi_{\ell}^{\prime}\left(x_{\ell}\right)^{\max \left(-\frac{1}{2}, 2 j_{\ell}-\frac{3}{2}\right)}\right\|_{L_{\infty}(\mathbb{T})}<\infty .
$$

Then there is an approximation error estimate of the form

$$
\left\|h-S_{I_{N}^{d}}^{\Lambda} h\right\|_{L_{2}\left(\mathbb{R}^{d}, \omega\right)} \lesssim N^{-m}(\log N)^{(d-1) / 2}\|h\|_{\mathcal{H}^{m}\left(\mathbb{R}^{d}\right)} .
$$

Proof. Let $m \in \mathbb{N}, d \in \mathbb{N}$, and let $h \in L_{2}\left(\mathbb{R}^{d}, \omega\right) \cap H_{\text {mix }}^{m}\left(\mathbb{R}^{d}\right)$. By assumption, the criteria in Theorem 3.5 are fulfilled, and thus the transformed function $f$ of the form (3.27) is in $H^{m}\left(\mathbb{T}^{d}\right)$. This $f$ is also in $\mathcal{H}^{m}\left(\mathbb{T}^{d}\right)$ due to the norm equivalence (2.2), and it furthermore has a continuous representative because of the inclusion $\mathcal{H}^{m}\left(\mathbb{T}^{d}\right) \hookrightarrow \mathcal{C}\left(\mathbb{T}^{d}\right)$ in (2.3). For $f \in \mathcal{H}^{m}\left(\mathbb{T}^{d}\right) \cap \mathcal{C}\left(\mathbb{T}^{d}\right)$, Theorem 2.3 yields the approximation error bound of the form

$$
\left\|f-S_{I_{N}^{d}}^{\Lambda} f\right\|_{L_{2}\left(\mathbb{T}^{d}\right)} \leq C_{d, \beta} N^{-\beta}(\log N)^{(d-1) / 2}\|f\|_{\mathcal{H}^{\beta}\left(\mathbb{T}^{d}\right)}
$$

with some constant $C_{d, \beta}:=C(d, \beta)>0$. With the inverse transformation $\boldsymbol{x}=\psi^{-1}(\boldsymbol{y})$, we have

$$
\hat{h}_{\mathbf{k}}=\left(h, \varphi_{\mathbf{k}}\right)_{L_{2}\left(\mathbb{R}^{d}, \omega\right)}=\left(f, \mathrm{e}^{2 \pi \mathbf{i} \cdot \circ \cdot 0}\right)_{L_{2}\left(\mathbb{T}^{d}\right)}=\hat{f}_{\mathbf{k}},
$$

and

$$
\|h\|_{\mathcal{H}^{m}\left(\mathbb{R}^{d}\right)}^{2}=\sum_{\mathbf{k} \in \mathbb{Z}^{d}} \omega_{\mathrm{hc}}(\mathbf{k})^{2 m}\left|\hat{h}_{\mathbf{k}}\right|^{2}=\sum_{\mathbf{k} \in \mathbb{Z}^{d}} \omega_{\mathrm{hc}}(\mathbf{k})^{2 m}\left|\hat{f}_{\mathbf{k}}\right|^{2}=\|f\|_{\mathcal{H}^{m}\left(\mathbb{T}^{d}\right)}^{2}
$$

as in (3.42), as well as

$$
\left\|h-S_{I_{N}^{d}} h\right\|_{L_{2}\left(\mathbb{R}^{d}, \omega\right)}^{2}=\int_{\mathbb{R}^{d}}\left|h(\mathbf{y})-\sum_{\mathbf{k} \in I_{N}^{d}} \hat{h}_{\mathbf{k}} \varphi_{\mathbf{k}}(\mathbf{y})\right|^{2} \omega(\boldsymbol{y}) \mathrm{d} \mathbf{y}=\left\|f-S_{I_{N}^{d}} f\right\|_{L_{2}\left(\mathbb{T}^{d}\right)}^{2}
$$

and

$$
\left\|h-S_{I_{N}^{d}}^{\Lambda} h\right\|_{L_{2}\left(\mathbb{R}^{d}, \omega\right)}=\left\|f-S_{I_{N}^{d}}^{\Lambda} f\right\|_{L_{2}\left(\mathbb{T}^{d}\right)} .
$$

In total, by combining (3.45), (3.44), and (3.42), we have shown that for $f \in \mathcal{H}^{m}\left(\mathbb{T}^{d}\right) \cap \mathcal{C}\left(\mathbb{T}^{d}\right)$, the approximation error can be bounded by

$$
\begin{aligned}
\left\|h-S_{I_{N}^{d}}^{\Lambda} h\right\|_{L_{2}\left(\mathbb{R}^{d}, \omega\right)}=\left\|f-S_{I_{N}^{d}}^{\Lambda} f\right\|_{L_{2}\left(\mathbb{T}^{d}\right)} & \lesssim C_{d, \beta} N^{-\beta}(\log N)^{(d-1) / 2}\|f\|_{\mathcal{H}^{\beta}\left(\mathbb{T}^{d}\right)} \\
& =C_{d, \beta} N^{-\beta}(\log N)^{(d-1) / 2}\|h\|_{\mathcal{H}^{m}\left(\mathbb{R}^{d}\right)}<\infty
\end{aligned}
$$

with some constant $C_{d, \beta}>0$. 
4. Algorithms. In this chapter we start including the parameters $\boldsymbol{\eta}, \boldsymbol{\mu} \in \mathbb{R}^{d}$ in families of multivariate parameterized transformations $\psi(\circ, \boldsymbol{\eta})$ as in (3.4), in families of multivariate parameterized weight functions $\omega(\circ, \boldsymbol{\mu})$ as in (3.10), and in all related functions and objects.

We adapt the algorithms described in [8, Algorithm 3.1 and 3.2] that are based on one-dimensional fast Fourier transforms (FFTs). They are used for the fast reconstruction of approximated Fourier coefficients $\hat{h}_{\mathrm{k}}^{\Lambda}$ and the evaluation of a transformed multivariate trigonometric polynomials, in particular, in the approximated Fourier series $S_{I}^{\Lambda} h$ both given in (3.40). We introduce matrix-vector products of the form

$$
\mathbf{h}=\mathbf{A} \hat{\mathbf{h}} \quad \text { and } \quad \hat{\mathbf{h}}=M^{-1} \mathbf{A}^{*} \mathbf{h}
$$

with $\boldsymbol{\eta}, \boldsymbol{\mu} \in \mathbb{R}^{d}$,

$$
\mathbf{h}:=\left(h\left(\mathbf{y}_{j}\right) \sqrt{\frac{\omega\left(\mathbf{y}_{j}, \boldsymbol{\mu}\right)}{\varrho\left(\mathbf{y}_{j}, \boldsymbol{\eta}\right)}}\right)_{j=0, \ldots, M-1} \quad \text { for } \mathbf{y}_{j} \in \Lambda_{\psi(\circ, \boldsymbol{\eta})}(\mathbf{z}, M), \quad \hat{\mathbf{h}}:=\left(\hat{h}_{\mathbf{k}}\right)_{\mathbf{k} \in I_{N}},
$$

and the transformed Fourier matrices $\mathbf{A}$ and $\mathbf{A}^{*}$ given by

$$
\begin{aligned}
\mathbf{A} & :=\left(\mathrm{e}^{2 \pi \mathrm{i} \mathbf{k} \cdot \psi^{-1}\left(\mathbf{y}_{j}, \boldsymbol{\eta}\right)}\right)_{\mathbf{y}_{j} \in \Lambda_{\psi(0, \boldsymbol{\eta})}(\mathbf{z}, M), \mathbf{k} \in I} \in \mathbb{C}^{M \times|I|}, \\
\mathbf{A}^{*} & :=\left(\mathrm{e}^{-2 \pi \mathrm{i} \mathbf{k} \cdot \psi^{-1}\left(\mathbf{y}_{j}, \boldsymbol{\eta}\right)}\right)_{\mathbf{k} \in I, \mathbf{y}_{j} \in \Lambda_{\psi(0, \boldsymbol{\eta})}(\mathbf{z}, M)} \in \mathbb{C}^{|I| \times M} .
\end{aligned}
$$

We incorporate the previously described idea that the functions $h \in L_{2}\left(\mathbb{R}^{d}, \omega\right) \cap H_{\text {mix }}^{m}\left(\mathbb{R}^{d}\right)$ are transformed into functions $f$ on the torus $\mathbb{T}^{d}$ of the form (3.27) via transformations $\mathbf{x}_{j}=\psi\left(\mathbf{y}_{j}, \boldsymbol{\eta}\right)$ so that we have samples

$$
h\left(\mathbf{y}_{j}\right) \sqrt{\frac{\omega\left(\mathbf{y}_{j}, \boldsymbol{\mu}\right)}{\varrho\left(\mathbf{y}_{j}, \boldsymbol{\eta}\right)}}=h\left(\psi\left(\mathbf{x}_{j}, \boldsymbol{\eta}\right)\right) \sqrt{\omega\left(\psi\left(\mathbf{x}_{j}, \boldsymbol{\eta}\right), \boldsymbol{\mu}\right) \psi^{\prime}\left(\mathbf{x}_{j}, \boldsymbol{\eta}\right)}=f\left(\mathbf{x}_{j}, \boldsymbol{\eta}, \boldsymbol{\mu}\right)=f\left(\mathbf{x}_{j}\right),
$$

depending on the particular choices for $\boldsymbol{\eta}, \boldsymbol{\mu} \in \mathbb{R}^{d}$.

REMARK 4.1 . We identify $\mathbb{T}^{d}$ with different cubes. On the one hand, when defining rank-1 lattices $\Lambda(\mathbf{z}, M)$ in (2.5), we identify it with $[0,1)^{d}$. On the other hand, in order to apply the transformations $\psi$, we need to consider $\mathbb{T}^{d} \simeq\left[-\frac{1}{2}, \frac{1}{2}\right)^{d}$, which we achieve by reassigning all lattice points $\mathbf{x}_{j} \in \Lambda(\mathbf{z}, M)$ via

$$
\mathbf{x}_{j} \mapsto\left(\left(\mathbf{x}_{j}+\frac{\mathbf{1}}{2}\right) \bmod \mathbf{1}\right)-\frac{\mathbf{1}}{2},
$$

for all $j=0, \ldots, M-1$.

We already have showcased in Figure 3.1 that the definition of $\psi$ in (3.1) allows a range of functions with different slopes, which manifests in algebraic or exponential density functions $\varrho$. In Figure 4.1 we highlight these differences once more with transformed rank-1 lattices $\Lambda_{\psi(\circ, \boldsymbol{\eta})}(\mathbf{z}, M)$ as defined in (3.35). We consider the two-dimensional rank-1 lattice $\Lambda(\mathbf{z}, M)$ generated by $\mathbf{z}=(1,3)^{\top}$ and $M=31$. We compare the transformed lattices for the algebraic transformation and the error function transformation of the form (3.5) in their two-dimensional versions given by

$$
\psi(\mathbf{x}, \boldsymbol{\eta})=\left(\frac{2 \eta_{1} x_{1}}{\sqrt{1-4 x_{1}^{2}}}, \frac{2 \eta_{2} x_{2}}{\sqrt{1-4 x_{2}^{2}}}\right)^{\top}, \quad \psi(\mathbf{x}, \boldsymbol{\eta})=\left(\eta_{1} \operatorname{erf}^{-1}\left(2 x_{1}\right), \eta_{2} \operatorname{erf}^{-1}\left(2 x_{2}\right)\right)^{\top} .
$$



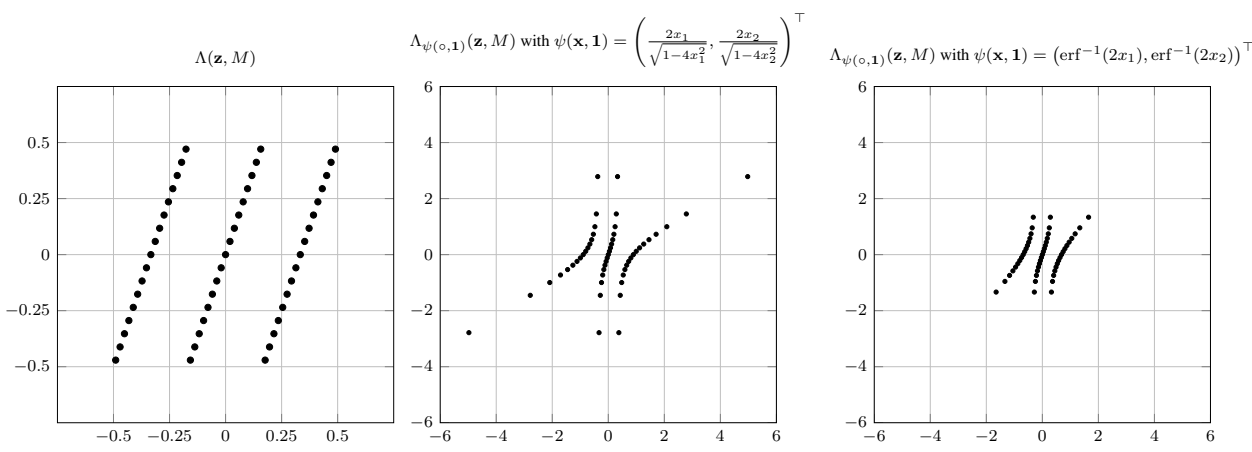

FIG. 4.1. A two-dimensional lattice $\Lambda(\mathbf{z}, M)$ with $\mathbf{z}=(1,3)^{\top}, M=31$ on the left and the resulting transformed lattice $\Lambda_{\psi(\circ, \boldsymbol{\eta})}(\mathbf{z}, M)$ for the algebraic transformation in the center and for the error function transformation on the right, as given in (4.1), and both used with $\boldsymbol{\eta}=\mathbf{1}$.

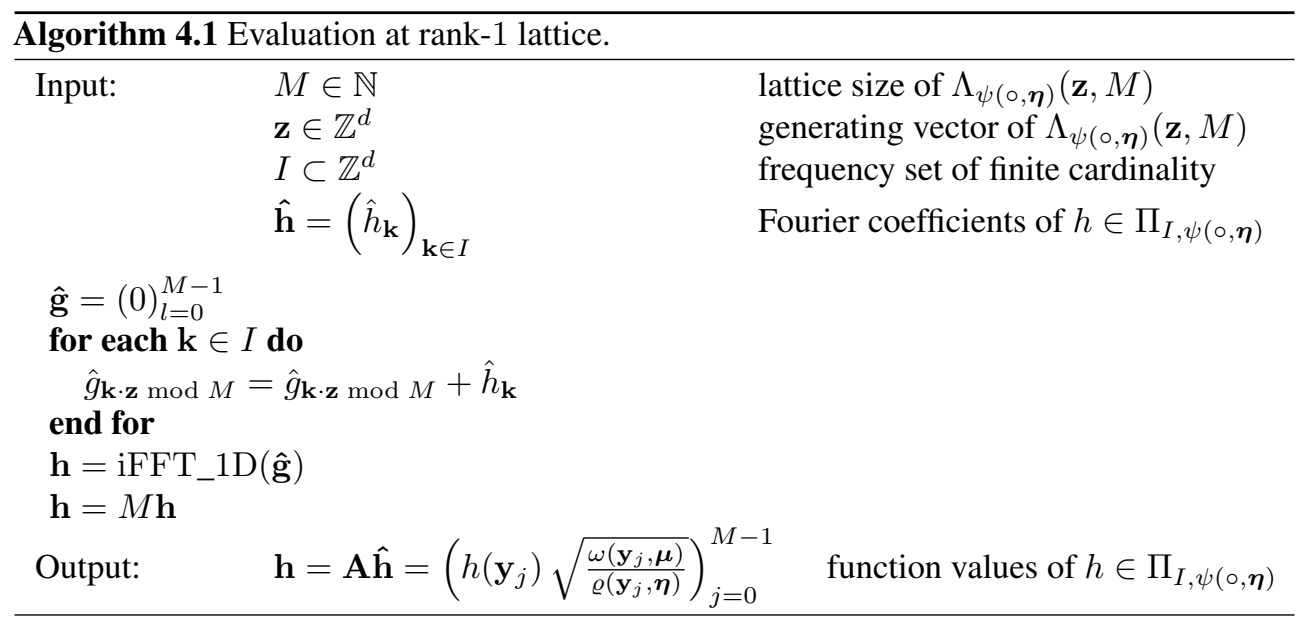

For $\boldsymbol{\eta}=\left(\eta_{1}, \eta_{2}\right)^{\top}=(1,1)^{\top}$, the graphs in the center and on the right-hand side of Figure 4.1 reveal that the algebraic transformation causes a wider spread of the lattice nodes close to the center, whereas the slope of the error function transformation increases drastically towards the boundary points, which we only notice for larger values $M$ and much finer lattices with more nodes closer to the boundary of the cube $\left(-\frac{1}{2}, \frac{1}{2}\right)^{2}$.

4.1. Evaluation of transformed multivariate trigonometric polynomials. Given a frequency set $I \subset \mathbb{Z}^{d}$ of finite cardinality $|I|<\infty$, we consider the multivariate trigonometric polynomial $h \in \Pi_{I, \psi(\circ, \boldsymbol{\eta})}$ as in (3.38) with Fourier coefficients $\hat{h}_{\mathbf{k}}$. The evaluation of $h$ at lattice points $\mathbf{y}_{j} \in \Lambda_{\psi(\circ, \boldsymbol{\eta})}(\mathbf{z}, M)$ simplifies to

$$
\begin{aligned}
h\left(\mathbf{y}_{j}\right) \sqrt{\frac{\omega\left(\mathbf{y}_{j}, \boldsymbol{\mu}\right)}{\varrho\left(\mathbf{y}_{j}, \boldsymbol{\eta}\right)}} & =\sum_{\mathbf{k} \in I} \hat{h}_{\mathbf{k}} \mathrm{e}^{2 \pi \mathrm{i} \mathbf{k} \cdot \psi^{-1}\left(\mathbf{y}_{j}, \boldsymbol{\eta}\right)} \\
& =\sum_{\ell=0}^{M-1}\left(\sum_{\substack{\mathbf{k} \in I, \mathbf{k} \cdot \mathbf{z} \equiv \ell(\bmod M)}} \hat{h}_{\mathbf{k}}\right) \mathrm{e}^{2 \pi \mathrm{i} \ell \frac{j}{M}}=\sum_{\ell=0}^{M-1} \hat{g}_{\ell} \mathrm{e}^{2 \pi \mathrm{i} \ell \frac{j}{M}}
\end{aligned}
$$




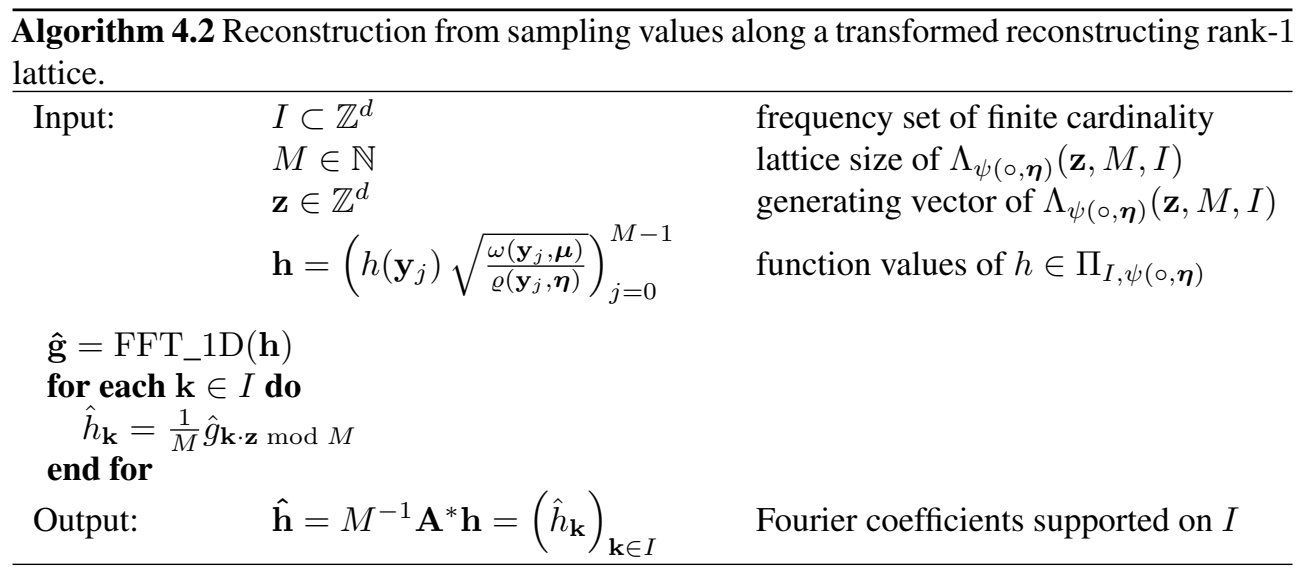

with

$$
\hat{g}_{\ell}=\sum_{\substack{\mathbf{k} \in I, \mathbf{k} \cdot \mathbf{z} \equiv \ell(\bmod M)}} \hat{h}_{\mathbf{k}}
$$

In total, the evaluation of such a function is realized by simply precomputing $\left(\hat{g}_{\ell}\right)_{\ell=0}^{M-1}$ and applying a one-dimensional inverse fast Fourier transform; see Algorithm 4.1.

4.2. Reconstruction of transformed multivariate trigonometric polynomials. For the reconstruction of a multivariate trigonometric polynomial $h \in \Pi_{I, \psi(\circ, \boldsymbol{\eta})}$ as in (3.38) from lattice points $\mathbf{y}_{j} \in \Lambda_{\psi(\circ, \boldsymbol{\eta})}(\mathbf{z}, M, I)$, we utilize the exact integration property (3.39) and the fact that we have

$$
\sum_{j=0}^{M-1}\left(\mathrm{e}^{2 \pi \mathrm{i} \frac{(\mathbf{k}-\mathbf{h}) \cdot \mathbf{z}}{M}}\right)^{j}= \begin{cases}M & \text { for } \mathbf{k} \cdot \mathbf{z} \equiv \mathbf{k} \cdot \mathbf{h}(\bmod M), \\ 0 & \text { otherwise }\end{cases}
$$

and thus, $\mathbf{A}^{*} \mathbf{A}=M \mathbf{I}$ with $\mathbf{I} \in \mathbb{C}^{|I| \times|I|}$ being the identity matrix. For fixed parameters $\boldsymbol{\eta}, \boldsymbol{\mu} \in \mathbb{R}^{d}$, we have input sample points of the form

$$
h\left(\mathbf{y}_{j}\right) \sqrt{\frac{\omega\left(\mathbf{y}_{j}, \boldsymbol{\mu}\right)}{\varrho\left(\mathbf{y}_{j}, \boldsymbol{\eta}\right)}}=h\left(\psi\left(\mathbf{x}_{j}, \boldsymbol{\eta}\right)\right) \sqrt{\omega\left(\psi\left(\mathbf{x}_{j}, \boldsymbol{\eta}\right), \boldsymbol{\mu}\right) \psi^{\prime}\left(\mathbf{x}_{j}, \boldsymbol{\eta}\right)}=f\left(\mathbf{x}_{j}, \boldsymbol{\eta}, \boldsymbol{\mu}\right)=f\left(\mathbf{x}_{j}\right) .
$$

For the reconstruction of the Fourier coefficients $\hat{h}_{\mathbf{k}}$ we use a single one-dimensional fast Fourier transform. The entries of the resulting vector $\left(\hat{g}_{\ell}\right)_{\ell=0}^{M-1}$ are renumbered by means of the unique inverse mapping $\mathbf{k} \mapsto \mathbf{k} \cdot \mathbf{z} \bmod M$; see Algorithm 4.2.

4.3. Discrete approximation error. In order to use Algorithms 4.1 and 4.2 to illustrate the proposed error bounds of Theorems 3.6 and 3.7, we sample the approximated Fourier partial sum $S_{I}^{\Lambda} h$ in order to discretize and thus approximate the error

$$
\left\|h-S_{I}^{\Lambda} h\right\|_{L_{\infty}\left(\mathbb{R}^{d}, \sqrt{\frac{\omega(o, \mu)}{\varrho(0, \eta)}}\right)},
$$

which is equal to $\left\|f-S_{I}^{\Lambda} f\right\|_{L_{\infty}\left(\mathbb{T}^{d}\right)}$ as shown in the proof of Theorem 3.6. Based on the given sample data in the vector

$$
\mathbf{h}=\left(h\left(\mathbf{y}_{j}\right) \sqrt{\frac{\omega\left(\mathbf{y}_{j}, \boldsymbol{\mu}\right)}{\varrho\left(\mathbf{y}_{j}, \boldsymbol{\eta}\right)}}\right)_{j=0}^{M-1}
$$


with lattice points $\mathbf{y}_{j} \in \Lambda_{\psi(\circ, \boldsymbol{\eta})}(\mathbf{z}, M, I)$, we apply Algorithm 4.2 yielding a vector of approximated Fourier coefficients via $\hat{\mathbf{h}}=M^{-1} \mathbf{A}^{*} \mathbf{h}$, which we use as input for Algorithm 4.1. After applying both algorithms we have computed the vector

$$
\mathbf{h}_{\text {approx }}:=M^{-1} \mathbf{A A}^{*} \mathbf{h}=\left(\sqrt{\frac{\omega\left(\mathbf{y}_{j}, \boldsymbol{\mu}\right)}{\varrho\left(\mathbf{y}_{j}, \boldsymbol{\eta}\right)}} S_{I_{N}^{d}}^{\Lambda} h\left(\mathbf{y}_{j}\right)\right)_{j=0}^{M-1} .
$$

In [10, Corollary 1] it was shown that under mild assumptions, for each frequency set $I \subset \mathbb{Z}^{d}$ that induces a reconstructing rank-1 lattice, there is an $M \in \mathbb{N}$ such that $|I| \leq M \lesssim|I|^{2}$. Furthermore, in (4.2) we already observed that for a reconstruction rank-1 lattice $\Lambda_{\psi(\circ, \boldsymbol{\eta})}(\mathbf{z}, M, I)$, we have $\mathbf{A}^{*} \mathbf{A}=M \mathbf{I}$ with $\mathbf{I} \in \mathbb{C}^{|I| \times|I|}$ being the identity matrix. However, $\mathbf{A} \mathbf{A}^{*} \in \mathbb{C}^{M \times M}$ is generally not an identity matrix. Hence, there is a gap between the initially given values $\mathbf{h}$ and the resulting vector $\mathbf{h}_{\text {approx }}$ which we quantify with the discrete approximation error

$$
\left\|\mathbf{h}-\mathbf{h}_{\text {approx }}\right\|_{\ell_{\infty}}:=\max _{j=0, \ldots, M-1}\left|\sqrt{\frac{\omega\left(\mathbf{y}_{j}, \boldsymbol{\mu}\right)}{\varrho\left(\mathbf{y}_{j}, \boldsymbol{\eta}\right)}}\left(h\left(\mathbf{y}_{j}\right)-S_{I_{N}^{d}}^{\Lambda} h\left(\mathbf{y}_{j}\right)\right)\right| .
$$

But it is important to note that we only discuss this particular discretization approach, which is exclusively sampling on the rank-1 lattice nodes and does not measure the quality of the approximation at any point outside the rank-1 lattice. Nevertheless, for hyperbolic crosses $I_{N}^{d}$ we still have the upper bound

$$
\begin{aligned}
\left\|\mathbf{h}-\mathbf{h}_{\text {approx }}\right\|_{\ell_{\infty}} & \leq\left\|h-S_{I_{N}^{d}}^{\Lambda} h\right\|_{L_{\infty}\left(\mathbb{R}^{d}, \sqrt{\frac{\omega(0, \mu)}{\varrho(0, \eta)}}\right)} \\
& =\left\|f-S_{I_{N}^{d}}^{\Lambda} f\right\|_{L_{\infty}\left(\mathbb{T}^{d}\right)} \leq 2 N^{-m}\|h\|_{\mathcal{H}^{m}\left(\mathbb{R}^{d}\right)}
\end{aligned}
$$

for appropriately chosen parameters $\boldsymbol{\eta}, \boldsymbol{\mu} \in \mathbb{R}^{d}$ as shown in Theorem 3.6. Hence, the theoretical results predict a certain decay rate of the discretized approximation error for increasing $N \in \mathbb{N}$ with fixed $m \in \mathbb{N}$ and suitably chosen parameter $\boldsymbol{\eta}$ and $\boldsymbol{\mu}$.

On the other hand, for the $L_{2}$-approximation error we lack a similar discretization approach. However, by Theorem 3.7 we know that for fixed $m \in \mathbb{N}$ and suitably chosen parameters $\boldsymbol{\eta}$ and $\boldsymbol{\mu}$, the error $\left\|h-S_{I_{N}^{d}}^{\Lambda} h\right\|_{L_{2}\left(\mathbb{R}^{d}, \omega\right)}=\left\|f-S_{I_{N}^{d}}^{\Lambda} f\right\|_{L_{2}\left(\mathbb{T}^{d}\right)}$ is bounded above by $N^{-m}(\log N)^{(d-1) / 2}\|f\|_{\mathcal{H}^{m}\left(\mathbb{T}^{d}\right)}$. By Parseval's equation we have

$$
\begin{aligned}
\left\|f-S_{I_{N}^{d}}^{\Lambda} f\right\|_{L_{2}\left(\mathbb{T}^{d}\right)}^{2}=\sum_{\mathbf{k} \in \mathbb{Z}^{d}}\left|\hat{f}_{\mathbf{k}}-\hat{f}_{\mathbf{k}}^{\Lambda}\right|^{2} & =\sum_{\mathbf{k} \in \mathbb{Z}^{d} \backslash I_{N}^{d}}\left|\hat{f_{\mathbf{k}}}\right|^{2}+\sum_{\mathbf{k} \in I_{N}^{d}}\left|\hat{f_{\mathbf{k}}}-\hat{f}_{\mathbf{k}}^{\Lambda}\right|^{2} \\
& =\|f\|_{L_{2}\left(\mathbb{T}^{d}\right)}^{2}+\sum_{\mathbf{k} \in I_{N}^{d}}\left(\left|\hat{f_{\mathbf{k}}}-\hat{f}_{\mathbf{k}}^{\Lambda}\right|^{2}-\left|\hat{f}_{\mathbf{k}}\right|^{2}\right) .
\end{aligned}
$$

Hence, we can evaluate the $L_{2}$-approximation error if we use Algorithm 4.2 to reconstruct the approximated Fourier coefficients $\hat{f}_{\mathbf{k}}^{\Lambda}$ and if it is possible to calculate the Fourier coefficients $\hat{f}_{\mathbf{k}}$ for all $\mathbf{k} \in I_{N}^{d}$. Later on, we present an example where the Fourier coefficients $\hat{f}_{\mathbf{k}}$ can be computed for all $\mathbf{k} \in \mathbb{Z}^{d}$. Generally this is not possible, so that we have to resort on the theoretical approach based on norm equivalences presented earlier in this paper in order to obtain the information if the Fourier coefficients $\hat{f}_{\mathbf{k}}$ are square summable. 
5. Examples. Based on the algebraic transformation (3.6) and the error function transformations (3.8) we discuss certain choices of test functions $h$ and weight functions $\omega$ for which the proposed smoothness conditions (3.28) in Theorem 3.5 are fulfilled. In both cases we proceed similarly: We fix a family of multivariate weight functions $\omega(\circ, \boldsymbol{\mu}), \boldsymbol{\mu} \in \mathbb{R}^{d}$, as well as the test function $h$ in $L_{2}\left(\mathbb{R}^{d}, \omega(\circ, \boldsymbol{\mu})\right) \cap H_{\text {mix }}^{m}\left(\mathbb{R}^{d}\right)$. Then we fix a family of multivariate transformations $\psi(\circ, \boldsymbol{\eta}), \boldsymbol{\eta} \in \mathbb{R}^{d}$, of the form (3.5). Afterwards we calculate lower bounds for $\boldsymbol{\mu}$ and $\boldsymbol{\eta}$ such that $f(\mathbf{x}, \boldsymbol{\eta}, \boldsymbol{\mu}):=h(\psi(\mathbf{x}, \boldsymbol{\eta})) \sqrt{\omega(\psi(\mathbf{x}, \boldsymbol{\eta}), \boldsymbol{\mu}) \psi^{\prime}(\mathbf{x}, \boldsymbol{\eta})}$ is in $H_{\mathrm{mix}}^{m}\left(\mathbb{T}^{d}\right)$ for Sobolev smoothness orders $m=0,1,2,3$. Finally, we switch to dimension $d=2$, and, based on the calculated parameter bounds, we use Algorithms 4.1 and 4.2 for numerical tests of the $L_{\infty}$-approximation error bound proposed in Theorems 3.6 and discuss the possibility to evaluate the Fourier coefficients $\hat{h}_{\mathbf{k}}$.

Throughout this section we repeatedly specify parameter vectors that have the same number in each entry, for which we recall the short notation of just using a single bold number, e.g., $\mathbf{1}=(1, \ldots, 1)^{\top}$ that appeared earlier in the definition of rank-1 lattices $\Lambda(\mathbf{z}, M)$ in (2.5).

5.1. Algebraic transformation. The test function is of the form

$$
h(\mathbf{y})=\frac{1}{1+\|\mathbf{y}\|_{\ell_{2}}^{2}}
$$

with $\|\mathbf{y}\|_{\ell_{2}}:=\sqrt{y_{1}^{2}+\ldots+y_{d}^{2}}$ for $\mathbf{y} \in \mathbb{R}^{d}$. According to [1, pp. 363-364], for $d=1$ this function is rather difficult to approximate by classical approximation methods. We fix the algebraic weight function (3.15) in its multivariate version of the form

$$
\omega(\mathbf{y}, \boldsymbol{\mu}):=\prod_{j=1}^{d}\left(\frac{1}{1+y_{j}^{2}}\right)^{\mu_{j}}
$$

with $\boldsymbol{\mu}=\left(\mu_{1}, \ldots, \mu_{d}\right)^{\top} \in \mathbb{R}^{d}$ and the algebraic transformation $\psi(\mathbf{x}, \boldsymbol{\eta})=\left(\left(\psi_{j}\left(x_{j}, \eta_{j}\right)\right)_{j=1}^{d}\right)^{\top}$ in the form (3.5) with $\mathbf{x} \in\left(-\frac{1}{2}, \frac{1}{2}\right)^{d}$ and the parameter $\boldsymbol{\eta}=\left(\eta_{1}, \ldots, \eta_{d}\right)^{\top} \in \mathbb{R}^{d}$. The univariate components of the transformation are given by

$$
\begin{aligned}
\psi_{j}\left(x_{j}, \eta_{j}\right) & =\frac{2 \eta_{j} x_{j}}{\left(1-4 x_{j}^{2}\right)^{\frac{1}{2}}}, & \psi_{j}^{\prime}\left(x_{j}, \eta_{j}\right) & =\frac{2 \eta_{j}}{\left(1-4 x_{j}^{2}\right)^{\frac{3}{2}}} \\
\psi_{j}^{-1}\left(y_{j}, \eta_{j}\right) & =\frac{y_{j}}{2\left(\eta_{j}^{2}+y_{j}^{2}\right)^{\frac{1}{2}}}, & \varrho_{j}\left(y_{j}, \eta_{j}\right) & =\frac{1}{2\left(\eta_{j}^{2}+y_{j}^{2}\right)^{\frac{3}{2}}}
\end{aligned}
$$

For $\eta_{j}=1$ we have stated the definition of $\psi_{j}(\circ, 1)$ earlier in (3.6). For the resulting weighted Hilbert space $L_{2}\left(\mathbb{R}^{d}, \omega(\circ, \boldsymbol{\mu})\right)$, we have a system $\left\{\varphi_{\mathbf{k}}\right\}_{\mathbf{k} \in \mathbb{Z}^{d}}$ of product functions given in (3.36) with univariate components $\left(\varphi_{k_{j}}\right)_{j=1}^{d}$ as in (3.11) of the form

$$
\varphi_{k_{j}}\left(y_{j}, \eta_{j}, \mu_{j}\right):=\frac{1}{\sqrt{2}}\left(1+y_{j}^{2}\right)^{\frac{\mu_{j}}{2}}\left(\eta_{j}^{2}+y_{j}^{2}\right)^{-\frac{3}{4}} \mathrm{e}^{\pi \mathrm{i} k_{j} y_{j}\left(\eta_{j}^{2}+y_{j}^{2}\right)^{-\frac{1}{2}}}
$$

which are orthogonal with respect to the weighted scalar product

$$
\left(h_{1}, h_{2}\right)_{L_{2}\left(\mathbb{R}^{d}, \omega(\circ, \boldsymbol{\mu})\right)}=\pi^{-\frac{d}{2}} \int_{\mathbb{R}^{d}} \prod_{j=1}^{d}\left(1+y_{j}^{2}\right)^{-\mu_{j}} h_{1}(\mathbf{y}) \overline{h_{2}(\mathbf{y})} \mathrm{d} \mathbf{y} .
$$


The Fourier coefficients $\hat{h}_{\mathbf{k}}$ of an arbitrary function $h \in L_{2}\left(\mathbb{R}^{d}, \omega(\circ, \boldsymbol{\mu})\right)$ are of the form

$$
\begin{aligned}
\hat{h}_{\mathbf{k}} & :=\left(h, \varphi_{\mathbf{k}}\right)_{L_{2}\left(\mathbb{R}^{d}, \omega(\circ, \boldsymbol{\mu})\right)} \\
& =\int_{\mathbb{R}^{d}} h(\mathbf{y}) \overline{\varphi_{\mathbf{k}}(\mathbf{y}, \boldsymbol{\eta}, \boldsymbol{\mu})} \omega(\mathbf{y}, \boldsymbol{\mu}) \mathrm{d} \mathbf{y} \\
& =2^{-\frac{d}{2}} \int_{\mathbb{R}^{d}} h(\mathbf{y}) \prod_{j=1}^{d}\left(1+y_{j}^{2}\right)^{-\frac{\mu_{j}}{2}}\left(\eta_{j}^{2}+y_{j}^{2}\right)^{-\frac{3}{4}} \mathrm{e}^{-\pi \mathrm{i} k_{j} y_{j}\left(\eta_{j}^{2}+y_{j}^{2}\right)^{-\frac{1}{2}}} \mathrm{~d} \mathbf{y} .
\end{aligned}
$$

The test function $h$ in (5.1) combined with the weight function (5.2) and the transformations (5.3) lead to transformed functions $f$ in the sense of (3.27) of the form

$$
\begin{aligned}
f(\mathbf{x}, \boldsymbol{\eta}, \boldsymbol{\mu}) & =h\left(\psi_{1}\left(x_{1}, \eta_{1}\right), \ldots, \psi_{d}\left(x_{d}, \eta_{d}\right)\right) \prod_{j=1}^{d} \sqrt{\omega_{j}\left(\psi_{j}\left(x_{j}, \eta_{j}\right), \mu_{j}\right) \psi_{j}^{\prime}\left(x_{j}, \eta_{j}\right)} \\
& =\left(1+\sum_{j=1}^{d} \frac{4 \eta_{j}^{2} x_{j}^{2}}{1-4 x_{j}^{2}}\right)^{-1} \prod_{j=1}^{d} \sqrt{\left(\frac{1-4 x_{j}^{2}}{1-4\left(1-\eta_{j}^{2}\right) x_{j}^{2}}\right)^{\mu_{j}} 2 \eta_{j}\left(1-4 x_{j}^{2}\right)^{-\frac{3}{2}}} .
\end{aligned}
$$

In Figure 5.1 we provide a side-by-side comparison of the graphs of these transformed functions $d=2$ for fixed $\boldsymbol{\mu}=(4,4)^{\top}$ with varying $\boldsymbol{\eta}=\left(\eta_{1}, \eta_{2}\right) \in \mathbb{R}^{2}, 1 / 2 \leq \eta_{1}, \eta_{2} \leq 2$, and for fixed $\boldsymbol{\eta}=(1,1)^{\top}$ with varying $\boldsymbol{\mu}=\left(\mu_{1}, \mu_{2}\right)^{\top}, 0 \leq \mu_{1}, \mu_{2} \leq 10$.

We proceed to determine the values $\boldsymbol{\eta}, \boldsymbol{\mu} \in \mathbb{R}$ for which $f(\circ, \boldsymbol{\eta}, \boldsymbol{\mu})$ in (5.4) is an element of $H_{\text {mix }}^{m}\left(\mathbb{T}^{d}\right)$ by investigating the conditions (3.28) in Theorem 3.5. First of all, we observe that for $\eta_{1}, \ldots, \eta_{d}>0$, the components $\psi_{1}, \ldots, \psi_{d}$ of the function $\psi(\circ, \boldsymbol{\eta})$ in (5.3) are transformations in the sense of (3.1) by being increasing, continuously differentiable, and invertible functions. Furthermore, for all $\ell=1, \ldots, d$, it is easy to verify that the first three derivatives of all $\psi_{j}\left(\circ, \eta_{j}\right)$ are in fact continuous on $\left(-\frac{1}{2}, \frac{1}{2}\right)$ for $\eta_{j}>0$ and that the first three derivatives of $\varrho_{j}\left(\circ, \eta_{j}\right)$ are in $\mathcal{C}_{0}(\mathbb{R})$ for all non-zero $\eta_{j} \in \mathbb{R}$. Finally, we verify the $L_{\infty}$-conditions (3.28) in Theorem 3.5 for $m=0,1,2,3$. We suppose that for $\ell=1, \ldots, d$ we have $m=m_{\ell}$, and we need to inspect if the appearing $L_{\infty}(\mathbb{T})$-norms are finite for all $j_{\ell}=0, \ldots, m$ :

- Let $m=0$. Then we only have a condition for the norm

$$
\begin{aligned}
& \left\|\sqrt{\omega_{\ell}\left(\psi_{\ell}\left(x_{\ell}, \eta_{\ell}\right), \mu_{\ell}\right) \psi_{\ell}^{\prime}\left(x_{\ell}, \eta_{\ell}\right)}\left(\psi_{\ell}^{\prime}\left(x_{\ell}, \eta_{\ell}\right)\right)^{-\frac{1}{2}}\right\|_{L_{\infty}(\mathbb{T})} \\
& =\left\|\left(\frac{1-4 x_{\ell}^{2}}{1-4\left(1-\eta_{\ell}^{2}\right) x_{\ell}^{2}}\right)^{\mu_{\ell}}\right\|_{L_{\infty}(\mathbb{T})},
\end{aligned}
$$

which is finite for $\mu_{\ell} \geq 0$.

- Let $m=1$. We have to consider two conditions. For $j_{\ell}=0$ we have

$$
\begin{aligned}
& \left\|\frac{\partial}{\partial x_{\ell}}\left[\sqrt{\omega_{\ell}\left(\psi_{\ell}\left(x_{\ell}, \eta_{\ell}\right), \mu_{\ell}\right) \psi_{\ell}^{\prime}\left(x_{\ell}, \eta_{\ell}\right)}\right] \psi_{\ell}^{\prime}\left(x_{\ell}, \eta_{\ell}\right)^{-\frac{1}{2}}\right\|_{L_{\infty}(\mathbb{T})} \\
& =\left\|x_{\ell}\left(\frac{\left(1-4 x_{\ell}^{2}\right)}{1+4\left(\eta_{\ell}^{2}-1\right) x_{\ell}^{2}}\right)^{\frac{\mu_{\ell}}{2}-1} \frac{\left(-\left(\mu_{\ell}^{2}+3\right) \mu_{\ell}+6\left(1+\left(\eta_{\ell}^{2}-1\right) x_{\ell}^{2}\right)\right)}{\left(1+4\left(\eta_{\ell}^{2}-1\right) x_{\ell}^{2}\right)^{2}}\right\|_{L_{\infty}(\mathbb{T})},
\end{aligned}
$$

and this is finite if $\mu_{\ell}>2$. 


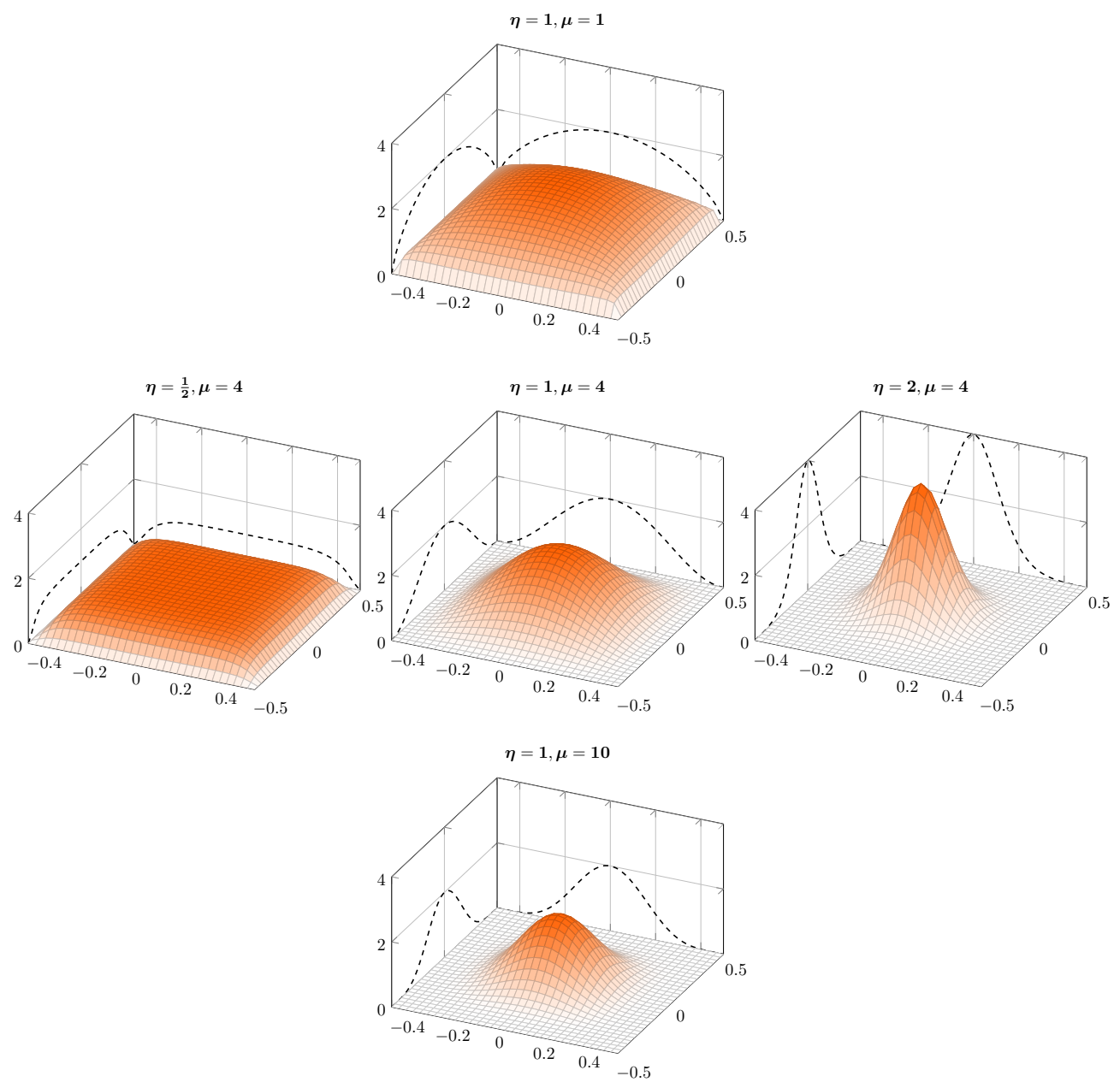

FIG. 5.1. Plots of the two-dimensional transformed function $f(\circ, \boldsymbol{\eta}, \boldsymbol{\mu})$ for various combinations of the parameters $\boldsymbol{\mu}$ and $\boldsymbol{\eta}$ with an algebraic weight function $\omega(\circ, \boldsymbol{\mu})$ in (5.2) and the algebraic transformation $\psi(\circ, \boldsymbol{\eta})$ in (5.3). Horizontally, $\boldsymbol{\mu}=(4,4)^{\top}$ is fixed, vertically $\boldsymbol{\eta}=(1,1)^{\top}$ is fixed. The individual univariate functions $f\left(\left(x_{1}, 0\right), \boldsymbol{\eta}, \boldsymbol{\mu}\right), f\left(\left(0, x_{2}\right), \boldsymbol{\eta}, \boldsymbol{\mu}\right)$ are shown with dashed lines.

For $j_{\ell}=1$ we have

$$
\begin{aligned}
& \left\|\sqrt{\omega_{\ell}\left(\psi_{\ell}\left(x_{\ell}, \eta_{\ell}\right), \mu_{\ell}\right) \psi_{\ell}^{\prime}\left(x_{\ell}, \eta_{\ell}\right)}\left(\psi_{\ell}^{\prime}\left(x_{\ell}, \eta_{\ell}\right)\right)^{\frac{1}{2}}\right\|_{L_{\infty}(\mathbb{T})} \\
& =\left\|2 \eta_{\ell}\left(\frac{1-4 x_{\ell}^{2}}{1+4\left(\eta_{\ell}^{2}-1\right) x_{\ell}^{2}}\right)^{\frac{\mu_{\ell}}{2}}\left(1-4 x_{\ell}^{2}\right)^{-\frac{3}{2}}\right\|_{L_{\infty}(\mathbb{T})},
\end{aligned}
$$

and this is finite for $\mu_{\ell}>3$.

- Likewise, after verifying the individual conditions, we conclude that for $m=2$ we have a lower bound of $\mu_{\ell}>9$ and for $m=3$ of $\mu_{\ell}>15$.

In total, $f$ is at least an $L_{2}\left(\mathbb{T}^{d}\right)$-function for all $\mu_{1}, \ldots, \mu_{d} \geq 0$, it is at least in $H_{\text {mix }}^{1}\left(\mathbb{T}^{d}\right)$ for $\mu_{1}, \ldots, \mu_{d}>3$, at least in $H_{\text {mix }}^{2}\left(\mathbb{T}^{d}\right)$ for $\mu_{1}, \ldots, \mu_{d}>9$, and at least an $H_{\text {mix }}^{2}\left(\mathbb{T}^{d}\right)$-function for $\mu_{1}, \ldots, \mu_{d}>15$. Apparently, the parameters $\eta_{1}, \ldots, \eta_{d}$ in the transformation $\psi(\circ, \boldsymbol{\eta})$ do 


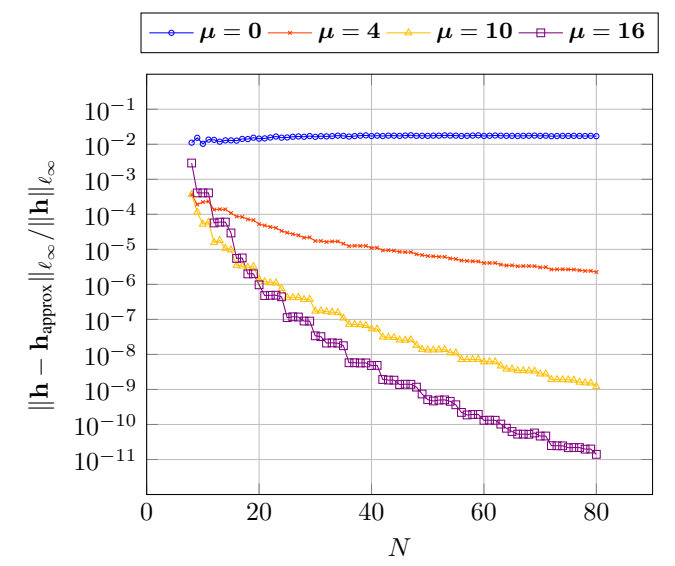

FIG. 5.2. Comparison of the discrete $\ell_{\infty}$-approximation error $\left\|\mathbf{h}-\mathbf{h}_{\text {approx }}\right\|_{\ell_{\infty}} /\|\mathbf{h}\|_{\ell_{\infty}}$ of the two-dimensional test function (5.1) in combination with the algebraic transformation $\psi(\circ, \boldsymbol{\eta})(5.3)$ and the algebraic weight function $\omega(\circ, \boldsymbol{\mu})(5.2)$ in their two-dimensional versions with fixed $\boldsymbol{\eta}=\mathbf{1}$ and $\boldsymbol{\mu} \in\{\mathbf{0}, \mathbf{4}, \mathbf{1 0}, \mathbf{1 6}\}$.

not have an impact on the Sobolev smoothness of $f(\circ, \boldsymbol{\eta}, \boldsymbol{\mu})$ as in (5.4) according to this specific set of conditions. In other words, if $\boldsymbol{\eta}$ is able to control the smoothness of $f$, then we can not recognize it with these conditions-at least for this particular combination of the transformation $\psi$ and the weight function $\omega$.

5.1.1. Discussion of the $\boldsymbol{L}_{\infty}$-approximation error. Next we discuss the application of the multivariate $L_{\infty}\left(\mathbb{R}^{d}\right)$-approximation error bound in Theorem 3.6 for $d=2$ with the two-dimensional test function $h$ in (5.1), the weight function (5.2), the transformations (5.3), and the resulting transformed functions $f$ given in (5.4).

Let a reconstructing rank-1 lattice $\Lambda\left(\mathbf{z}, M, I_{N}^{2}\right)$ with $N \geq 8$ be given. We have already evaluated the sufficient conditions proposed in Theorem 3.5, yielding lower bounds for $\boldsymbol{\mu} \geq \mathbf{0}$ such that $f$ is at least of Sobolev smoothness order $m=0,1,2,3$, i.e., $f \in H_{\text {mix }}^{m}\left(\mathbb{T}^{2}\right)$, and thus $f \in \mathcal{H}^{m}\left(\mathbb{T}^{2}\right)$. We fix $\lambda=1$, and for $m \in \mathbb{N}_{0}$, we choose $\boldsymbol{\mu}, \boldsymbol{\eta} \in \mathbb{R}^{2}$ such that $f \in \mathcal{H}^{m+1}\left(\mathbb{T}^{2}\right) \hookrightarrow \mathcal{A}^{m}\left(\mathbb{T}^{2}\right)$. As outlined in (4.4) we expect the discrete approximation error (4.3) to be bounded by

$$
\left\|\mathbf{h}-\mathbf{h}_{\text {approx }}\right\|_{\ell_{\infty}} \leq\left\|f-S_{I_{N}^{2}}^{\Lambda} f\right\|_{L_{\infty}\left(\mathbb{T}^{2}\right)} \lesssim \begin{cases}N^{0} & \text { for } \mu_{j} \geq 0 \\ N^{-1} & \text { for } \mu_{j}>3 \\ N^{-2} & \text { for } \mu_{j}>9 \\ N^{-3} & \text { for } \mu_{j}>15\end{cases}
$$

For $N=8, \ldots, 80, \boldsymbol{\eta}=\mathbf{1}$, and $\boldsymbol{\mu} \in\{\mathbf{0}, \mathbf{4}, \mathbf{1 0}, \mathbf{1 6}\}$, we actually observe this behavior for the relative discrete approximation error $\left\|\mathbf{h}-\mathbf{h}_{\text {approx }}\right\|_{\ell_{\infty}} /\|\mathbf{h}\|_{\ell_{\infty}}$ as seen in Figure 5.2.

5.1.2. Discussion of the $\boldsymbol{L}_{\mathbf{2}}$-approximation error. We switch to dimension $d=1$. In Theorem 3.7 we proved that when $f$ of the form (3.17) is in $\mathcal{H}^{m}(\mathbb{T}) \cap \mathcal{C}(\mathbb{T})$, we have

$$
\left\|h-S_{I_{N}^{1}}^{\Lambda} h\right\|_{L_{2}(\mathbb{R}, \omega)}=\left\|f-S_{I_{N}^{1}}^{\Lambda} f\right\|_{L_{2}(\mathbb{T})} \lesssim N^{-m} .
$$

For one particular special case with explicitly computable Fourier coefficients $\hat{f}_{k}$, we observe that their rate of decay is consistent with the theoretical propositions. The conditions of 
Theorem 3.4 yield, for $m=1,2,3$, that

$$
f \in \begin{cases}H^{1}(\mathbb{T}) & \text { for } \mu>3 \\ H^{2}(\mathbb{T}) & \text { for } \mu>9 \\ H^{3}(\mathbb{T}) & \text { for } \mu>15\end{cases}
$$

We compare these lower bounds with the specific lower bounds for the chosen $h$ in (5.1). Fixing $\eta=1$, the transformed function $f$ in (5.4) simplifies to

$$
f(x, 1, \mu)=f(x, \mu):=\left(1-4 x^{2}\right)^{\frac{1}{2}\left(\mu+\frac{1}{2}\right)} .
$$

We then explicitly calculate that

$$
\int_{\mathbb{T}}\left|\frac{\mathrm{d}}{\mathrm{d} x}[f](x, \mu)\right|^{2} \mathrm{~d} x=\left(\mu+\frac{1}{2}\right)^{2} \int_{\mathbb{T}} 16 x^{2}\left|1-4 x^{2}\right|^{\mu-\frac{3}{2}} \mathrm{~d} x<\infty
$$

for $\mu \geq \frac{3}{2}$, as well as

$$
\int_{\mathbb{T}}\left|\frac{\mathrm{d}^{2}}{\mathrm{~d} x^{2}}[f](x, \mu)\right|^{2} \mathrm{~d} x=4(2 \mu+1)^{2} \int_{\mathbb{T}}\left(1-(4 \mu-2) x^{2}\right)^{2}\left|1-4 x^{2}\right|^{\mu-\frac{7}{2}} \mathrm{~d} x<\infty
$$

for $\mu \geq \frac{7}{2}$, and so forth, which is summarized for $m=1,2,3$ as

$$
f \in \begin{cases}H^{1}(\mathbb{T}) & \text { for } \mu \geq \frac{3}{2}, \\ H^{2}(\mathbb{T}) & \text { for } \mu \geq \frac{7}{2}, \\ H^{3}(\mathbb{T}) & \text { for } \mu \geq \frac{11}{2} .\end{cases}
$$

Due to the norm equivalence (2.2), we know that the absolute Fourier coefficients $\left|\hat{f}_{k}\right|$ of a function $f \in H_{\text {mix }}^{m}(\mathbb{T})$ decay at least as fast as $|k|^{-m}$. In our particular example with the above function $f(x, \mu)$ we have a decay twice as fast, which is observed by considering $k \in \mathbb{Z} \backslash\{0\}$ and calculating that

$$
\left|\hat{f}_{k}\right|=\sqrt{2} \cdot \begin{cases}\int_{\mathbb{T}}\left(1-4 x^{2}\right) \mathrm{e}^{-2 \pi \mathrm{i} k x} \mathrm{~d} x=\frac{2 \sqrt{2}}{\pi^{2}|k|^{2}} & \text { for } \mu=\frac{3}{2}, \\ \int_{\mathbb{T}}\left(1-4 x^{2}\right)^{2} \mathrm{e}^{-2 \pi \mathrm{i} k x} \mathrm{~d} x=\frac{24 \sqrt{2}}{\pi^{4}|k|^{4}} & \text { for } \mu=\frac{7}{2}, \\ \int_{\mathbb{T}}\left(1-4 x^{2}\right)^{3} \mathrm{e}^{-2 \pi \mathrm{i} k x} \mathrm{~d} x=\frac{\left.48 \sqrt{2}\left|\pi^{2}\right| k\right|^{2}-15 \mid}{\pi^{6}|k|^{6}} & \text { for } \mu=\frac{11}{2} .\end{cases}
$$

The general $L_{\infty}$-parameter bounds in (5.6) look relatively coarse in comparison to the exact bounds in (5.7). However, generally we can not compute the Fourier coefficients $\hat{f}_{k}$ of a transformed function $f$, which makes the conditions proposed in Theorem 3.4 so powerful as they work independently of the particular choice of $h \in L_{2}(\mathbb{R}, \omega) \cap H^{m}(\mathbb{R})$ at the cost of not yielding the most precise lower parameter bounds.

5.2. Error function transformation. In this section we settle for the constant function $h(\mathbf{y}) \equiv 1$. We could choose $h(\mathbf{y})=\mathrm{e}^{-\|\mathbf{y}\|_{\ell_{2}}^{2}}$ or even the algebraic function $h(\mathbf{y})=\frac{1}{1+\|\mathbf{y}\|_{\ell_{2}}^{2}}$ in (5.1), but they all have the same problem that we are not able to compute their Fourier coefficients $\hat{h}_{\mathbf{k}}$. We proceed in the same way as in the previous section with the algebraic 
transformation. We fix the multivariate version of the Gaussian weight function (3.16), reading as

$$
\omega(\mathbf{y}, \boldsymbol{\mu})=\frac{1}{\pi^{\frac{d}{2}}} \prod_{j=1}^{d} \mathrm{e}^{-\mu_{j}^{2} y_{j}^{2}}
$$

with $\boldsymbol{\mu} \in \mathbb{R}^{d}$, as well as the error function transformation $\psi(\mathbf{x}, \boldsymbol{\eta})=\left(\left(\psi_{j}\left(x_{j}, \eta_{j}\right)\right)_{j=1}^{d}\right)^{\top}$ in the form (3.5) with $\mathbf{x} \in\left(-\frac{1}{2}, \frac{1}{2}\right)^{d}$ and the parameter $\boldsymbol{\eta}=\left(\eta_{1}, \ldots, \eta_{d}\right)^{\top} \in \mathbb{R}^{d}$ and its univariate components given by

$$
\begin{aligned}
\psi_{j}\left(x_{j}, \eta_{j}\right) & =\eta_{j} \operatorname{erf}^{-1}\left(2 x_{j}\right), & \psi_{j}^{\prime}\left(x_{j}, \eta_{j}\right) & =\eta_{j} \sqrt{\pi} \mathrm{e}^{\left(\operatorname{erf}^{-1}\left(2 x_{j}\right)\right)^{2}}, \\
\psi^{-1}\left(y_{j}, \eta_{j}\right) & =\frac{1}{2} \operatorname{erf}\left(\frac{y_{j}}{\eta_{j}}\right), & \varrho\left(y_{j}, \eta_{j}\right) & =\frac{1}{\sqrt{\pi \eta_{j}^{2}}} \mathrm{e}^{-\left(\frac{y_{j}}{\eta_{j}}\right)^{2}} .
\end{aligned}
$$

For $\eta_{j}=1$ we have already stated the definition of $\psi_{j}(\circ, 1)$ in (3.8). For the resulting weighted Hilbert space $L_{2}\left(\mathbb{R}^{d}, \omega(\circ, \boldsymbol{\mu})\right)$, we have a system $\left\{\varphi_{\mathbf{k}}\right\}_{\mathbf{k} \in \mathbb{Z}^{d}}$ of product functions given in (3.36) with univariate components $\left(\varphi_{k_{j}}\right)_{j=1}^{d}$ as in (3.11) of the form

$$
\varphi_{k_{j}}\left(y_{j}, \eta_{j}, \mu_{j}\right)=\frac{1}{\eta_{j}} \mathrm{e}^{\frac{1}{2}\left(\mu_{j}^{2}-\frac{1}{\eta_{j}^{2}}\right) y_{j}^{2}+\pi \mathrm{i} k_{j} \operatorname{erf}\left(\frac{y_{j}}{\eta_{j}}\right)},
$$

which are orthogonal with respect to the weighted scalar product

$$
\left(h_{1}, h_{2}\right)_{L_{2}\left(\mathbb{R}^{d}, \omega(\circ, \boldsymbol{\mu})\right)}=\frac{1}{\pi^{\frac{d}{2}}} \int_{\mathbb{R}^{d}} \prod_{j=1}^{d} \mathrm{e}^{-\mu_{j}^{2} y_{j}^{2}} h_{1}(\mathbf{y}) \overline{h_{2}(\mathbf{y})} \mathrm{d} \mathbf{y} .
$$

The Fourier coefficients $\hat{f}_{\mathbf{k}}$ of an arbitrary function $h \in L_{2}\left(\mathbb{R}^{d}, \omega(\circ, \boldsymbol{\mu})\right)$ are of the form

$$
\begin{aligned}
\hat{h}_{\mathbf{k}}:=\left(h, \varphi_{\mathbf{k}}\right)_{L_{2}\left(\mathbb{R}^{d}, \omega(\circ, \boldsymbol{\mu})\right)} & =\int_{\mathbb{R}^{d}} h(\mathbf{y}) \overline{\varphi_{\mathbf{k}}(\mathbf{y}, \boldsymbol{\eta}, \boldsymbol{\mu})} \omega(\mathbf{y}, \boldsymbol{\mu}) \mathrm{d} \mathbf{y} \\
& =\int_{\mathbb{R}^{d}} h(\mathbf{y}) \prod_{j=1}^{d} \frac{1}{\eta_{j}} \mathrm{e}^{\frac{1}{2}\left(\mu_{j}^{2}-\frac{1}{\eta_{j}^{2}}\right) y_{j}^{2}-\pi \mathrm{i} k_{j} \operatorname{erf}\left(\frac{y_{j}}{\eta_{j}}\right)} \frac{1}{\sqrt{\pi}} \mathrm{e}^{-\mu_{j}^{2} y_{j}^{2}} \mathrm{~d} \mathbf{y} \\
& =\pi^{-\frac{d}{2}} \prod_{j=1}^{d} \frac{1}{\eta_{j}} \int_{\mathbb{R}^{d}} h(\mathbf{y}) \prod_{j=1}^{d} \mathrm{e}^{-\pi \mathrm{i} k_{j} \operatorname{erf}\left(\frac{y_{j}}{\eta_{j}}\right)} \mathrm{e}^{-\frac{1}{2}\left(\mu_{j}^{2}+\frac{1}{\eta_{j}^{2}}\right) y_{j}^{2}} \mathrm{~d} \mathbf{y} .
\end{aligned}
$$

The constant test function $h(y) \equiv 1$ combined with the weight function (5.8) and the transformations (5.9) lead to transformed functions $f$ in the sense of (3.27) of the form

$$
\begin{aligned}
f(\mathbf{x}, \boldsymbol{\eta}, \boldsymbol{\mu}) & =h\left(\psi_{1}\left(x_{1}, \eta_{1}\right), \ldots, \psi_{d}\left(x_{d}, \eta_{d}\right)\right) \prod_{j=1}^{d} \sqrt{\omega_{j}\left(\psi_{j}\left(x_{j}, \eta_{j}\right), \mu_{j}\right) \psi_{j}^{\prime}\left(x_{j}, \eta_{j}\right)} \\
& =\prod_{j=1}^{d} \eta_{j}^{\frac{1}{2}} \mathrm{e}^{\frac{1}{2}\left(1-\mu_{j}^{2} \eta_{j}^{2}\right) \operatorname{erf}^{-1}\left(2 x_{j}\right)^{2}} .
\end{aligned}
$$

In Figure 5.3 we provide a side-by-side comparison of the graphs of these transformed functions with $d=1$ for fixed $\mu^{2}=3$ with varying $1 / 2 \leq \eta^{2} \leq 3$ and for fixed $\eta=1$ with varying $1 \leq \mu^{2} \leq 10$. 

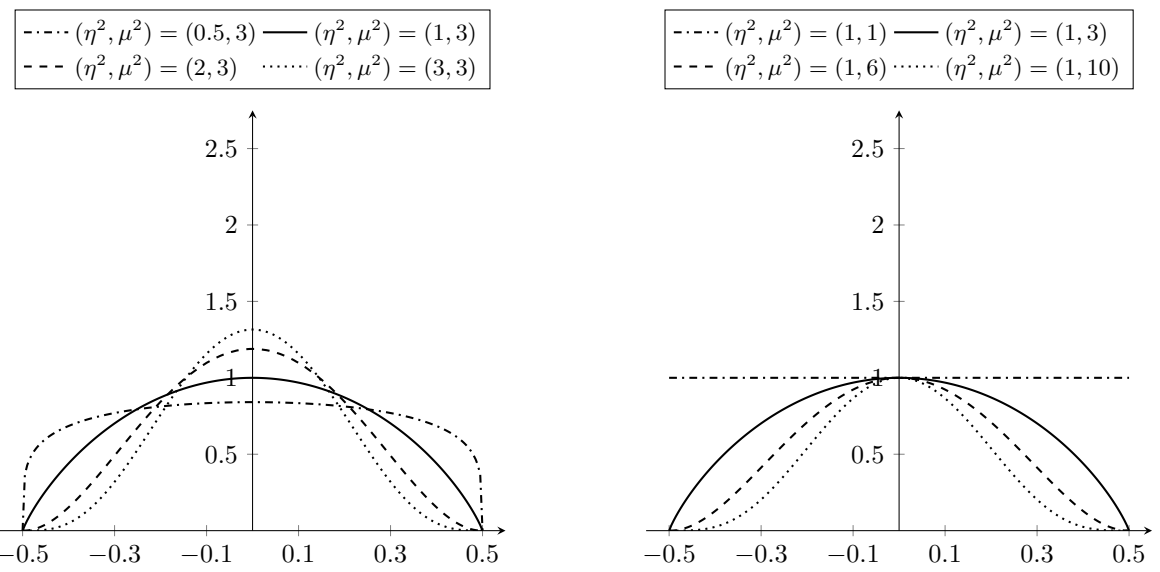

FIG. 5.3. Plots of the univariate transformed function $f$ for various combinations of the parameters $\mu$ and $\eta$ with a Gaussian weight function $\omega$ (5.8) and the error function transformation (5.9). On the left-hand side with fixed $\mu^{2}=3$ and on the right-hand side with fixed $\eta^{2}=1$.

We proceed to determine the values $\boldsymbol{\eta}, \boldsymbol{\mu} \in \mathbb{R}^{d}$ for which $f(\circ, \boldsymbol{\eta}, \boldsymbol{\mu})$ in (5.10) is an element of $H_{\text {mix }}^{m}\left(\mathbb{T}^{d}\right)$ by investigating the conditions (3.28) in Theorem 3.5. First of all, we observe that for $\eta_{1}, \ldots, \eta_{d}>0$, the components $\psi_{1}, \ldots, \psi_{d}$ of the function $\psi(\circ, \boldsymbol{\eta})$ in (5.9) are transformations in the sense of (3.1) by being increasing, continuously differentiable, and invertible functions. Furthermore, for all $\ell=1, \ldots, d$, it is easy to see that its first three derivatives of all $\psi_{j}\left(0, \eta_{j}\right)$ are in fact continuous on $\left(-\frac{1}{2}, \frac{1}{2}\right)$ for $\eta_{j}>0$ and that the first three derivatives of $\varrho_{j}\left(\circ, \eta_{j}\right)$ are in $\mathcal{C}_{0}(\mathbb{R})$ for all non-zero $\eta_{j} \in \mathbb{R}$. Finally, we verify the $L_{\infty}$ conditions (3.28) in Theorem 3.5 for $m=0,1,2,3$. We suppose that for $\ell=1, \ldots, d$ we have $m=m_{\ell}$ and need to inspect if the appearing $L_{\infty}(\mathbb{T})$-norms are finite for all $j_{\ell}=0, \ldots, m$ :

- Let $m=0$. We have

$$
\|\sqrt{\omega(\psi(x, \eta), \mu)}\|_{L_{\infty}(\mathbb{T})}=\pi^{-\frac{1}{4}}\left\|\mathrm{e}^{-\frac{1}{2} \eta^{2} \mu^{2} \operatorname{erf}^{-1}(2 x)^{2}}\right\|_{L_{\infty}(\mathbb{T})}<\infty
$$

for $\eta^{2} \mu^{2} \geq 0$.

- Let $m=1$. We have to verify two conditions. For $j_{\ell}=0$ we have

$$
\begin{aligned}
& \left\|\frac{\partial}{\partial x_{\ell}}\left[\sqrt{\omega_{\ell}\left(\psi_{\ell}\left(x_{\ell}, \eta_{\ell}\right), \mu_{\ell}\right) \psi_{\ell}^{\prime}\left(x_{\ell}, \eta_{\ell}\right)}\right] \psi_{\ell}^{\prime}\left(x_{\ell}, \eta_{\ell}\right)^{-\frac{1}{2}}\right\|_{L_{\infty}(\mathbb{T})} \\
& =\pi^{\frac{1}{4}}\left|\frac{\eta_{\ell}^{2}-\mu_{\ell}^{2}}{\eta_{\ell}^{2}}\right|\left\|\operatorname{erf}^{-1}\left(2 x_{\ell}\right) \mathrm{e}^{-\frac{1}{2}\left(\eta_{\ell}^{2} \mu_{\ell}^{2}-2\right) \operatorname{erf}^{-1}\left(2 x_{\ell}\right)^{2}}\right\|_{L_{\infty}(\mathbb{T})}
\end{aligned}
$$

being finite for $\eta^{2} \mu^{2}>2$. For $j_{\ell}=1$ we have

$$
\begin{aligned}
& \left\|\sqrt{\omega_{\ell}\left(\psi_{\ell}\left(x_{\ell}, \eta_{\ell}\right), \mu_{\ell}\right) \psi_{\ell}^{\prime}\left(x_{\ell}, \eta_{\ell}\right)}\left(\psi_{\ell}^{\prime}\left(x_{\ell}, \eta_{\ell}\right)\right)^{\frac{1}{2}}\right\|_{L_{\infty}(\mathbb{T})} \\
& =\pi^{\frac{1}{4}}\left\|\mathrm{e}^{-\frac{1}{2}\left(\mu_{\ell}^{2} \eta_{\ell}^{2}-2\right)\left(\operatorname{erf}^{-1}\left(2 x_{\ell}\right)^{2}\right.}\right\|_{L_{\infty}(\mathbb{T})},
\end{aligned}
$$

and this is finite if the exponent is negative or zero, which is the case for $\eta^{2} \mu^{2} \geq 2$.

- Let $m=2$. We verify three conditions. For $j_{\ell}=0$

$$
\left\|\frac{\partial^{2}}{\partial x_{\ell}^{2}}\left[\sqrt{\omega_{\ell}\left(\psi_{\ell}\left(x_{\ell}, \eta_{\ell}\right), \mu_{\ell}\right) \psi_{\ell}^{\prime}\left(x_{\ell}, \eta_{\ell}\right)}\right] \psi_{\ell}^{\prime}\left(x_{\ell}, \eta_{\ell}\right)^{-\frac{1}{2}}\right\|_{L_{\infty}(\mathbb{T})}<\infty
$$


for all $\eta_{\ell}^{2} \mu_{\ell}^{2}>4$. For $j_{\ell}=1$

$$
\left\|\frac{\partial}{\partial x_{\ell}}\left[\sqrt{\omega_{\ell}\left(\psi_{\ell}\left(x_{\ell}, \eta_{\ell}\right), \mu_{\ell}\right) \psi_{\ell}^{\prime}\left(x_{\ell}, \eta_{\ell}\right)}\right] \psi_{\ell}^{\prime}\left(x_{\ell}, \eta_{\ell}\right)^{\frac{1}{2}}\right\|_{L_{\infty}(\mathbb{T})}<\infty
$$

for all $\eta_{\ell}^{2} \mu_{\ell}^{2}>4$. For $j_{\ell}=2$

$$
\left\|\sqrt{\omega_{\ell}\left(\psi_{\ell}\left(x_{\ell}, \eta_{\ell}\right), \mu_{\ell}\right) \psi_{\ell}^{\prime}\left(x_{\ell}, \eta_{\ell}\right)}\left(\psi_{\ell}^{\prime}\left(x_{\ell}, \eta_{\ell}\right)\right)^{\frac{5}{2}}\right\|_{L_{\infty}(\mathbb{T})}<\infty
$$

for all $\eta_{\ell}^{2} \mu_{\ell}^{2} \geq 6$.

- For $m=3$ the individual conditions for $k=0,1,2,3$ are finite in case of $\eta_{\ell}^{2} \mu_{\ell}^{2}>6$, $\eta_{\ell}^{2} \mu_{\ell}^{2}>6, \eta_{\ell}^{2} \mu_{\ell}^{2}>8$, and $\eta_{\ell}^{2} \mu_{\ell}^{2} \geq 10$, respectively. Hence, we need $\eta_{\ell}^{2} \mu_{\ell}^{2} \geq 10$ in order to have $f \in H_{\text {mix }}^{3}\left(\mathbb{T}^{d}\right)$.

In total we have calculated that

$$
f \in \begin{cases}H_{\text {mix }}^{1}\left(\mathbb{T}^{d}\right) & \text { for } \eta_{\ell}^{2} \mu_{\ell}^{2} \geq 2 \\ H_{\text {mix }}^{2}\left(\mathbb{T}^{d}\right) & \text { for } \eta_{\ell}^{2} \mu_{\ell}^{2} \geq 6 \\ H_{\text {mix }}^{3}\left(\mathbb{T}^{d}\right) & \text { for } \eta_{\ell}^{2} \mu_{\ell}^{2} \geq 10 .\end{cases}
$$

Contrary to the previous section concerned with the algebraic transformation (5.3), the $L_{2}$-approximation error can not be discussed this time as we are not able to compute the Fourier coefficients

$$
\begin{aligned}
\hat{f}_{\mathbf{k}} & =\int_{\mathbb{T}^{d}} f(\mathbf{x}, \boldsymbol{\eta}, \boldsymbol{\mu}) \mathrm{e}^{-2 \pi \mathrm{ikx}} \mathrm{d} \mathbf{x} \\
& =\int_{\mathbb{T}} h(\psi(\mathbf{x}, \boldsymbol{\eta})) \prod_{j=1}^{d} \eta_{j}^{\frac{1}{2}} \mathrm{e}^{\frac{1}{2}\left(1-\mu_{j}^{2} \eta_{j}^{2}\right) \operatorname{erf}^{-1}\left(2 x_{j}\right)^{2}} \mathrm{e}^{-2 \pi \mathrm{i} k_{j} x_{j}} \mathrm{~d} \mathbf{x}
\end{aligned}
$$

regardless of the chosen $h$. Even for trivial choices of $h$ we are not able to integrate the transformed weight function.

Hence, we only discuss the application of the weighted $L_{\infty}\left(\mathbb{R}^{d}\right)$-approximation error bound from Theorem 3.6 for the dimension $d=2$. With the constant test function given by $h(\mathbf{y})=h\left(y_{1}, y_{2}\right) \equiv 1$ for $d=2$, the weight function (5.8), the transformations (5.9), and the corresponding transformed functions $f$ in (5.10) read as

$$
f(\mathbf{x})=\prod_{j=1}^{2} \sqrt{\omega_{j}\left(\psi_{j}\left(x_{j}, \eta_{j}\right), \mu_{j}\right) \psi_{j}^{\prime}\left(x_{j}, \eta_{j}\right)} .
$$

Let be given $N \geq 8$, the two-dimensional hyperbolic cross $I_{N}^{2}$ as in (1.5), and a reconstructing rank-1 lattice $\Lambda\left(\mathbf{z}, M, I_{N}^{2}\right)$. We have already evaluated the sufficient conditions proposed in Theorem 3.4 yielding lower bounds for $\boldsymbol{\eta}, \boldsymbol{\mu} \geq \mathbf{0}$ such that $f$ is at least of Sobolev smoothness order $m=0,1,2,3$, i.e., $f \in H_{\text {mix }}^{m}\left(\mathbb{T}^{2}\right)$ and thus $f \in \mathcal{H}^{m}\left(\mathbb{T}^{2}\right)$. We fix $\lambda=1$, and for $m \in \mathbb{N}_{0}$ we choose $\boldsymbol{\eta}=\left(\eta_{1}, \eta_{2}\right)^{\top}, \boldsymbol{\mu}=\left(\mu_{1}, \mu_{2}\right)^{\top} \in \mathbb{R}^{2}$ such that $f \in \mathcal{H}^{m+1}\left(\mathbb{T}^{2}\right) \hookrightarrow \mathcal{A}^{m}\left(\mathbb{T}^{2}\right)$. As outlined in (4.4), we expect the discrete approximation error (4.3) to be bounded by

$$
\left\|\mathbf{h}-\mathbf{h}_{\text {approx }}\right\|_{\ell_{\infty}} \leq\left\|f-S_{I_{N}^{d}}^{\Lambda} f\right\|_{L_{\infty}\left(\mathbb{T}^{2}\right)} \lesssim \begin{cases}N^{0} & \text { for } \eta_{j}^{2} \mu_{j}^{2}>0, \\ N^{-1} & \text { for } \eta_{j}^{2} \mu_{j}^{2}>2, \\ N^{-2} & \text { for } \eta_{j}^{2} \mu_{j}^{2} \geq 6, \\ N^{-3} & \text { for } \eta_{j}^{2} \mu_{j}^{2} \geq 10 .\end{cases}
$$




\section{ETNA}

Kent State University and

Johann Radon Institute (RICAM)

$$
\begin{aligned}
& \because(\boldsymbol{\eta}, \boldsymbol{\mu})=(\mathbf{1}, \mathbf{1}) \multimap(\boldsymbol{\eta}, \boldsymbol{\mu})=(\mathbf{1}, \sqrt{\mathbf{3}}) \\
& \because(\boldsymbol{\eta}, \boldsymbol{\mu})=(\mathbf{1}, \sqrt{\mathbf{6}}) \rightarrow(\boldsymbol{\eta}, \boldsymbol{\mu})=(\mathbf{1}, \sqrt{\mathbf{1 0}})
\end{aligned}
$$

$$
h(\mathbf{y})=1
$$

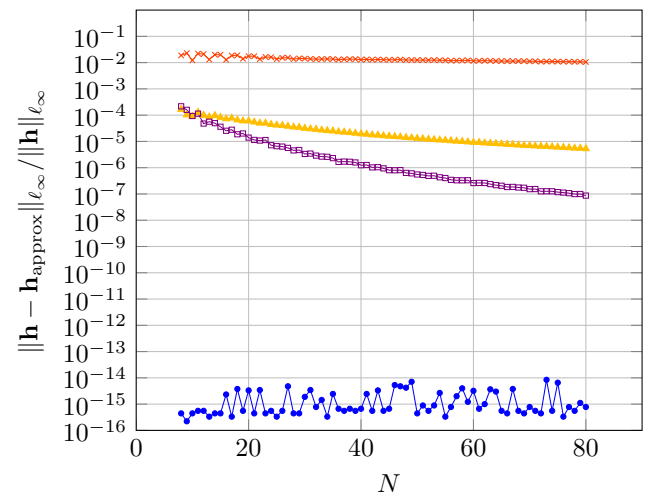

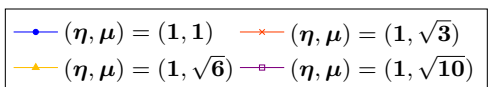

$h(\mathbf{y})=\mathrm{e}^{-y_{1}^{2}-y_{2}^{2}}$

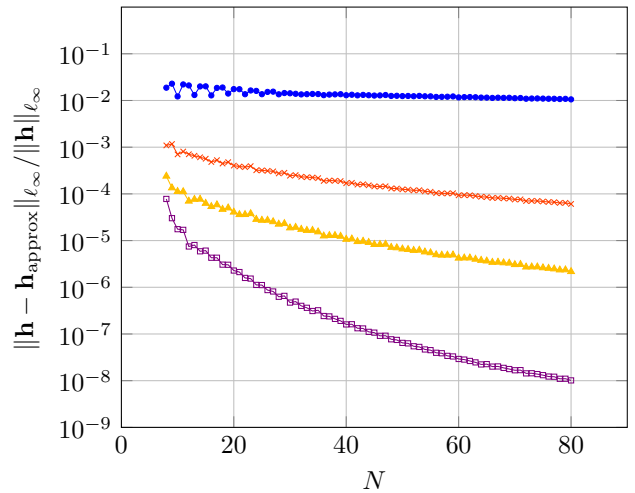

FIG. 5.4. Comparison of the discrete $\ell_{\infty}$-approximation error $\left\|\mathbf{h}-\mathbf{h}_{\text {approx }}\right\|_{\ell_{\infty}} /\|\mathbf{h}\|_{\ell_{\infty}}$ when using the Gaussian weight function $\omega(\circ, \boldsymbol{\mu})$ as in (5.8) and the error function transformation $\psi(\circ, \boldsymbol{\eta})$ as in (5.9) with $\boldsymbol{\mu} \in$ $\{\mathbf{1}, \sqrt{\mathbf{3}}, \sqrt{\mathbf{6}}, \sqrt{\mathbf{1 0}}\}$ and fixed $\boldsymbol{\eta}=\mathbf{1}$.

We actually observe this behavior numerically, as showcased in Figure 5.4, where we display in the left graph the decay of the approximation error of the constant test function $h(\mathbf{y}) \equiv 1$ for $N=8, \ldots, 80$, fixed $\boldsymbol{\eta}=\mathbf{1}$, and $\boldsymbol{\mu} \in\{\mathbf{1}, \sqrt{\mathbf{3}}, \sqrt{\mathbf{6}}, \sqrt{\mathbf{1 0}}\}$. The outlier for $\boldsymbol{\eta}=\boldsymbol{\mu}=\mathbf{1}$ is explained by the fact that the corresponding Fourier coefficients are trivial as these parameters lead to a constant weight function $\omega(\mathbf{y}) \equiv 1$. We repeat this numerical test with the nonconstant test function $h(\mathbf{y})=\mathrm{e}^{-y_{1}^{2}-y_{2}^{2}}$, which is in $L_{2}\left(\mathbb{R}^{2}, \omega(\circ, \boldsymbol{\mu})\right)$ for all $\boldsymbol{\mu} \in \mathbb{R}^{2}$ with $\mu_{1}, \mu_{2}>-2$. Then we have a similar decay of the discrete approximation error as displayed in the right graph of Figure 5.4.

6. Remarks on multiple rank-1 lattices and sparse frequency sets. Now that we are able to construct functions on the torus $\mathbb{T}^{d}$ with a guaranteed minimal Sobolev smoothness degree $m \in \mathbb{N}_{0}$, we adapt the techniques of both multiple rank-1 lattices [12] and sparse FFT algorithms [21]. Usually we consider the algebraic test function in (5.1), which was given by

$$
h(\mathbf{y})=\frac{1}{1+\|\mathbf{y}\|_{\ell_{2}}^{2}}, \quad \mathbf{y} \in \mathbb{R}^{d} .
$$

6.1. Multiple rank-1 lattices. In Lemma 2.1 we recalled that under mild assumptions it is possible to generate a reconstructing rank-1 lattice $\Lambda(\mathbf{z}, M, I)$ with some frequency set $I \subset \mathbb{Z}^{d}$ of finite cardinality $|I|<\infty$ such that

$$
|I| \leq M \leq|I|^{2}
$$

Even though this upper bound is independent of the dimension $d$, the lattice size $M$ is usually close to $|I|^{2}$ and is therefore still quite large. In order to overcome this limitation of the single rank-1 lattice approach, L. Kämmerer suggested the use of multiple rank-1 lattices which are obtained by taking a union of $s$ rank-1 lattices

$$
\Lambda\left(\mathbf{z}_{1}, M_{1}, \ldots, \mathbf{z}_{s}, M_{s}\right):=\bigcup_{j=1, \ldots, s} \Lambda\left(\mathbf{z}_{j}, M_{j}\right)
$$


see $[11,12]$. Then it is possible to determine a reconstructing sampling set for multivariate trigonometric polynomials in $\Pi_{I}$ supported on the given frequency set $I$ with a probability of at least $1-\delta_{s}$, where

$$
\delta_{s}=C_{1} \mathrm{e}^{-C_{2} s}
$$

is an upper bound for the probability that the approach fails and where $C_{1}, C_{2}>0$ are constants. In [11] it was proven that the upper bound for the lattice size improves with high probability to

$$
M \leq C|I| \log |I|
$$

for these particular reconstructing lattices. For the adaptation of this approach in the context of families of transformations $\psi(\circ, \boldsymbol{\eta})$ with $\boldsymbol{\eta} \in \mathbb{R}^{d}$, we analogously consider unions of $s$ transformed rank-1 lattices

$$
\Lambda_{\psi(\circ, \boldsymbol{\eta})}\left(\mathbf{z}_{1}, M_{1}, \ldots, \mathbf{z}_{s}, M_{s}\right):=\bigcup_{j=1, \ldots, s} \Lambda_{\psi(\circ, \boldsymbol{\eta})}\left(\mathbf{z}_{j}, M_{j}\right)
$$

in order to sample the test function $h \in L_{2}\left(\mathbb{R}^{d}, \omega\right)$.

For an example in dimension $d=2$, we consider the test function $h$ in (5.1), the algebraic weight function

$$
\omega(\mathbf{y}, \boldsymbol{\mu}):=\left(\frac{1}{1+y_{1}^{2}}\right)^{\mu_{1}}\left(\frac{1}{1+y_{2}^{2}}\right)^{\mu_{2}}
$$

and the algebraic transformation

$$
\psi(\mathbf{x}, \boldsymbol{\eta})=\left(\frac{2 \eta_{1} x_{1}}{\sqrt{1-4 x_{1}^{2}}}, \frac{2 \eta_{2} x_{2}}{\sqrt{1-4 x_{2}^{2}}}\right)^{\top}
$$

based on their univariate versions in (5.2) and (5.3). We consider the sample data vector

$$
\mathbf{h}=\left(h\left(\mathbf{y}_{j}\right) \sqrt{\frac{\omega\left(\mathbf{y}_{j}, \boldsymbol{\mu}\right)}{\varrho\left(\mathbf{y}_{j}, \boldsymbol{\eta}\right)}}\right)_{j=0}^{M-1}
$$

and the corresponding approximated data vector of the form

$$
\mathbf{h}_{\text {approx }}=\left(\sqrt{\frac{\omega\left(\mathbf{y}_{j}, \boldsymbol{\mu}\right)}{\varrho\left(\mathbf{y}_{j}, \boldsymbol{\eta}\right)}} S_{I_{N}^{d}}^{\Lambda} h\left(\mathbf{y}_{j}\right)\right)_{j=0}^{M-1}
$$

with lattice points $\mathbf{y}_{j}$ in the multiple rank-1 lattice $\Lambda_{\psi(\circ, \eta)}\left(\mathbf{z}_{1}, M_{1}, \ldots, \mathbf{z}_{s}, M_{s}, I\right)$ transformed by the algebraic transformation $\psi(\circ, \boldsymbol{\eta})$ in (6.1). In (5.5) we already discussed that the discretized approximation error defined in (4.3) is bounded above by

$$
\left\|\mathbf{h}-\mathbf{h}_{\text {approx }}\right\|_{\ell_{\infty}} \leq\left\|f-S_{I_{N}^{2}}^{\Lambda} f\right\|_{L_{\infty}\left(\mathbb{T}^{2}\right)} \lesssim N^{-m}
$$

for $\mu_{j} \geq 0$ if $m=0$ and for $\mu_{j}>3 m$ if $m=1,2,3$. Similarly to the results of the numerical test with single rank-1 lattices shown in Figure 5.2, we achieve this behavior of the relative discrete approximation error $\left\|\mathbf{h}-\mathbf{h}_{\text {approx }}\right\|_{\ell_{\infty}} /\|\mathbf{h}\|_{\ell_{\infty}}$ when applying the multiple rank-1 algorithms described in [11,12]. In particular, we adapted [11, Algorithm 6]. For $N=8, \ldots, 80, \boldsymbol{\mu} \in\{\mathbf{0}, \mathbf{4}, \mathbf{1 0}, \mathbf{1 6}\}$, and $\boldsymbol{\eta}=\mathbf{1}$, we initialize this algorithm with the parameters $c=30, n=30$, and $\delta=0.5$ and still have the proposed decay rates of the discrete approximation errors as seen in Figure 6.1. A major advantage of this approach is that we do not have to construct the generating vector $\mathbf{z}$ via component-by-component construction methods, which generally takes quite some time. 


\section{ETNA}

Kent State University and

Johann Radon Institute (RICAM)

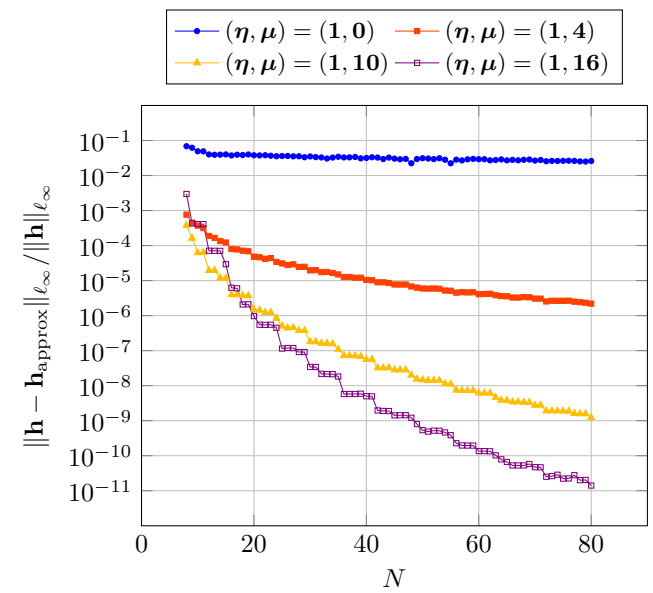

FIG. 6.1. Comparison of the discrete $\ell_{\infty}$-approximation error $\left\|\mathbf{h}-\mathbf{h}_{\text {approx }}\right\|_{\ell_{\infty}} /\|\mathbf{h}\|_{\ell_{\infty}}$ of the test function (5.1) for multiple rank-1 lattices $\Lambda_{\psi(\circ, \boldsymbol{\eta})}\left(\mathbf{z}_{1}, M_{1}, \ldots, \mathbf{z}_{s}, M_{s}\right)$ with the algebraic transformation $\psi(\circ, \boldsymbol{\eta})(5.3)$ and the algebraic weight function $\omega(0, \boldsymbol{\mu})(5.2)$ in their two-dimensional versions with fixed $\boldsymbol{\eta}=\mathbf{1}$ and $\boldsymbol{\mu} \in$ $\{0,4,10,16\}$.

6.2. The construction of sparse frequency sets. Once we set up the transformed function $f$ on the torus of the form (3.17), we can make use of dimension incremental algorithmsthe sparse fast Fourier transforms (sparse FFT) (see [21, 29]) — that reconstruct sparse multivariate trigonometric polynomials with an unknown support in a frequency domain $I \subset \mathbb{Z}^{d}$. Based on a component-by-component construction of rank-1 lattices, the approach of [21, Algorithm 1 and Algorithm 2] describes a dimension incremental construction of a frequency set $I \subset \mathbb{Z}^{d}$ belonging to the non-zero or approximately largest Fourier coefficients. This is achieved by restricting the search space to a full grid $[-N, N]^{d} \cap \mathbb{Z}^{d}$ of refinement $N \in \mathbb{N}$ and by assuming that the cardinality of the support of the multivariate trigonometric polynomial is bounded by a sparsity constraint $s \in \mathbb{N}$. Then we end up with up to $s$ non-zero Fourier coefficients $\hat{f}_{\mathbf{k}}$ of the corresponding test function $f$.

We adapt these algorithms for transformed reconstructing rank-1 lattices $\Lambda_{\psi(\circ, \boldsymbol{\eta})}(\mathbf{z}, M, I)$ by again calculating the relative discretized approximation error $\left\|\mathbf{h}-\mathbf{h}_{\text {approx }}\right\|_{\ell_{\infty}} /\|\mathbf{h}\|_{\ell_{\infty}}$ as in (4.3) with the samples

$$
\mathbf{h}=\left(h\left(\mathbf{y}_{j}\right) \sqrt{\frac{\omega\left(\mathbf{y}_{j}, \boldsymbol{\mu}\right)}{\varrho\left(\mathbf{y}_{j}, \boldsymbol{\eta}\right)}}\right)_{j=0}^{M-1} \quad \text { and } \quad \mathbf{h}_{\text {approx }}=\left(\sqrt{\frac{\omega\left(\mathbf{y}_{j}, \boldsymbol{\mu}\right)}{\varrho\left(\mathbf{y}_{j}, \boldsymbol{\eta}\right)}} S_{I}^{\Lambda} h\left(\mathbf{y}_{j}\right)\right)_{j=0}^{M-1},
$$

but using an unknown frequency set $I$ with cardinality $|I|=s$ that was constructed via a dimensional incremental construction method as outlined above.

6.2.1. An example for the algebraic transformation. We use the algebraic test function (5.1) in combination with the multivariate version of the algebraic weight function (5.2) and the multivariate algebraic transformation based on (5.3) reading as

$$
\omega(\mathbf{y}, \boldsymbol{\mu})=\prod_{j=1}^{d}\left(\frac{1}{1+y_{j}^{2}}\right)^{\mu_{j}}, \quad \psi(\mathbf{x}, \boldsymbol{\eta})=\left(\frac{2 \eta_{1} x_{1}}{\sqrt{1-4 x_{1}^{2}}}, \ldots, \frac{2 \eta_{d} x_{d}}{\sqrt{1-4 x_{d}^{2}}}\right)^{\top}
$$

with $\boldsymbol{\mu}=\mathbf{4}$ and $\boldsymbol{\eta}=\mathbf{1}$. Earlier we have used a similar setup for $d=2$, where we choose a hyperbolic cross $I_{N}^{d}$ as the frequency set. 

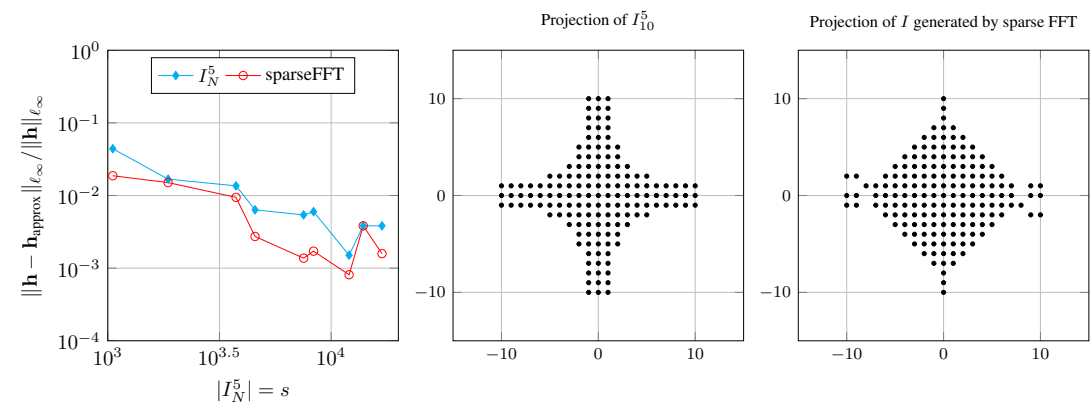

FIG. 6.2. Relative discrete approximation error $\left\|\mathbf{h}-\mathbf{h}_{\text {approx }}\right\|_{\ell_{\infty}} /\|\mathbf{h}\|_{\ell_{\infty}}$ in dimension $d=5$ for the algebraic transformation with the hyperbolic cross $I_{N}^{d}$ with $N=2, \ldots, 10$ compared to the frequency set generated by the sparse FFT algorithm (left). In the center and on the right are the two-dimensional projections of $I_{10}^{5}$ and of the frequency set generated by the sparse FFT algorithm.

Now we let the sparse FFT algorithm [21, Algorithm 2] determine a suitable frequency set $I$. For dimension $d=5$ and for each $N=2,3, \ldots, 10$, we choose the algorithm 'a2r1l' in [29] and use the cardinality of the hyperbolic crosses $I_{N}^{5}$ as the sparsity parameter 'sparsity_s' $=s=\left|I_{N}^{5}\right|$. As expected, the resulting discretized relative approximation errors $\left\|\mathbf{h}-\mathbf{h}_{\text {approx }}\right\|_{\ell_{\infty}} /\|\mathbf{h}\|_{\ell_{\infty}}$ are just as good as the ones where we fixed the hyperbolic cross $I_{N}^{d}$, but the two-dimensional projections of both frequency sets to their first two coordinates differ substantially in size and shape even though they have the same cardinality as seen in Figure 6.2.

6.2.2. An example of the error function transformation and logarithmic transformation. The sparse FFT algorithm is especially interesting for the error function transformation (3.8) and the logarithmic transformation (3.9) because we can not calculate the transformed Fourier coefficients $\hat{h}_{\mathbf{k}}$ given in (3.37). Again we simply let the sparse FFT algorithm [21, Algorithm 2] construct a suitable frequency set $I$ depending on the sparsity $s \in \mathbb{N}$.

We return to dimension $d=2$ and use

$$
h(\mathbf{y})=\mathrm{e}^{-y_{1}^{2}-y_{2}^{2}}
$$

as the test function and consider the constant weight function $\omega(\boldsymbol{y}) \equiv 1$. We again apply two different transformations. The two-dimensional error function transformation

$$
\psi(\boldsymbol{x}, \boldsymbol{\eta})=\left(\eta_{1} \operatorname{erf}^{-1}\left(2 x_{1}\right), \eta_{2} \operatorname{erf}^{-1}\left(2 x_{2}\right)\right)^{\top},
$$

which we consider for $\boldsymbol{\eta}=\mathbf{1}$, is based on its univariate version given in (5.9). The twodimensional logarithmic transformation is also based on its univariate version given in (3.9) and reads as

$$
\psi(\boldsymbol{x}, \boldsymbol{\eta}):=\left(\eta_{1} \log \left(\frac{1+2 x_{1}}{1-2 x_{1}}\right), \eta_{2} \log \left(\frac{1+2 x_{2}}{1-2 x_{2}}\right)\right)^{\top},
$$

which we consider only for $\boldsymbol{\eta}=\mathbf{1}$, too.

At first we fix the refinement $N=20$. Then the full $41 \times 41$-integer grid contains $(2 \cdot 20+1)^{2}=1681$ elements. Again, we initialize the algorithm 'a2r1l' in [29] with the default threshold parameter 'threshold_theta' of $1 \mathrm{e}-12$ and denote the sparsity parameter 'sparsity_s' as $s \in \mathbb{N}$. For the sparsity parameters $s=100$ and $s=500$, the error function 

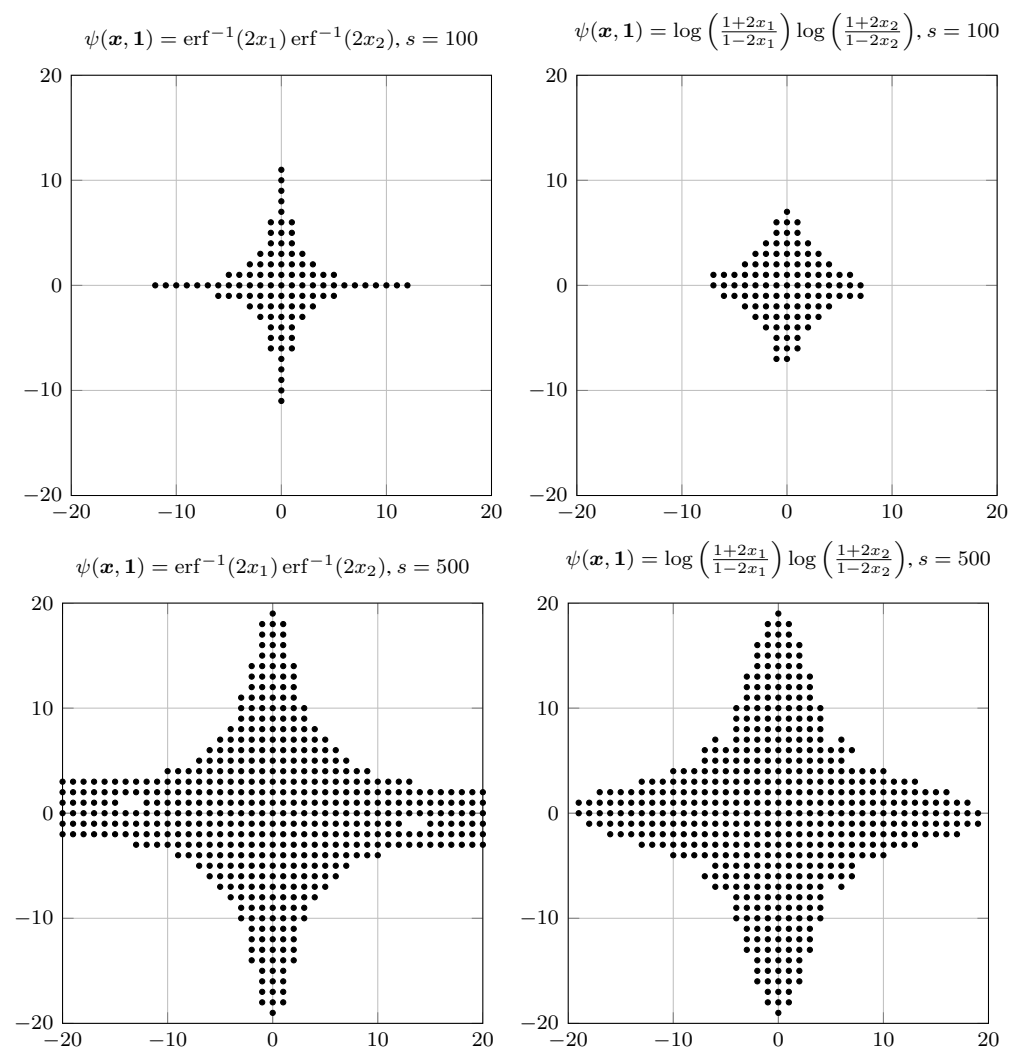

FIG. 6.3. Two-dimensional frequency sets $I_{N}$ with $N=20$ and $s \in\{100,500\}$ for the error function transformation (left column) and the logarithmic transformation (right column).

transformation leads to a frequency set $I_{N}$ that reminds us of a hyperbolic cross, whereas the logarithmic transformation leads to a frequency set that could also resemble an appropriately scaled unit ball $\left\{\mathbf{x} \in \mathbb{Z}^{2}:\left(\left|x_{1}\right|^{p}+\left|x_{2}\right|^{p}\right)^{\frac{1}{p}} \leq N\right\}$ of a two-dimensional sequence space $\ell_{p}$ with $0<p<1$; see Figure 6.3.

Finally, we focus on the two-dimensional error function transformation with $\boldsymbol{\eta}=\mathbf{1}$ and compare the corresponding relative approximation errors $\left\|\mathbf{h}-\mathbf{h}_{\text {approx }}\right\|_{\ell_{\infty}} /\|\mathbf{h}\|_{\ell_{\infty}}$ calculated by the spare FFT algorithm in two different setups. At first we keep the refinement $N=20$ and consider increasing sparsity parameters $s=2, \ldots, 1681$. Hence, for small values of $s$ we have frequency sets that look like hyperbolic crosses, as shown in the left column of Figure 6.3, whose branches along the central axes become thicker as $s$ increases and eventually end up with the full $41 \times 41$-grid. Based on these frequency sets, the relative approximation errors stagnate at a certain point, displayed on the left in Figure 6.4, because the relatively small refinement value forces the algorithm to include frequencies within the $41 \times 41$ grid that do not significantly improve the approximation of $h$. In comparison, we raise the refinement to $N=150$ and let the sparsity parameter $s$ run from 2 to 1681 again so that the resulting frequency sets have the same cardinality as before but keep their hyperbolic cross-like shape, which is shown on the right-hand side of Figure 6.4, where we have the frequency set constructed by the sparse FFT algorithm for $N=150$ and $s=1681$. With these frequency sets we now have steadily decreasing relative approximation errors for increasing sparsity values, as displayed in the center plot of Figure 6.4. 


\section{ETNA}

Kent State University and

Johann Radon Institute (RICAM)

R. NASDALA AND D. POTTS
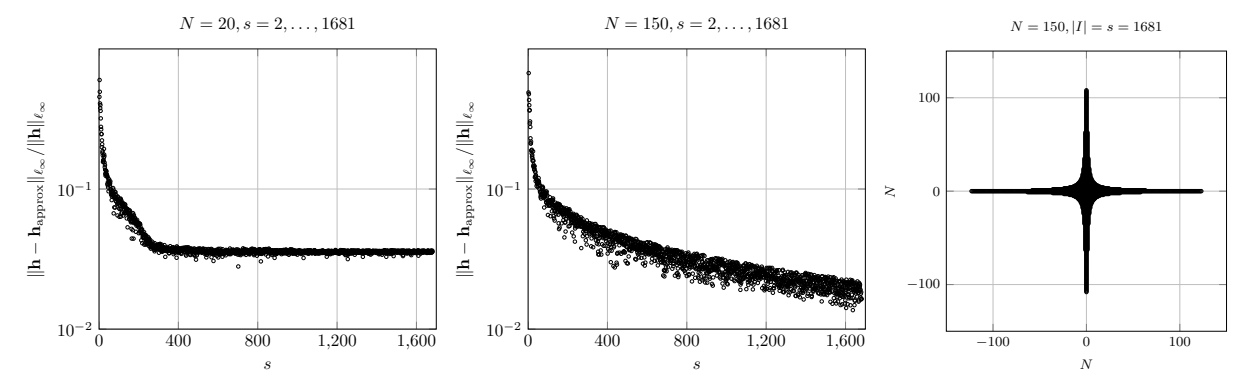

FIG. 6.4. Relative approximation errors $\left\|\mathbf{h}-\mathbf{h}_{\text {approx }}\right\|_{\ell_{\infty}} /\|\mathbf{h}\|_{\ell_{\infty}}$ calculated by the spare FFT algorithm for the error function transformation with $\boldsymbol{\eta}=1, s=2, \ldots, 1681$ and the refinement $N=20$ on the left and $N=150$ in the center. The automatically constructed frequency set I for $N=150, s=1681$ is shown on the right.

6.2.3. An example with the tangent transformation. Finally, we consider a different algebraic test function that is given in product form by

$$
h(\mathbf{y})=\prod_{j=1}^{d} \frac{1}{1+y_{j}^{2}} .
$$

Additionally we consider the constant weight function $\omega(\boldsymbol{y}) \equiv \mathbf{1}$ and the multivariate tangent transformation

$$
\psi(\boldsymbol{x}, \boldsymbol{\eta}):=\left(\eta_{1} \tan \left(\pi x_{1}\right), \ldots, \eta_{d} \tan \left(\pi x_{d}\right)\right)^{\top}
$$

with $\boldsymbol{\eta}=\mathbf{1}$ based on the univariate version defined in (3.7). The resulting transformed function is of the form

$$
f(\boldsymbol{x}, \mathbf{1}, \boldsymbol{\mu}):=\prod_{j=1}^{d} \frac{1}{1+\tan \left(\pi x_{j}\right)^{2}}
$$

This product form extends to the corresponding Fourier coefficients, i.e., $\hat{h}_{\mathbf{k}}=\prod_{j=1}^{d} \hat{h}_{k_{j}}$, and the one-dimensional Fourier coefficients $\hat{h}_{k_{j}}$ are of the form

$$
\hat{h}_{k_{j}}=\int_{-1 / 2}^{1 / 2} \frac{\mathrm{e}^{-2 \pi \mathrm{i} k_{j} x_{j}}}{1+\tan \left(\pi x_{j}\right)^{2}} \mathrm{~d} x_{j}=\int_{-1 / 2}^{1 / 2} \cos \left(\pi x_{j}\right)^{2} \mathrm{e}^{-2 \pi \mathrm{i} k_{j} x_{j}} \mathrm{~d} x_{j}= \begin{cases}\frac{1}{2} & \text { for } k_{j}=0 \\ \frac{1}{4} & \text { for }\left|k_{j}\right|=1 \\ 0 & \text { otherwise }\end{cases}
$$

Hence, over a full grid $[-N, N]^{d} \cap \mathbb{Z}^{d}$ with $(2 N+1)^{d}$ points, there are only $3^{d}$ non-zero multivariate Fourier coefficients $\hat{h}_{\mathbf{k}}$. Again we verify this for the dimension $d=12$ and $N=4$ with the sparse FFT algorithm; see [21, Algorithm 2] and [29]. We initialize this algorithm with the test function $f$ in (6.2), choose the algorithm 'a2r1l', set the sparsity parameter 'sparsity_s' to $10^{6}$ and the threshold parameter 'threshold_theta' to $1 \mathrm{e}-12$. This results in an exact reconstruction as the algorithm indeed only detects the $3^{12}=531441$ out of $(2 \cdot 4+1)^{12} \approx 2.8 \cdot 10^{11}$ possible frequencies corresponding to the 12 -dimensional integer unit cube of radius 1 for which the transformed Fourier coefficients $\hat{h}_{k_{j}}$ are non-zero, as calculated in (6.3). 
7. Conclusion. In this paper we considered functions $h \in L_{2}\left(\mathbb{R}^{d}, \omega(\circ, \boldsymbol{\mu})\right) \cap H_{\text {mix }}^{m}\left(\mathbb{R}^{d}\right)$ with a parameterized weight function $\omega(\circ, \boldsymbol{\mu}): \mathbb{R}^{d} \rightarrow[0, \infty), \boldsymbol{\mu} \in \mathbb{R}^{d}$, and we discussed strategies for transforming them into functions $f$ on the torus $\mathbb{T}^{d}$. A parameterized transformation $\psi(\circ, \boldsymbol{\eta}):\left(-\frac{1}{2}, \frac{1}{2}\right)^{d} \rightarrow \mathbb{R}^{d}$ with parameter $\boldsymbol{\eta} \in \mathbb{R}^{d}$ in combination with the weight function $\omega(\circ, \boldsymbol{\mu})$ allows us to control the degree of smoothness $m \in \mathbb{N}$ of a function $h$ defined on $\mathbb{R}^{d}$, which is preserved under the change of variables $\psi(\circ, \boldsymbol{\eta})$. Hence, the parameters $\boldsymbol{\eta}$ and $\boldsymbol{\mu}$ control which Sobolev space $H_{\text {mix }}^{m}\left(\mathbb{T}^{d}\right)$ the transformed functions $f(\circ, \boldsymbol{\eta}, \boldsymbol{\mu})=h(\psi(\circ, \boldsymbol{\eta})) \sqrt{\omega(\psi(\circ, \boldsymbol{\eta}), \boldsymbol{\mu}) \psi^{\prime}(\circ, \boldsymbol{\eta})}$ belong to. Due to the embedding of the Sobolev space $H_{\text {mix }}^{m}\left(\mathbb{T}^{d}\right)$ into the Wiener algebra $\mathcal{A}\left(\mathbb{T}^{d}\right)$ of functions with absolutely summable Fourier coefficients, we have information on the rate of decay of the Fourier coefficients $\hat{f}_{\mathbf{k}}$ and $\hat{h}_{\mathbf{k}}$ without having to calculate them-which in a lot of cases is not possible. Thus, the essential theoretical $L_{2}$ - and $L_{\infty}$-approximation error bounds on the torus $\mathbb{T}^{d}$ proposed in [30, Theorem 2.30] and [13, Theorem 3.3] can be transferred to $\mathbb{R}^{d}$ by means of the inverse transformation $\psi^{-1}(\circ, \boldsymbol{\eta}): \mathbb{R}^{d} \rightarrow\left(-\frac{1}{2}, \frac{1}{2}\right)^{d}$. Furthermore, only slight modifications are necessary to incorporate such transformations into algorithms based on single reconstructing rank-1 lattices for the evaluation and the reconstruction of transformed multivariate trigonometric polynomials presented in [8, Algorithm 3.1 and 3.2]. Algorithms based on multiple reconstructing rank-1 lattices [12] and sparse fast Fourier transformations [21] can be adjusted, too.

Our numerical tests show that these algorithms are still working within the proposed upper bounds for the approximation error. Additionally, special cases in which we can actually calculate the Fourier coefficients confirm the theoretical parameter bounds for $\boldsymbol{\mu}$ and $\boldsymbol{\eta}$ that are sufficient to achieve a certain degree of Sobolev smoothness under a change of variables. In several examples we apply the adapted multiple rank- 1 lattice methods and adjusted dimension incremental construction methods for sparse frequency sets.

Acknowledgements. The authors thank Lutz Kämmerer, Tino Ullrich, and Toni Volkmer for fruitful discussions during the preparation of this paper. Furthermore, we thank Lutz Kämmerer for the multiple lattice software (see $[9,11]$ ) and Toni Volkmer for the software 'sparseFFTr1l' for computing the sparse fast Fourier transform based on reconstructing rank-1 lattices in a dimension incremental way; see [29]. The first named author gratefully acknowledges the support by the funding of the European Union and the Free State of Saxony (ESF).

\section{REFERENCES}

[1] J. P. Boyd, Chebyshev and Fourier Spectral Methods, 2nd ed., Dover, Mineola, 2001.

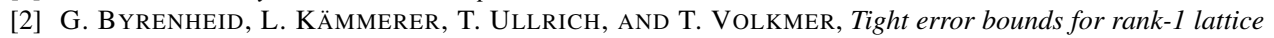
sampling in spaces of hybrid mixed smoothness, Numer. Math., 136 (2017), pp. 993-1034.

[3] B. CHOI, M. IWEN, AND F. KRAHMER, Sparse harmonic transforms: a new class of sublinear-time algorithms for learning functions of many variables, Preprint on arXiv, 2018. https://arxiv.org/abs/1808.04932

[4] R. CoOls, F. Y. KuO, AND D. NuYENS, Constructing lattice rules based on weighted degree of exactness and worst case error, Computing, 87 (2010), pp. 63-89.

[5] R. Cools AND D. NUYENS, Fast algorithms for component-by-component construction of rank-1 lattice rules in shift-invariant reproducing kernel Hilbert spaces, Math. Comp., 75 (2006), pp. 903-920.

[6] J. Dick, F. Y. Kuo, AND I. H. SLOAN, High-dimensional integration: the quasi-Monte Carlo way, Acta Numer., 22 (2013), pp. 133-288.

[7] D. Dung, V. N. TemlYa Kov, AND T. Ullrich, Hyperbolic Cross Approximation, Birkhäuser, Cham, 2018.

[8] L. Kämmerer, High Dimensional Fast Fourier Transform Based on Rank-1 Lattice Sampling, PhD. Thesis, Faculty of Mathematics, TU Chemnitz, Universitätsverlag Chemnitz, Chemnitz, 2014.

[9] - Lattice Based Fast Fourier Transform, Matlab toolbox for the lattice and generated set based FFT, 2014. http://www.tu-chemnitz.de/ lkae/lfft 
[10] — Reconstructing multivariate trigonometric polynomials from samples along rank-1 lattices, in Approximation Theory XIV: San Antonio 2013, G. E. Fasshauer and L. L. Schumaker, eds., Springer, Cham, 2014, pp. 255-271.

[11] Constructing spatial discretizations for sparse multivariate trigonometric polynomials that allow for a fast discrete Fourier transform, Appl. Comput. Harmon. Anal., 47 (2019), pp. 702-729.

[12] _ Multiple rank-1 lattices as sampling schemes for multivariate trigonometric polynomials, J. Fourier Anal. Appl., 24 (2018), pp. 17-44.

[13] L. KäMMERER, D. POTTS, AND T. VOLKMER, Approximation of multivariate periodic functions by trigonometric polynomials based on rank-1 lattice sampling, J. Complexity, 31 (2015), pp. 543-576.

[14] - High-dimensional sparse FFT based on sampling along multiple rank-1 lattices, Preprint on arXiv, 2017. https://arxiv.org/abs/1711.05152

[15] N. M. Korobov, Approximate evaulation of repeated integrals, Dokl. Akad. Nauk SSSR, 124 (1959), pp. 1207-1210.

[16] P. Kritzer, F. Pillichshammer, L. Plaskota, and G. W. Wasilkowski, On efficient weighted integration via a change of variables, Preprint on arXiv, 2018. https://arxiv.org/abs/1812.04259

[17] T. KÜHN, W. Sickel, AND T. ULLRICH, Approximation of mixed order Sobolev functions on the d-torus: asymptotics, preasymptotics, and d-dependence, Constr. Approx., 42 (2015), pp. 353-398.

[18] F. Y. KUO, G. W. WASILKOWSKI, AND B. J. WATERHOUSE, Randomly shifted lattice rules for unbounded integrands, J. Complexity, 22 (2006), pp. 630-651.

[19] H. NiEDERREITER, Quasi-Monte Carlo methods and pseudo-random numbers, Bull. Amer. Math. Soc., 84 (1978), pp. 957-1041, 1978.

[20] G. Plonka, D. Potts, G. STEIdL, And M. Tasche, Numerical Fourier Analysis, Birkhäuser, Cham, 2018.

[21] D. PotTs AND T. VolKmer, Sparse high-dimensional FFT based on rank-1 lattice sampling, Appl. Comput. Harmon. Anal., 41 (2016), pp. 713-748.

[22] H.-J. SChmeISSER AND H. TRIEBel, Topics in Fourier analysis and Function Spaces, Wiley, Chichester, 1987.

[23] J. Shen, T. TANG, And L.-L. Wang, Spectral Methods, Springer, Heidelberg, 2011.

[24] I. H. SLOAN AND S. JoE, Lattice Methods for Multiple Integration, Oxford University Press, New York, 1994.

[25] I. H. SLOAN AND P. J. KACHOYAN, Lattice methods for multiple integration: theory, error analysis and examples, SIAM J. Numer. Anal., 24 (1987), pp. 116-128.

[26] V. N. TEMLYAKOV, Reconstruction of periodic functions of several variables from the values at the nodes of number-theoretic nets, Anal. Math., 12 (1986), pp. 287-305.

[27] - Approximation of Periodic Functions, Nova Science Publishers, Commack, 1993.

[28] T. UllRICH, Smolyak's Algorithm, Sparse Grid Approximation and Periodic Function Spaces with Dominating Mixed Smoothness, PhD. Thesis, Fakultät für Mathematik und Informatik, Friedrich-Schiller-Universität Jena, Jena, 2007.

[29] T. VOLKMER, sparseFFTr1l, Matlab toolbox for computing the sparse fast Fourier transform based on reconstructing rank-1 lattices in a dimension incremental way, 2015. https://www-user.tu-chemnitz.de/ tovo/software.php.en

[30] - Multivariate Approximation and High-Dimensional Sparse FFT Based on Rank-1 Lattice Sampling, $\mathrm{PhD}$. Thesis, Faculty of Mathematics, TU Chemnitz, Universitätsverlag Chemnitz, Chemnitz, 2017.

[31] J. VybIRAL, Function Spaces with Dominating Mixed Smoothness, PhD. Thesis, Fakultät für Mathematik und Informatik, Friedrich-Schiller-Universität Jena, Jena, 2005. 